\title{
Palladium-Catalyzed Mizoroki-Heck Reaction of Nitroarenes and Styrene Derivatives
}

Toshimasa Okita ${ }^{\ddagger}$ Kitty K. Asahara ${ }^{\ddagger}$ Kei Muto, and Junichiro Yamaguchi*

Department of Applied Chemistry, Waseda University, 3-4-1 Ohkubo, Shinjuku, Tokyo 169-8555, Japan.

E-mail: junyamaguchi@waseda.jp

Table of Contents

1. General

2. Synthesis of Nitroarenes $\mathbf{1}$

3. Synthesis of Styrene Derivatives 2

4. Palladium-Catalyzed Denitrative Mizoroki-Heck Reaction

5. Three-Component Coupling by $\mathrm{S}_{\mathrm{N}} \mathrm{Ar} /$ Denitrative Alkenylation

6. Effect of Parameters

7. References

8. $\quad{ }^{1} \mathrm{H}$ and ${ }^{13} \mathrm{C}$ NMR Spectra
S2

S3-S4

S5-S7

S8-S18

S19

S20

S21-S23

S24-S85 


\section{General}

Unless otherwise noted, all reactants or reagents including dry solvents were obtained from commercial suppliers and used as received. Palladium(II) acetylacetonate $\left(\operatorname{Pd}(\mathrm{acac})_{2}\right)$, 1-methyl-4-nitrobenzene (1A), 1-(tert-butyl)-4-nitrobenzene (1B), 1-methoxy-4-nitrobenzene (1C), 1-nitro-4-(trifluoromethyl)benzene (1D), 1-methyl-3-nitrobenzene (1E), 1-methyl-2-nitrobenzene (1F), 2-nitro-1,1'-biphenyl (1G), methyl 4-nitrobenzoate (1H), $N, N$-dimethyl-3-nitroaniline (1J), 1-nitronaphthalene (1K), 6-nitroquinoline (1L), styrene (2a), 1-methyl-4-vinylbenzene (2b), 1-(tert-butyl)-4-vinylbenzene (2c), 1-methoxy-4-vinylbenzene (2d), 1-fluoro-4-vinylbenzene (2i), cyclooctene (2n), tert-butyl acrylate (2p), 1-fluoro-4-nitrobenzene (4) and 3,5-dimethylphenol (5) was $\begin{array}{llll}\text { obtained from } & \text { Tokyo Chemical Industry. }\end{array}$ 2-Dicyclohexylphosphino-3,6-dimethoxy-2',4',6'-triisopropylbiphenyl (BrettPhos) and prop-1-en-2-ylbenzene (2o) was obtained from KANTO Chemical. $\mathrm{Rb}_{2} \mathrm{CO}_{3}$ was obtained from FUJIFILM Wako Pure Chemical Corporation. Unless otherwise noted, all reactions were performed with dry solvents under an atmosphere of $\mathrm{N}_{2}$ in dried glassware using standard vacuum-line techniques. All denitrative Mizoroki-Heck reactions were performed in 20-mL glass vessel equipped with $\mathrm{J}$. Young ${ }^{\circledR}$ O-ring tap and heated (IKA Plate RCT digital) in an oil-bath or a 9-well aluminum reaction block (IKA H 135.103 Block $9 \times 16 \mathrm{ml}$ ) unless otherwise noted. All work-up and purification procedures were carried out with reagent-grade solvents in air.

Analytical thin-layer chromatography (TLC) was performed using Silicagel 70 TLC Plate-Wako $(0.25 \mathrm{~mm})$. The developed chromatogram was analyzed by UV lamp $(254 \mathrm{~nm})$. Flash column chromatography was performed with Biotage Isolera ${ }^{\circledR}$ equipped with Biotage SNAP Ultra columns. Preparative thin-layer chromatography (PTLC) was performed using Wakogel B5-F silica coated plates $(0.75 \mathrm{~mm})$ prepared in our laboratory. Preparative recycling gel permeation chromatography (GPC) was performed with a JAI LaboACE LC-5060 instrument equipped with JAIGEL-2HR columns using $\mathrm{CHCl}_{3}$ as an eluent. Gas chromatography (GC) analysis was conducted on a Shimadzu GC-2010 Plus instrument equipped with a HP-5 column $(30 \mathrm{~m} \times 0.25 \mathrm{~mm}$, Hewlett-Packard $)$ with ${ }^{n}$ decane as an internal standard. High-resolution mass spectra (HRMS) were conducted on Thermo Fisher Scientific ExactivePlus ${ }^{\circledR}$ (DART and ESI). Nuclear magnetic resonance (NMR) spectra were recorded on a JEOL JNM-ECS-400 $\left({ }^{1} \mathrm{H} 400 \mathrm{MHz},{ }^{13} \mathrm{C} 101 \mathrm{MHz}\right)$. Chemical shifts for ${ }^{1} \mathrm{H}$ NMR are expressed in parts per million (ppm) relative to tetramethylsilane ( $\delta 0.00 \mathrm{ppm})$. Chemical shifts for ${ }^{13} \mathrm{C}$ NMR are expressed in ppm relative to $\mathrm{CDCl}_{3}(\delta 77.0 \mathrm{ppm})$. Data are reported as follows: chemical shift, multiplicity $(\mathrm{s}=$ singlet, $\mathrm{d}=$ doublet, $\mathrm{dd}=$ doublet of doublets, $\mathrm{t}=$ triplet, $\mathrm{td}=$ triplet of doublets, $\mathrm{q}=$ quartet, $\mathrm{m}=$ multiplet $)$, coupling constant $(\mathrm{Hz})$, and integration . 


\section{Synthesis of Nitroarenes 1}

\section{2-1. Synthesis of 1 I}<smiles>O=Cc1ccc([N+](=O)[O-])cc1</smiles>
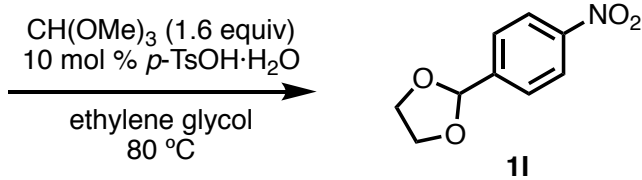

To a solution of 4-nitrobenzaldehyde (378 mg, $2.5 \mathrm{mmol}, 1.0$ equiv) and $p$-toluenesulfonic acid monohydrate $(47.6 \mathrm{mg}, 0.25 \mathrm{mmol}, 10 \mathrm{~mol} \%)$ in ethylene glycol $(4.0 \mathrm{~mL})$ were added trimethyl orthoformate ( $424 \mathrm{mg}, 438 \mu \mathrm{L}, 4.0 \mathrm{mmol}, 1.6$ equiv) at room temperature. Then the mixture was stirring at $80^{\circ} \mathrm{C}$ for $24 \mathrm{~h}$ with monitoring reaction progress with TLC. Upon the completion of the reaction, the mixture was added $\mathrm{NaHCO}_{3}$ aq. and extracted three times with EtOAc. The combined organic layer was dried over $\mathrm{Na}_{2} \mathrm{SO}_{4}$, filtrated, and concentrated in vacuo. The residue was purified by recrystallization (hexane/ $\mathrm{CH}_{2} \mathrm{Cl}_{2}$ ) to afford $\mathbf{1 I}$ as a pale yellow solid (178 $\mathrm{mg}, 36 \%$ yield). ${ }^{1} \mathrm{H}$ NMR $\left(400 \mathrm{MHz}, \mathrm{CDCl}_{3}\right) \delta 8.25(\mathrm{~d}, J=8.8 \mathrm{~Hz}, 2 \mathrm{H}), 7.66(\mathrm{~d}, J=8.8 \mathrm{~Hz}, 2 \mathrm{H}), 5.90(\mathrm{~s}, 1 \mathrm{H}), 4.15-4.05$ (m, $4 \mathrm{H})$. The spectra matched with those of compounds reported in the literature. ${ }^{[1]}$

\section{2-2. Synthesis of $1 M$}<smiles>O=[N+]([O-])c1ccc2[nH]ccc2c1</smiles>
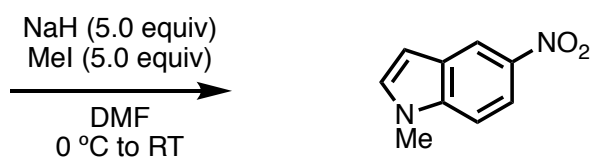

$1 \mathrm{M}$

1-Methyl-5-nitro-1 $H$-indole (1M) was synthesized according to a procedure reported in the literature with a slight modification. ${ }^{[2]}$ To a solution of 5-nitro- $1 H$-indole $(405 \mathrm{mg}, 2.5 \mathrm{mmol}, 1.0$ equiv) in DMF (10 mL) was added sodium hydride (60\% oil dispersion; $500 \mathrm{mg}, 12.5 \mathrm{mmol}, 5.0$ equiv) at $0{ }^{\circ} \mathrm{C}$. After stirring for $30 \mathrm{~min}$ at $0{ }^{\circ} \mathrm{C}$, iodomethane $(1.77 \mathrm{~g}, 778 \mu \mathrm{L}, 12.5 \mathrm{mmol}, 5.0$ equiv) was added dropwise and then the mixture was stirred at room temperature for $12 \mathrm{~h}$ with monitoring reaction progress with TLC. Upon the completion of the reaction, the mixture was added $\mathrm{H}_{2} \mathrm{O}$ and extracted three times with EtOAc. The combined organic layer was dried over $\mathrm{Na}_{2} \mathrm{SO}_{4}$, filtrated, and concentrated in vacuo. The residue was purified by recrystallization (hexane $/ \mathrm{CH}_{2} \mathrm{Cl}_{2}$ ) to afford $\mathbf{1 M}$ as a yellow solid (368 mg, 83\% yield). ${ }^{1} \mathrm{H}$ NMR $\left(400 \mathrm{MHz}, \mathrm{CDCl}_{3}\right) \delta 8.59$ (d, $\left.J=2,4 \mathrm{~Hz}, 1 \mathrm{H}\right), 8.14$ (dd, $J=9.2,2.4 \mathrm{~Hz}, 1 \mathrm{H}), 7.34(\mathrm{~d}, J=9.2 \mathrm{~Hz}, 1 \mathrm{H}), 7.21(\mathrm{~d}, J=3.2 \mathrm{~Hz}, 1 \mathrm{H}), 6.68(\mathrm{~d}, \mathrm{~J}=3.2 \mathrm{~Hz}, 1 \mathrm{H}), 3.87$ $(\mathrm{s}, 3 \mathrm{H})$. The spectra matched with those of compounds reported in the literature. ${ }^{[2]}$

\section{2-3. Synthesis of $1 \mathrm{~N}$}<smiles>COc1cc([N+](=O)[O-])ccc1NS(=O)(=O)[O-]</smiles>

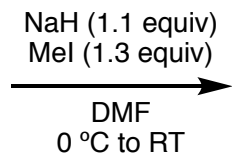<smiles>CO[N+]([O-])(O)c1ccc([N+](=O)[O-])cc1O</smiles> 
To a solution of $N$-(4-nitro-2-phenoxyphenyl)methanesulfonamide (nimesulide: $463 \mathrm{mg}, 1.5 \mathrm{mmol}$, 1.0 equiv) in DMF (3.0 mL) was added sodium hydride (60\% oil dispersion; $66.0 \mathrm{mg}, 1.7 \mathrm{mmol}, 1.1$ equiv) at $0{ }^{\circ} \mathrm{C}$. After stirring for $30 \mathrm{~min}$ at $0{ }^{\circ} \mathrm{C}$, iodomethane (277 mg, $121 \mu \mathrm{L}, 2.0 \mathrm{mmol}, 1.3$ equiv) was added dropwise and then the mixture was stirred at room temperature for $12 \mathrm{~h}$ with monitoring reaction progress with TLC. Upon the completion of the reaction, the mixture was added $\mathrm{NH}_{4} \mathrm{Cl}$ aq. and extracted three times with EtOAc. The combined organic layer was dried over $\mathrm{Na}_{2} \mathrm{SO}_{4}$, filtrated, and concentrated in vacuo. The residue was purified by Isolera ${ }^{\circledR}$ (hexane/EtOAc $=9: 1$ to 3:2) to afford $1 \mathrm{~N}$ as a white solid (480 mg, 99\% yield). ${ }^{1} \mathrm{H} \mathrm{NMR}\left(400 \mathrm{MHz}, \mathrm{CDCl}_{3}\right) \delta 7.92(\mathrm{dd}, J=8.8,2.8 \mathrm{~Hz}, 1 \mathrm{H})$, 7.69-7.58 (m, 2H), 7.47 (t, $J=7.6 \mathrm{~Hz}, 2 \mathrm{H}), 7.35-7.25$ (m, 1H), 7.12 (d, $J=7.6 \mathrm{~Hz}, 2 \mathrm{H}), 3.38(\mathrm{~s}, 3 \mathrm{H})$, $3.03(\mathrm{~s}, 3 \mathrm{H}) ;{ }^{13} \mathrm{C}$ NMR $\left(101 \mathrm{MHz}, \mathrm{CDCl}_{3}\right) \delta 154.7,154.2,147.5,136.6,132.4,130.5,125.6,119.6$, 117.9, 112.5, 38.4, 37.5; HRMS (ESI) $m / z$ calcd for $\mathrm{C}_{14} \mathrm{H}_{14} \mathrm{~N}_{2} \mathrm{O}_{5} \mathrm{SNa}[\mathrm{M}+\mathrm{Na}]^{+}: 345.0516$ found 345.0508 . 


\section{Synthesis of Styrene Derivatives 2}

\section{3-1. General Procedure for the Synthesis of 2}
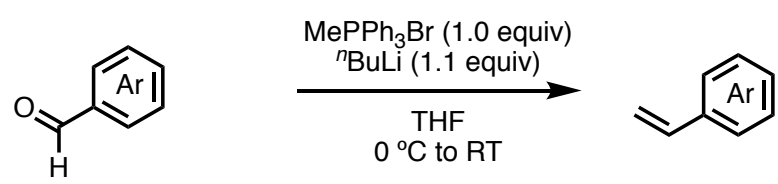

2

To a suspension of methyltriphenylphosphonium bromide (1.0 equiv; azeotroped three times with toluene before use) in THF ( $0.5 \mathrm{M}), n$-butyllithium (1.6 M in hexane, 1.1 equiv) was added under $\mathrm{N}_{2}$ at $0{ }^{\circ} \mathrm{C}$ with stirring. After $30 \mathrm{~min}$, benzaldehydes (1.0 equiv) was added dropwise and then the mixture was stirred at room temperature. After stirring for several hours with monitoring reaction progress with TLC, the mixture was added water and extracted three times with $\mathrm{Et}_{2} \mathrm{O}$. The organic layer was dried over $\mathrm{MgSO}_{4}$, filtrated, and then concentrated in vacuo. Purification by Isolera ${ }^{\circledR}$ to afford 2.

\section{1-Methoxy-3-vinylbenzene (2e)}

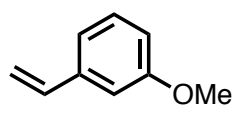

$2 e$

Purification by Isolera ${ }^{\circledR}$ (hexane/ $\mathrm{Et}_{2} \mathrm{O}=99: 1$ to $4: 1$ ) to afford $2 \mathrm{e}$ as a yellow oil (175 $\mathrm{mg}, 44 \%$ yield, $3.0 \mathrm{mmol} \mathrm{scale}) .{ }^{1} \mathrm{H}$ NMR $\left(400 \mathrm{MHz}, \mathrm{CDCl}_{3}\right) \delta 7.24(\mathrm{t}, J=7.6 \mathrm{~Hz}, 1 \mathrm{H}), 7.00(\mathrm{~d}, J=7.6 \mathrm{~Hz}, 1 \mathrm{H})$, 6.98-6.93 (d, $J=2.0,1 \mathrm{H}), 6.82(\mathrm{dd}, J=7.6,2.0 \mathrm{~Hz}, 1 \mathrm{H}), 6.69(\mathrm{dd}, J=17.6,10.8 \mathrm{~Hz}, 1 \mathrm{H}), 5.75(\mathrm{~d}, J=$ $17,6 \mathrm{~Hz}, 1 \mathrm{H}), 5.25(\mathrm{~d}, J=10.8 \mathrm{~Hz}, 1 \mathrm{H}), 3.81(\mathrm{~s}, 3 \mathrm{H})$. The spectra matched with those of compounds reported in the literature. ${ }^{[3]}$

\section{1-Methoxy-2-vinylbenzene (2f)}

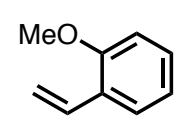

$2 \mathbf{f}$

Purification by Isolera ${ }^{\circledR}$ (hexane/Et $\mathrm{E}_{2} \mathrm{O}=99: 1$ to $4: 1$ ) to afford $\mathbf{2 f}$ as a yellow oil ( $222 \mathrm{mg}, 55 \%$ yield, $3.0 \mathrm{mmol}$ scale). ${ }^{1} \mathrm{H}$ NMR (400 MHz, $\left.\mathrm{CDCl}_{3}\right) \delta 7.47(\mathrm{~d}, J=7.2 \mathrm{~Hz}, 1 \mathrm{H}), 7.26(\mathrm{dd}, J=8.4,8.0 \mathrm{~Hz}, 1 \mathrm{H})$, $7.05(\mathrm{dd}, J=16.4,10.8 \mathrm{~Hz}, 1 \mathrm{H}), 6.93(\mathrm{dd}, J=8.0,7.2 \mathrm{~Hz}, 1 \mathrm{H}), 6.88(\mathrm{~d}, J=8.4 \mathrm{~Hz}, 1 \mathrm{H}), 5.74(\mathrm{~d}, J=$ $16.4 \mathrm{~Hz}, 1 \mathrm{H}), 5.26(\mathrm{~d}, J=10.8 \mathrm{~Hz}, 1 \mathrm{H}), 3.85(\mathrm{~s}, 3 \mathrm{H})$. The spectra matched with those of compounds reported in the literature. ${ }^{[4]}$

\section{$N, N$-Dimethyl-4-vinylaniline (2g)}




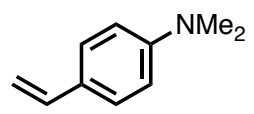

$2 \mathrm{~g}$

Purification by Isolera ${ }^{\circledR}$ (hexane/Et $\mathrm{Et}_{2} \mathrm{O}=99: 1$ to $4: 1$ ) to afford $\mathbf{2 g}$ as a yellow oil (247 $\mathrm{mg}, 56 \%$ yield, $3.0 \mathrm{mmol} \mathrm{scale}) .{ }^{1} \mathrm{H}$ NMR (400 MHz, $\left.\mathrm{CDCl}_{3}\right) \delta 7.31$ (d, $\left.J=8.4 \mathrm{~Hz}, 2 \mathrm{H}\right), 6.68(\mathrm{~d}, J=8.4 \mathrm{~Hz}, 2 \mathrm{H}), 6.63$ (d, $J=18.0,11.2 \mathrm{~Hz}, 1 \mathrm{H}), 5.54(\mathrm{~d}, J=18.0 \mathrm{~Hz}, 1 \mathrm{H}), 5.02$ (d, $J=11.2 \mathrm{~Hz}, 1 \mathrm{H}), 2.96(\mathrm{~s}, 6 \mathrm{H})$. The spectra matched with those of compounds reported in the literature. ${ }^{[5]}$

\section{Trimethyl(4-vinylphenyl)silane (2h)}

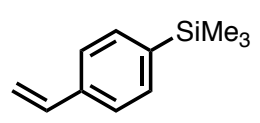

$2 \mathrm{~h}$

Purification by Isolera ${ }^{\circledR}$ (hexane/ $\mathrm{CHCl}_{3}=99: 1$ to $\left.4: 1\right)$ to afford $\mathbf{2 h}$ as a yellow oil $(265 \mathrm{mg}, 50 \%$ yield, $3.0 \mathrm{mmol} \mathrm{scale}) .{ }^{1} \mathrm{H}$ NMR $\left(400 \mathrm{MHz}, \mathrm{CDCl}_{3}\right) \delta 7.49(\mathrm{~d}, J=6.4 \mathrm{~Hz}, 2 \mathrm{H}), 7.39(\mathrm{~d}, J=6.4 \mathrm{~Hz}$, 2H), $6.72(\mathrm{dd}, J=14.4,9.2 \mathrm{~Hz}, 1 \mathrm{H}), 5.77$ (d, $J=14.4 \mathrm{~Hz}, 1 \mathrm{H}), 5.25$ (d, $J=9.2 \mathrm{~Hz}, 1 \mathrm{H}), 0.26$ (s, 9H). The spectra matched with those of compounds reported in the literature. ${ }^{[6]}$

\section{1-Vinylnaphthalene (2k)}

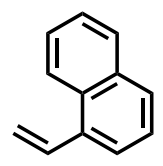

2k

The Wittig reaction was conducted by using 1.2 equiv of methyltriphenylphosphonium bromide and 1.3 equiv of $n$-butyllithium. Purification by Isolera $^{\circledR}$ (hexane) to afford $\mathbf{2 k}$ as a yellow oil (494 $\mathrm{mg}$, 64\% yield, $5.0 \mathrm{mmol}$ scale). ${ }^{1} \mathrm{H}$ NMR $\left(400 \mathrm{MHz}, \mathrm{CDCl}_{3}\right) \delta 8.13(\mathrm{~d}, J=7.6 \mathrm{~Hz}, 1 \mathrm{H}), 7.89-7.84(\mathrm{~m}$, $1 \mathrm{H}), 7.80(\mathrm{~d}, J=7.6 \mathrm{~Hz}, 1 \mathrm{H}), 7.64(\mathrm{~d}, J=7.6 \mathrm{~Hz}, 1 \mathrm{H}), 7.55-7.44(\mathrm{~m}, 4 \mathrm{H}), 5.80(\mathrm{~d}, J=17.6 \mathrm{~Hz}, 1 \mathrm{H})$, $5.48(\mathrm{~d}, J=11.2 \mathrm{~Hz}, 1 \mathrm{H})$. The spectra matched with those of compounds reported in the literature. ${ }^{[7]}$

\section{2-Vinylnaphthalene (2l)}

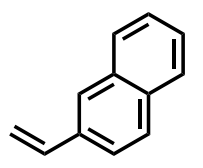

2I

The Wittig reaction was conducted by using 1.2 equiv of methyltriphenylphosphonium bromide and 1.3 equiv of $n$-butyllithium. Purification by Isolera ${ }^{\circledR}$ (hexane) to afford $\mathbf{2 l}$ as a white solid (271 $\mathrm{mg}$, 54\% yield, $3.2 \mathrm{mmol} \mathrm{scale}) .{ }^{1} \mathrm{H}$ NMR (400 MHz, $\left.\mathrm{CDCl}_{3}\right) \delta$ 7.84-7.78 (m, 3H), $7.75(\mathrm{~s}, 1 \mathrm{H}), 7.65$ (dd, 
$J=8.8,2.0 \mathrm{~Hz}, 1 \mathrm{H}), 7.49-7.42(\mathrm{~m}, 2 \mathrm{H}), 6.89(\mathrm{dd}, J=18.0,11.2 \mathrm{~Hz}, 1 \mathrm{H}), 5.88(\mathrm{~d}, J=18.0 \mathrm{~Hz}, 1 \mathrm{H})$, $5.34(\mathrm{~d}, J=11.2 \mathrm{~Hz}, 1 \mathrm{H})$. The spectra matched with those of compounds reported in the literature. ${ }^{[7]}$

\section{4-Vinylisoquinoline (2m)}

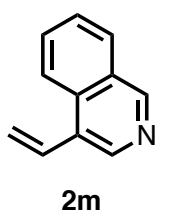

The Wittig reaction was conducted by using 1.1 equiv of methyltriphenylphosphonium bromide and 1.1 equiv of $n$-butyllithium. Purification by Isolera ${ }^{\circledR}$ (hexane/Et $2 \mathrm{O}=8: 1$ to $\mathrm{Et}_{2} \mathrm{O}$ ) to afford $\mathbf{2} \mathbf{m}$ as a yellow oil (52.8 mg, 22\% yield, $1.5 \mathrm{mmol} \mathrm{scale).}{ }^{1} \mathrm{H}$ NMR (400 MHz, $\left.\mathrm{CDCl}_{3}\right) \delta 9.19(\mathrm{~s}, 1 \mathrm{H}), 8.64$ (s, $1 \mathrm{H}), 8.08(\mathrm{~d}, J=8.8 \mathrm{~Hz}, 1 \mathrm{H}), 7.99$ (d, $J=8.4 \mathrm{~Hz}, 1 \mathrm{H}), 7.75$ (dd, $J=8.4,8.0 \mathrm{~Hz}, 1 \mathrm{H}), 7.63$ (dd, $J=8.8$, $8.0 \mathrm{~Hz}, 1 \mathrm{H}), 7.33(\mathrm{dd}, J=17.6,10.8 \mathrm{~Hz}, 1 \mathrm{H}), 5.88(\mathrm{~d}, J=17.6 \mathrm{~Hz}, 1 \mathrm{H}), 5.57$ (d, $J=10.8 \mathrm{~Hz}, 1 \mathrm{H})$. The spectra matched with those of compounds reported in the literature. ${ }^{[8]}$

\section{3-2. Synthesis of $2 j$}
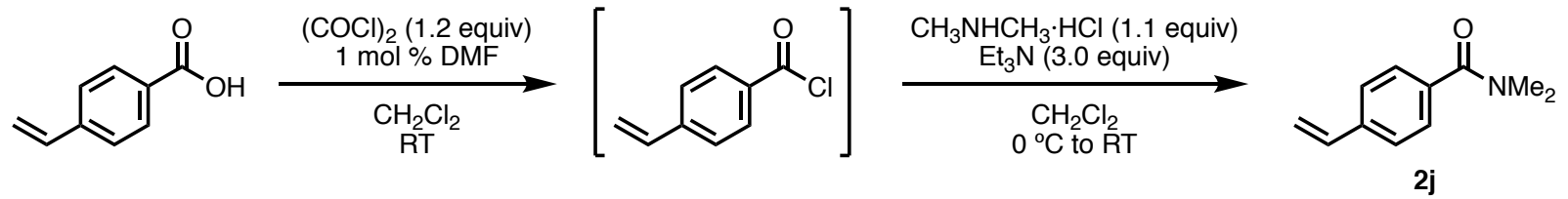

To a solution of 4-vinylbenzoic acid (444 mg, $3.0 \mathrm{mmol}, 1.0$ equiv) in $\mathrm{CH}_{2} \mathrm{Cl}_{2}(6.0 \mathrm{~mL}$ ), oxalyl chloride (457 mg, $308 \mu \mathrm{L}, 3.6 \mathrm{mmol}, 1.2$ equiv) and DMF (3.8 mg, $0.030 \mathrm{mmol}, 1.0 \mathrm{~mol} \%$ ) were added. After stirring for $2 \mathrm{~h}$ at room temperature, the solution was concentrated in vacuo. To the resulted mixture, dimethylamine hydrochloride (269 mg, $3.3 \mathrm{mmol}, 1.1$ equiv) and $\mathrm{CH}_{2} \mathrm{Cl}_{2}(6.0 \mathrm{~mL})$ were added, then triethylamine ( $911 \mathrm{mg}, 1.25 \mathrm{~mL}, 9.0 \mathrm{mmol}, 3.0$ equiv) were added slowly at $0{ }^{\circ} \mathrm{C}$. After stirring the mixture for $17 \mathrm{~h}$ with monitoring the reaction progress by TLC, the reaction mixture was quenched with $\mathrm{H}_{2} \mathrm{O}$ and extracted three times with $\mathrm{CH}_{2} \mathrm{Cl}_{2}$. The combined organic layer was dried over $\mathrm{Na}_{2} \mathrm{SO}_{4}$, filtrated, and concentrated in vacuo. Purification by Isolera ${ }^{\circledR}$ (hexane/EtOAc $=3: 7$ to EtOAc) to afford $N, N$-dimethyl-4-vinylbenzamide (2j) as a white solid (441 $\mathrm{mg}, 84 \%$ yield). ${ }^{1} \mathrm{H}$ NMR $\left(400 \mathrm{MHz}, \mathrm{CDCl}_{3}\right) \delta 7.47-7.37(\mathrm{~m}, 4 \mathrm{H}), 6.73(\mathrm{dd}, J=18.0,10.4 \mathrm{~Hz}, 1 \mathrm{H}), 5.80(\mathrm{~d}, J=18.0 \mathrm{~Hz}, 1 \mathrm{H})$, $5.32(\mathrm{~d}, J=10.4 \mathrm{~Hz}, 1 \mathrm{H}) 3.11(\mathrm{~s}, 3 \mathrm{H}), 3.00(\mathrm{~s}, 3 \mathrm{H})$. The spectra matched with those of compounds reported in the literature. ${ }^{[9]}$ 


\section{Palladium-Catalyzed Denitrative Mizoroki-Heck Reaction}

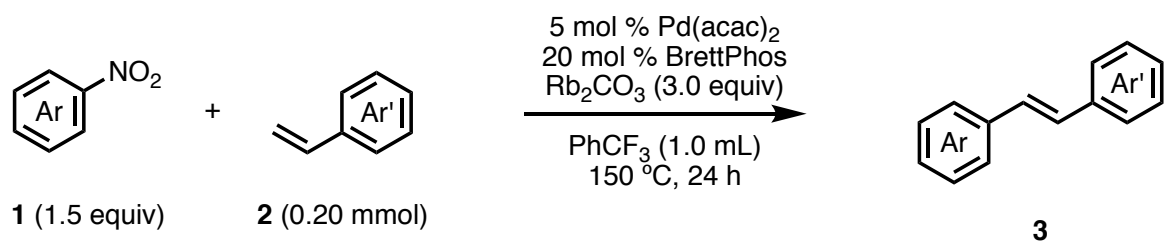

\section{General Procedure}

A 20-mL glass vessel equipped with J. Young ${ }^{\circledR}$ O-ring tap containing a magnetic stirring bar and $\mathrm{Rb}_{2} \mathrm{CO}_{3}$ (139 mg, $0.60 \mathrm{mmol}, 3.0$ equiv) was dried with a heat-gun in vacuo and filled with $\mathrm{N}_{2}$ after cooling to room temperature. To this vessel were added nitroarene 1 ( $0.30 \mathrm{mmol}, 1.5$ equiv), 2-dicyclohexylphosphino-3,6-dimethoxy-2',4',6'-triisopropylbiphenyl (BrettPhos: $21.5 \mathrm{mg}, 0.040$ mmol, $20 \mathrm{~mol} \%)$, and $\mathrm{Pd}(\mathrm{acac})_{2}(3.1 \mathrm{mg}, 0.010 \mathrm{mmol}, 5 \mathrm{~mol} \%)$. The vessel was placed under vacuum and refilled $\mathrm{N}_{2}$ gas three times, and then added alkene $2(0.20 \mathrm{mmol}, 1.0$ equiv) and (trifluoromethyl)benzene $\left(\mathrm{PhCF}_{3}: 1.0 \mathrm{~mL}\right)$. The vessel was sealed with O-ring tap and then heated at $150{ }^{\circ} \mathrm{C}$ for $24 \mathrm{~h}$ with stirring. After cooling the reaction mixture to room temperature, the mixture was passed through a short silica-gel/Celite ${ }^{\circledR}(1: 1)$ pad with EtOAc or $\mathrm{CHCl}_{3} / \mathrm{MeOH}=19: 1$ as an eluent, and then concentrated in vacuo. The residue was purified by PTLC, Isolera ${ }^{\circledR}$, or GPC to afford the corresponding alkene 3 .

\section{Photographic Guideline}

1. A J-Young ${ }^{\circledR}$ glass vessel containing a magnetic stirring bar (Photo A).

2. Drying up the vessel containing $\mathrm{Rb}_{2} \mathrm{CO}_{3}$ with a heat-gun for 2-3 min in vacuo (Photo B).

3. All materials were added (Photo $\mathrm{C}$ ).

4. The vessel was heated with stirring (in a 9-well aluminum block).

Color changing (Photo D: 2 min; E: 10 min; F: 1 h; G: 12 h; H: 24 h).

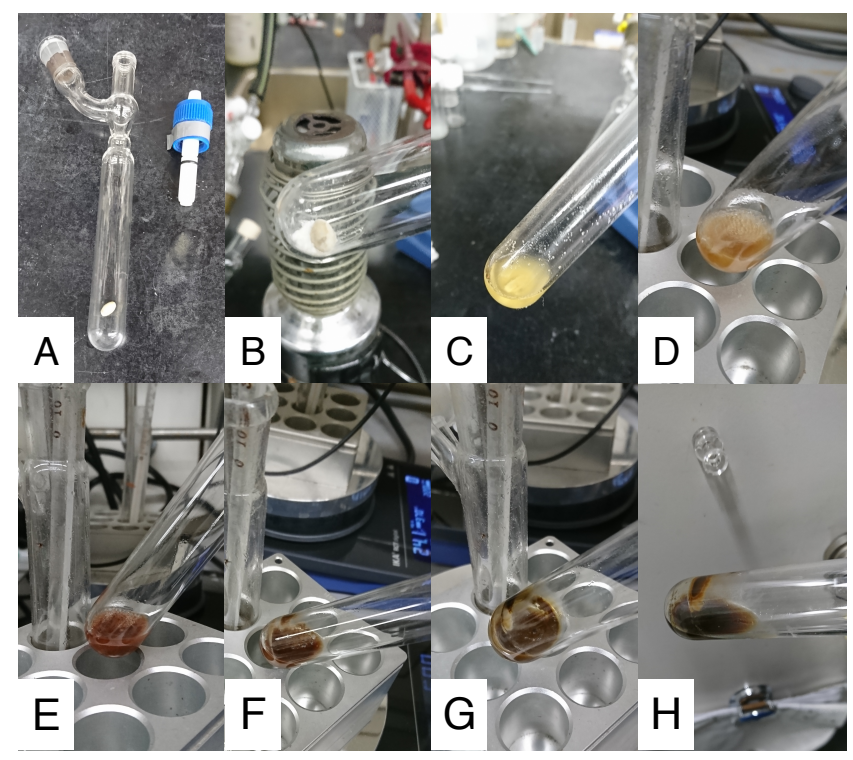

Figure S1 


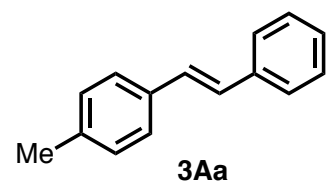

\section{(E)-1-Methyl-4-styrylbenzene (3Aa) ${ }^{[10]}$}

Purification by PTLC (hexane/ $\left.\mathrm{CHCl}_{3}=99: 1\right)$ afforded 3Aa as a white solid (28.1 $\mathrm{mg}, 72 \%$ yield). ${ }^{1} \mathrm{H}$ NMR (400 MHz, $\left.\mathrm{CDCl}_{3}\right) \delta 7.50(\mathrm{dd}, J=7.2,1.6 \mathrm{~Hz}, 2 \mathrm{H}), 7.41(\mathrm{~d}, J=7.6 \mathrm{~Hz}, 2 \mathrm{H}), 7.34(\mathrm{t}, J=7.2$ $\mathrm{Hz}, 2 \mathrm{H}), 7.23(\mathrm{td}, J=7.2,1.6 \mathrm{~Hz}, 1 \mathrm{H}), 7.16(\mathrm{~d}, J=7.6 \mathrm{~Hz}, 2 \mathrm{H}), 7.09$ (d, $J=16.4 \mathrm{~Hz}, 1 \mathrm{H}), 7.04$ (d, $J=$ $16.4 \mathrm{~Hz}, 1 \mathrm{H}), 2.35$ (s, 3H); ${ }^{13} \mathrm{C} \mathrm{NMR}\left(101 \mathrm{MHz}, \mathrm{CDCl}_{3}\right) \delta 137.5,134.5,129.4,128.62,128.59,127.7$, 127.4, 126.40, 126.37, 21.2 (one peak is missing due to overlapping); HRMS (DART) $\mathrm{m} / \mathrm{z}$ calcd for $\mathrm{C}_{15} \mathrm{H}_{15}[\mathrm{M}+\mathrm{H}]^{+}: 195.1168$ found 195.1169 .

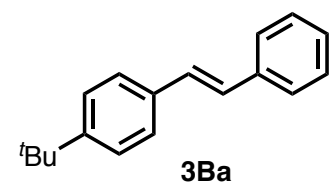

\section{(E)-1-(tert-Butyl)-4-styrylbenzene (3Ba) ${ }^{[11]}$}

Purification by PTLC (hexane/ $\mathrm{CH}_{2} \mathrm{Cl}_{2}=19: 1$, three times) afforded 3Ba as a white solid $(29.1 \mathrm{mg}$, $62 \%$ yield). ${ }^{1} \mathrm{H}$ NMR (400 MHz, $\left.\mathrm{CDCl}_{3}\right) \delta 7.50(\mathrm{~d}, J=7.6 \mathrm{~Hz}, 2 \mathrm{H}), 7.45(\mathrm{dd}, J=8.8,1.2 \mathrm{~Hz}, 2 \mathrm{H})$, $7.38(\mathrm{~d}, J=7.6 \mathrm{~Hz}, 2 \mathrm{H}), 7.35$ (dd, $J=8.8,8.4 \mathrm{~Hz}, 2 \mathrm{H}), 7.23(\mathrm{td}, J=8.4,1.2 \mathrm{~Hz}, 1 \mathrm{H}), 7.11(\mathrm{~d}, J=16.4$ $\mathrm{Hz}, 1 \mathrm{H}), 7.06(\mathrm{~d}, J=16.4 \mathrm{~Hz}, 1 \mathrm{H}), 1.33(\mathrm{~s}, 9 \mathrm{H}) ;{ }^{13} \mathrm{C} \mathrm{NMR}\left(101 \mathrm{MHz}, \mathrm{CDCl}_{3}\right) \delta 150.7,137.5,134.5$, 128.6, 128.4, 127.9, 127.4, 126.4, 126.2, 125.6, 34.6, 31.3; HRMS (DART) $m / z$ calcd for $\mathrm{C}_{18} \mathrm{H}_{20}[\mathrm{M}]^{+}$: 236.1560 found 236.1557 .

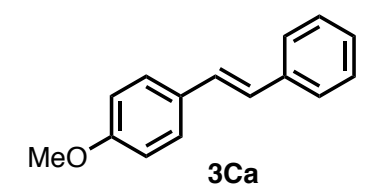

\section{(E)-1-Methoxy-4-styrylbenzene (3Ca) ${ }^{[12]}$}

0.20 mmol scale: Purification by PTLC (hexane/ $\mathrm{CH}_{2} \mathrm{Cl}_{2}=9: 1$, then 5:1) afforded $3 \mathrm{Ca}$ as a white solid (33.1 mg, 79\% yield).

$1.0 \mathrm{mmol}$ scale: Purification by Isolera ${ }^{\circledR}$ (hexane/ $\mathrm{CH}_{2} \mathrm{Cl}_{2}=14: 1$ to $2: 3$ ) afforded $3 \mathrm{Ca}$ as a white solid (167.1 mg, 79\% yield). ${ }^{1} \mathrm{H}$ NMR (400 MHz, $\left.\mathrm{CDCl}_{3}\right) \delta 7.49(\mathrm{dd}, J=7.6,1.6 \mathrm{~Hz}, 2 \mathrm{H}), 7.46$ (d, $J=$ $8.4 \mathrm{~Hz}, 2 \mathrm{H}), 7.35$ (t, $J=8.4 \mathrm{~Hz}, 2 \mathrm{H}), 7.23(\mathrm{td}, J=8.4,1.6 \mathrm{~Hz}, 1 \mathrm{H}), 7.07$ (d, $J=16.4 \mathrm{~Hz}, 1 \mathrm{H}), 6.97$ (d, $J=16.4 \mathrm{~Hz}, 1 \mathrm{H}), 6.90(\mathrm{~d}, J=8.4 \mathrm{~Hz}, 2 \mathrm{H}), 3.83(\mathrm{~s}, 3 \mathrm{H}) ;{ }^{13} \mathrm{C} \mathrm{NMR}\left(101 \mathrm{MHz}, \mathrm{CDCl}_{3}\right) \delta 159.2,137.6$, $130.1,128.6,128.1,127.7,127.2,126.5,126.2,114.1,55.3$; HRMS (DART) $m / z$ calcd for $\mathrm{C}_{15} \mathrm{H}_{15} \mathrm{O}$ $[\mathrm{M}+\mathrm{H}]^{+}: 211.1117$ found 211.1117 . 


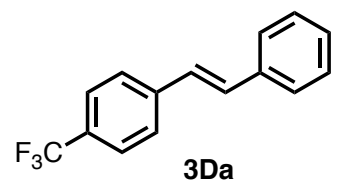

(E)-1-Styryl-4-(trifluoromethyl)benzene (3Da) ${ }^{[13]}$

Purification by PTLC (hexane/ $\mathrm{CHCl}_{3}=99: 1$ ) afforded 3Da as a white solid ( $28.3 \mathrm{mg}, 57 \%$ yield). ${ }^{1} \mathrm{H}$ NMR (400 MHz, $\left.\mathrm{CDCl}_{3}\right) \delta 7.63-7.59(\mathrm{~m}, 4 \mathrm{H}), 7.54$ (d, $\left.J=7.6 \mathrm{~Hz}, 2 \mathrm{H}\right), 7.39$ (d, $\left.J=7.6 \mathrm{~Hz}, 2 \mathrm{H}\right)$, 7.33-7.28 (m, 1H), $7.20(\mathrm{~d}, J=16.4 \mathrm{~Hz}, 1 \mathrm{H}), 7.12(\mathrm{~d}, J=16.4 \mathrm{~Hz}, 1 \mathrm{H}) ;{ }^{13} \mathrm{C}$ NMR $\left(101 \mathrm{MHz}, \mathrm{CDCl}_{3}\right)$ $\delta 140.7,136.6,131.1,129.2\left(\mathrm{q}, J_{\mathrm{C}-\mathrm{F}}=33 \mathrm{~Hz}\right), 128.8,128.3,127.1,126.8,126.5,125.6\left(\mathrm{q}, J_{\mathrm{C}-\mathrm{F}}=3.8\right.$ $\mathrm{Hz}), 124.2\left(\mathrm{q}, J_{\mathrm{C}-\mathrm{F}}=276 \mathrm{~Hz}\right.$ ); HRMS (DART) $\mathrm{m} / z$ calcd for $\mathrm{C}_{15} \mathrm{H}_{11} \mathrm{~F}_{3}[\mathrm{M}]^{+}: 248.0807$ found 248.0801 .

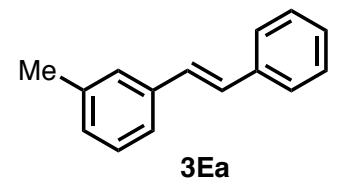

\section{(E)-1-Methyl-3-styrylbenzene (3Ea) $)^{[14]}$}

Purification by PTLC (hexane/ $\mathrm{CH}_{2} \mathrm{Cl}_{2}=9: 1$, then 19:1) afforded 3Ea as a white solid (32.2 $\mathrm{mg}$, $83 \%$ yield). ${ }^{1} \mathrm{H}$ NMR $\left(400 \mathrm{MHz}, \mathrm{CDCl}_{3}\right) \delta 7.51(\mathrm{~d}, J=8.0 \mathrm{~Hz}, 2 \mathrm{H}), 7.39-7.30(\mathrm{~m}, 4 \mathrm{H}), 7.25$ (dd, $J=$ 8.0, 7.6 Hz, 2H), 7.14-7.04 (m, 3H), 2.37 (s, 3H); ${ }^{13} \mathrm{C} \mathrm{NMR} \mathrm{(101} \mathrm{MHz,} \mathrm{CDCl}_{3}$ ) $\delta 138.2,137.4,137.2$, $128.7,128.64,128.55,128.4,127.5,127.2,126.4,123.7,21.4$ (one peak is missing due to overlapping); HRMS (DART) $m / z$ calcd for $\mathrm{C}_{15} \mathrm{H}_{15}[\mathrm{M}+\mathrm{H}]^{+}: 195.1168$ found 195.1168 .

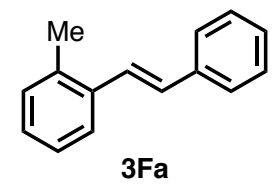

\section{(E)-1-Methyl-2-styrylbenzene $(3 \mathrm{Fa})^{[14]}$}

Purification by PTLC (hexane/ $\mathrm{CH}_{2} \mathrm{Cl}_{2}=19: 1$ ) afforded 3Fa as a white solid (30.4 $\mathrm{mg}, 78 \%$ yield). ${ }^{1} \mathrm{H}$ NMR $\left(400 \mathrm{MHz}, \mathrm{CDCl}_{3}\right) \delta 7.59(\mathrm{~d}, J=7.6 \mathrm{~Hz}, 1 \mathrm{H}), 7.52(\mathrm{~d}, J=8.0 \mathrm{~Hz}, 2 \mathrm{H}), 7.39-7.29(\mathrm{~m}, 3 \mathrm{H})$, $7.26(\mathrm{t}, J=7.6 \mathrm{~Hz}, 1 \mathrm{H}), 7.23-7.14(\mathrm{~m}, 3 \mathrm{H}), 6.99$ (d, $J=16.0 \mathrm{~Hz}, 1 \mathrm{H}), 2.42(\mathrm{~s}, 3 \mathrm{H}) ;{ }^{13} \mathrm{C}$ NMR $(101$ $\left.\mathrm{MHz}, \mathrm{CDCl}_{3}\right) \delta 137.6,136.4,135.8,130.4,130.0,128.7,127.6,127.5,126.53,126.49,126.2,125.3$, 19.9; HRMS (DART) $m / z$ calcd for $\mathrm{C}_{15} \mathrm{H}_{14}[\mathrm{M}]^{+}: 194.1090$ found 194.1089.

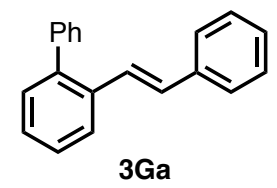

(E)-2-Styryl-1,1'-biphenyl (3Ga) ${ }^{[15]}$

Purification by PTLC (hexane) afforded 3Ga as a colorless oil (15.4 mg, 30\% yield). ${ }^{1} \mathrm{H}$ NMR (400 $\left.\mathrm{MHz}, \mathrm{CDCl}_{3}\right) \delta 7.76(\mathrm{~d}, J=7.6 \mathrm{~Hz}, 1 \mathrm{H}), 7.47-7.33(\mathrm{~m}, 10 \mathrm{H}), 7.30(\mathrm{t}, J=7.6 \mathrm{~Hz}, 2 \mathrm{H}), 7.22(\mathrm{t}, J=7.6$ $\mathrm{Hz}, 1 \mathrm{H}), 7.12(\mathrm{~d}, J=16.4 \mathrm{~Hz}, 1 \mathrm{H}), 7.04(\mathrm{~d}, J=16.4 \mathrm{~Hz}, 1 \mathrm{H}) ;{ }^{13} \mathrm{C} \mathrm{NMR}\left(101 \mathrm{MHz}, \mathrm{CDCl}_{3}\right) \delta 141.1$, 
$140.8,137.5,135.4,130.3,129.9,129.4,128.6,128.1,128.0,127.8,127.53,127.46,127.1,126.5$, 125.8; HRMS (DART) $m / z$ calcd for $\mathrm{C}_{20} \mathrm{H}_{20} \mathrm{~N}\left[\mathrm{M}+\mathrm{NH}_{4}\right]^{+}: 274.1590$ found 274.1587.

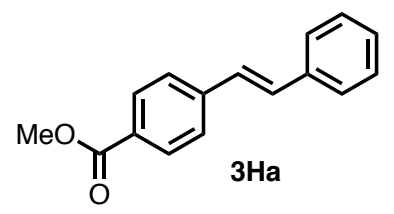

\section{Methyl (E)-4-styrylbenzoate (3Ha) ${ }^{[14]}$}

Purification by Isolera ${ }^{\circledR}$ (hexane/EtOAc $=24: 1$ to $\left.2: 1\right)$ afforded 3Ha as a white solid $(24.4 \mathrm{mg}, 51 \%$ yield). ${ }^{1} \mathrm{H}$ NMR (400 MHz, $\left.\mathrm{CDCl}_{3}\right) \delta 8.02(\mathrm{~d}, J=8.4 \mathrm{~Hz}, 2 \mathrm{H}), 7.56(\mathrm{~d}, J=8.4 \mathrm{~Hz}, 2 \mathrm{H}), 7.53(\mathrm{~d}, J=$ $8.0 \mathrm{~Hz}, 2 \mathrm{H}), 7.38(\mathrm{t}, J=8.0 \mathrm{~Hz}, 2 \mathrm{H}), 7.32-7.17(\mathrm{~m}, 2 \mathrm{H}), 7.12(\mathrm{~d}, J=16.4 \mathrm{~Hz}, 1 \mathrm{H}), 3.92(\mathrm{~s}, 3 \mathrm{H}) ;{ }^{13} \mathrm{C}$ NMR $\left(101 \mathrm{MHz}, \mathrm{CDCl}_{3}\right) \delta 166.8,141.8,136.7,131.2,130.0,128.85,128.75,128.2,127.5,126.8$, 126.3, 52.1; HRMS (DART) $m / z$ calcd for $\mathrm{C}_{16} \mathrm{H}_{15} \mathrm{O}_{2}[\mathrm{M}+\mathrm{H}]^{+}: 239.1067$ found 239.1063 .

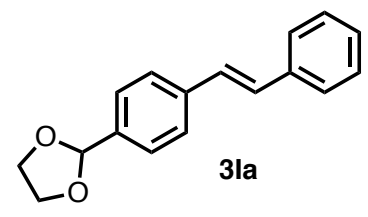

\section{(E)-2-(4-Styrylphenyl)-1,3-dioxolane (3Ia)}

Purification by PTLC (hexane/EtOAc = 5:1) afforded 3Ia as a white solid (21.6 mg, 43\% yield). ${ }^{1} \mathrm{H}$ $\operatorname{NMR}\left(400 \mathrm{MHz}, \mathrm{CDCl}_{3}\right) \delta 7.53(\mathrm{~d}, J=8.4 \mathrm{~Hz}, 2 \mathrm{H}), 7.51(\mathrm{dd}, J=7.6,1.6 \mathrm{~Hz}, 2 \mathrm{H}), 7.47(\mathrm{~d}, J=8.4 \mathrm{~Hz}$, 2H), $7.36(\mathrm{t}, J=7.6 \mathrm{~Hz}, 2 \mathrm{H}), 7.26(\mathrm{td}, J=7.6,1.6 \mathrm{~Hz}, 1 \mathrm{H}), 7.15-7.07(\mathrm{~m}, 2 \mathrm{H}), 5.82(\mathrm{~s}, 1 \mathrm{H}), 4.19-3.99$ (m, 4H); ${ }^{13} \mathrm{C}$ NMR (101 MHz, $\left.\mathrm{CDCl}_{3}\right) \delta 138.2,137.1,137.0,129.2,128.7,128.1,127.7,126.8,126.5$, 126.4, 103.5, 65.3; HRMS (DART) $m / z$ calcd for $\mathrm{C}_{17} \mathrm{H}_{17} \mathrm{O}_{2}[\mathrm{M}+\mathrm{H}]^{+}: 253.1223$ found 253.1219.

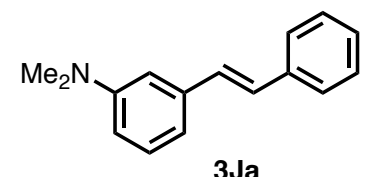

(E)-N,N-Dimethyl-3-styrylaniline (3Ja) ${ }^{[16]}$

Purification by PTLC (hexane/EtOAc $=9: 1)$ afforded 3Ja as a pale yellow solid $(28.4 \mathrm{mg}, 64 \%$ yield). ${ }^{1} \mathrm{H} \mathrm{NMR}\left(400 \mathrm{MHz}, \mathrm{CDCl}_{3}\right) \delta 7.51$ (d, $\left.J=7.6 \mathrm{~Hz}, 2 \mathrm{H}\right), 7.34(\mathrm{t}, J=7.6 \mathrm{~Hz}, 2 \mathrm{H}), 7.27-7.19$ (m, 2H), 7.12-7.06 (m, 2H), 6.92 (d, $J=7.6 \mathrm{~Hz}, 1 \mathrm{H}), 6.85$ (s, 1H), 6.67 (d, $J=7.6 \mathrm{~Hz}, 1 \mathrm{H}), 2.98$ (s, 6H); ${ }^{13} \mathrm{C}$ NMR $\left(101 \mathrm{MHz}, \mathrm{CDCl}_{3}\right) \delta 150.8,138.0,137.5,129.6,129.3,128.6,128.2,127.4,126.4,115.0$, 112.2, 110.8, 40.7; HRMS (DART) $m / z$ calcd for $\mathrm{C}_{16} \mathrm{H}_{18} \mathrm{~N}[\mathrm{M}+\mathrm{H}]^{+}: 224.1434$ found 224.1430. 


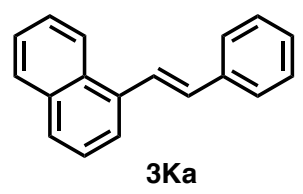

(E)-1-Styrylnaphthalene $(3 \mathrm{Ka})^{[7]}$

Purification by GPC afforded $3 \mathrm{Ka}$ as a yellow solid (28.5 mg, 62\% yield). ${ }^{1} \mathrm{H}$ NMR (400 MHz, $\left.\mathrm{CDCl}_{3}\right) \delta 8.23(\mathrm{~d}, J=8.4 \mathrm{~Hz}, 1 \mathrm{H}), 7.95-7.86(\mathrm{~m}, 2 \mathrm{H}), 7.82(\mathrm{~d}, J=8.0 \mathrm{~Hz}, 1 \mathrm{H}), 7.76(\mathrm{~d}, J=7.6 \mathrm{~Hz}$, $1 \mathrm{H}), 7.62(\mathrm{~d}, J=8.0 \mathrm{~Hz}, 2 \mathrm{H}), 7.59-7.48(\mathrm{~m}, 3 \mathrm{H}), 7.41(\mathrm{t}, J=8.0 \mathrm{~Hz}, 2 \mathrm{H}), 7.34-7.28(\mathrm{~m}, 1 \mathrm{H}), 7.16(\mathrm{~d}$, $J=16.0 \mathrm{~Hz}, 1 \mathrm{H}) ;{ }^{13} \mathrm{C}$ NMR $\left(101 \mathrm{MHz}, \mathrm{CDCl}_{3}\right) \delta 137.6,135.0,133.7,131.7,131.3,128.7,128.6$, 128.0, 127.8, 126.7, 126.1, 125.81, 125.75, 125.7, 123.7, 123.6; HRMS (DART) $m / z$ calcd for $\mathrm{C}_{18} \mathrm{H}_{15}$ $[\mathrm{M}+\mathrm{H}]^{+}: 231.1168$ found 231.1164 .

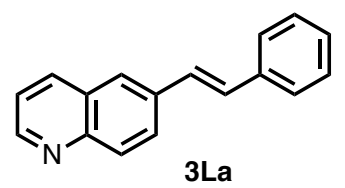

\section{(E)-6-Styrylquinoline (3La) ${ }^{[17]}$}

Purification by PTLC (hexane/EtOAc $=2: 1$, twice) afforded 3La as a yellow solid $(30.3 \mathrm{mg}, 66 \%$ yield). ${ }^{1} \mathrm{H}$ NMR (400 MHz, $\left.\mathrm{CDCl}_{3}\right) \delta 8.87(\mathrm{dd}, J=4.0,1.6 \mathrm{~Hz}, 1 \mathrm{H}), 8.15(\mathrm{dd}, J=7.6,1.2 \mathrm{~Hz}, 1 \mathrm{H})$, $8.09(\mathrm{~d}, J=7.6 \mathrm{~Hz}, 1 \mathrm{H}), 7.99(\mathrm{dd}, J=8.8,1.6 \mathrm{~Hz}, 1 \mathrm{H}), 7.83(\mathrm{~d}, J=1.2 \mathrm{~Hz}, 1 \mathrm{H}), 7.58$ (dd, $J=7.2,1.6$ $\mathrm{Hz}, 2 \mathrm{H}), 7.43-7.36$ (m, 3H), 7.33-7.27 (m, 3H); ${ }^{13} \mathrm{C} \mathrm{NMR}\left(101 \mathrm{MHz}, \mathrm{CDCl}_{3}\right) \delta$ 150.1, 148.0, 136.9, $135.9,135.5,130.1,129.7,128.7,128.5,128.0,127.8,127.2,126.6,125.9,121.5$; HRMS (ESI) $\mathrm{m} / \mathrm{z}$ calcd for $\mathrm{C}_{17} \mathrm{H}_{14} \mathrm{~N}[\mathrm{M}+\mathrm{H}]^{+}: 232.1119$ found 232.1121 .

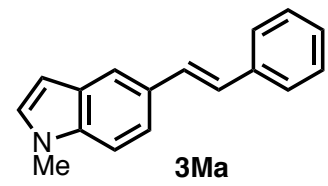

(E)-1-Methyl-5-styryl-1H-indole (3Ma) ${ }^{[18]}$

Purification by PTLC (hexane/EtOAc $=4: 1)$ afforded 3Ma as a pale orange solid $(19.6 \mathrm{mg}, 42 \%$ yield). ${ }^{1} \mathrm{H}$ NMR (400 MHz, $\left.\mathrm{CDCl}_{3}\right) \delta 7.73(\mathrm{~d}, J=1.2 \mathrm{~Hz}, 1 \mathrm{H}), 7.52(\mathrm{~d}, J=7.2 \mathrm{~Hz}, 2 \mathrm{H}), 7.46$ (dd, $J=$ 8.8, $1.2 \mathrm{~Hz}, 1 \mathrm{H}), 7.35$ (t, $J=7.2 \mathrm{~Hz}, 2 \mathrm{H}), 7.30-7.20(\mathrm{~m}, 3 \mathrm{H}), 7.07$ (d, $J=16.0 \mathrm{~Hz}, 1 \mathrm{H}), 7.03$ (d, $J=$ $3.2 \mathrm{~Hz}, 1 \mathrm{H}), 6.48(\mathrm{~d}, J=3.2 \mathrm{~Hz}, 1 \mathrm{H}), 3.77(\mathrm{~s}, 3 \mathrm{H}) ;{ }^{13} \mathrm{C} \mathrm{NMR}\left(101 \mathrm{MHz}, \mathrm{CDCl}_{3}\right) \delta 138.0,136.5,130.1$, 129.4, 128.9, 128.7, 128.6, 126.9, 126.1, 125.8, 120.1, 119.7, 109.4, 101.3, 32.9; HRMS (DART) $\mathrm{m} / \mathrm{z}$ calcd for $\mathrm{C}_{17} \mathrm{H}_{16} \mathrm{~N}[\mathrm{M}+\mathrm{H}]^{+}: 234.1277$ found 234.1272 . 


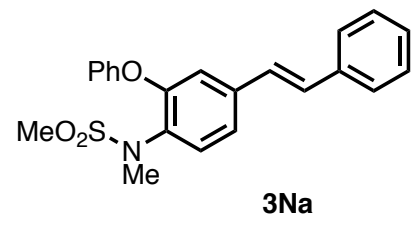

(E)-N-Methyl- $N$-(2-phenoxy-4-styrylphenyl)methanesulfonamide (3Na)

Purification by PTLC (hexane/EtOAc $=4: 1$, then 3:1) afforded $\mathbf{3 N a}$ as a yellow solid $(23.7 \mathrm{mg}$, $31 \%$ yield). ${ }^{1} \mathrm{H}$ NMR (400 MHz, $\left.\mathrm{CDCl}_{3}\right) \delta 7.50-7.39(\mathrm{~m}, 5 \mathrm{H}), 7.33(\mathrm{t}, J=7.6 \mathrm{~Hz}, 2 \mathrm{H}), 7.30-7.25(\mathrm{~m}$, $2 \mathrm{H}), 7.23-7.18(\mathrm{~m}, 1 \mathrm{H}), 7.08(\mathrm{~d}, J=8.0 \mathrm{~Hz}, 2 \mathrm{H}), 7.02-6.92(\mathrm{~m}, 3 \mathrm{H}), 3.32(\mathrm{~s}, 3 \mathrm{H}), 2.96(\mathrm{~s}, 3 \mathrm{H}) ;{ }^{13} \mathrm{C}$ NMR $\left(101 \mathrm{MHz}, \mathrm{CDCl}_{3}\right) \delta 155.9,154.1,139.1,136.6,132.7,130.4,130.2,128.7,128.1,127.0,126.6$, 124.3, 121.7, 118.9, 116.5, 38.0 (two peaks are missing due to overlapping); HRMS (ESI) $\mathrm{m} / \mathrm{z}$ calcd for $\mathrm{C}_{22} \mathrm{H}_{21} \mathrm{NO}_{3} \mathrm{SNa}[\mathrm{M}+\mathrm{Na}]^{+}: 402.1134$ found 402.1127 .

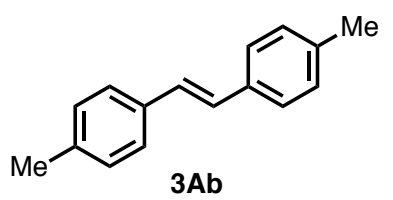

(E)-1,2-di-p-Tolylethene (3Ab) ${ }^{[19]}$

Purification by PTLC (hexane/ $\mathrm{CH}_{2} \mathrm{Cl}_{2}=19: 1$, four times) afforded $\mathbf{3 A b}$ as a white solid (26.4 mg, $63 \%$ yield). ${ }^{1} \mathrm{H}$ NMR (400 MHz, $\left.\mathrm{CDCl}_{3}\right) \delta 7.40(\mathrm{~d}, J=8.4 \mathrm{~Hz}, 4 \mathrm{H}), 7.15$ (d, $\left.J=8.4 \mathrm{~Hz}, 4 \mathrm{H}\right), 7.03$ (s, $2 \mathrm{H}), 2.35(\mathrm{~s}, 6 \mathrm{H}) ;{ }^{13} \mathrm{C}$ NMR $\left(101 \mathrm{MHz}, \mathrm{CDCl}_{3}\right) \delta 137.2,134.7,129.3,127.6,126.3,21.2$; HRMS (DART) $m / z$ calcd for $\mathrm{C}_{16} \mathrm{H}_{17}[\mathrm{M}+\mathrm{H}]^{+}: 209.1325$ found 209.1323 .

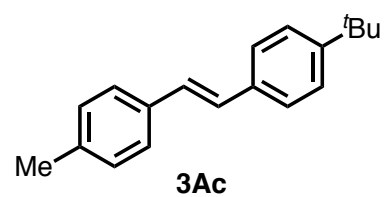

(E)-1-(tert-Butyl)-4-(4-methylstyryl)benzene $(3 \mathrm{Ac})^{[20]}$

Purification by PTLC (hexane/ $\mathrm{CHCl}_{3}=99: 1$ ) afforded 3Ac as a white solid (40.0 $\mathrm{mg}, 80 \%$ yield).

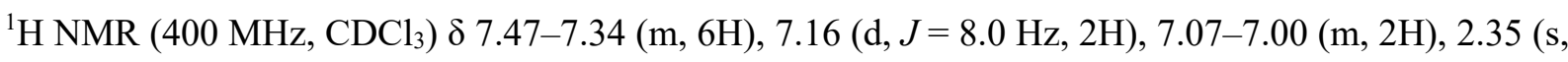
$3 \mathrm{H}), 1.33(\mathrm{~s}, 9 \mathrm{H}) ;{ }^{13} \mathrm{C}$ NMR $\left(101 \mathrm{MHz}, \mathrm{CDCl}_{3}\right) \delta 150.5,137.3,134.7,129.3,127.8,127.5,126.3$, 126.1, 125.6, 34.6, 31.3, 21.2 (one peak is missing due to overlapping); HRMS (DART) $\mathrm{m} / \mathrm{z}$ calcd for $\mathrm{C}_{19} \mathrm{H}_{23}[\mathrm{M}+\mathrm{H}]^{+}: 251.1794$ found 251.1789 . 


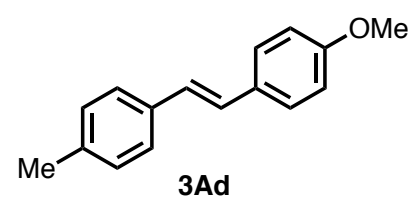

(E)-1-Methoxy-4-(4-methylstyryl) benzene (3Ad) ${ }^{[21]}$

Purification by PTLC (hexane/ $\mathrm{CH}_{2} \mathrm{Cl}_{2}=2: 1$ ) afforded 3Ad as a yellow solid (39.8 $\mathrm{mg}, 89 \%$ yield). ${ }^{1} \mathrm{H}$ NMR (400 MHz, $\left.\mathrm{CDCl}_{3}\right) \delta 7.44(\mathrm{~d}, J=8.8 \mathrm{~Hz}, 2 \mathrm{H}), 7.39$ (d, $\left.J=8.0 \mathrm{~Hz}, 2 \mathrm{H}\right), 7.15$ (d, $J=8.8 \mathrm{~Hz}$, 2H), 7.02 (d, $J=16.4 \mathrm{~Hz}, 1 \mathrm{H}), 6.95$ (d, $J=16.4 \mathrm{~Hz}, 1 \mathrm{H}), 6.89$ (d, $J=8.0 \mathrm{~Hz}, 2 \mathrm{H}), 3.83$ (s, 3H), 2.35 $(\mathrm{s}, 3 \mathrm{H}) ;{ }^{13} \mathrm{C}$ NMR $\left(101 \mathrm{MHz}, \mathrm{CDCl}_{3}\right) \delta 159.1,137.0,134.8,130.3,129.3,127.5,127.2,126.5,126.1$, 114.0, 55.2, 21.2; HRMS (DART) $m / z$ calcd for $\mathrm{C}_{16} \mathrm{H}_{17} \mathrm{O}[\mathrm{M}+\mathrm{H}]^{+}: 225.1274$ found 225.1269.

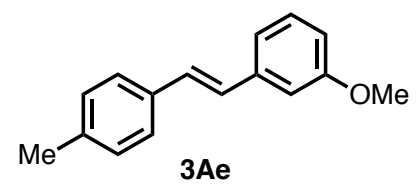

\section{(E)-1-Methoxy-3-(4-methylstyryl)benzene (3Ae $)^{[22]}$}

Purification by PTLC (hexane/ $\left.\mathrm{CH}_{2} \mathrm{Cl}_{2}=1: 1\right)$ afforded 3Ae as a yellow solid (27.0 mg, $60 \%$ yield). ${ }^{1} \mathrm{H}$ NMR (400 MHz, $\left.\mathrm{CDCl}_{3}\right) \delta 7.41(\mathrm{~d}, J=8.0 \mathrm{~Hz}, 2 \mathrm{H}), 7.27$ (t, $\left.J=7.6 \mathrm{~Hz}, 1 \mathrm{H}\right), 7.17$ (d, $J=8.0 \mathrm{~Hz}$, 2H), $7.11(\mathrm{~d}, J=7.6 \mathrm{~Hz}, 1 \mathrm{H}), 7.08-7.03(\mathrm{~m}, 3 \mathrm{H}), 6.81(\mathrm{dd}, J=7.6,2.8 \mathrm{~Hz}, 1 \mathrm{H}), 3.85(\mathrm{~s}, 3 \mathrm{H}), 2.36$ (s, $3 \mathrm{H}) ;{ }^{13} \mathrm{C}$ NMR $\left(101 \mathrm{MHz}, \mathrm{CDCl}_{3}\right) \delta 159.8,138.9,137.6,134.4,129.6,129.4,128.9,127.5,126.4$, 119.1, 113.1, 111.6, 55.2, 21.2; HRMS (DART) $\mathrm{m} / z$ calcd for $\mathrm{C}_{16} \mathrm{H}_{17} \mathrm{O}[\mathrm{M}+\mathrm{H}]^{+}: 225.1274$ found 225.1269 .

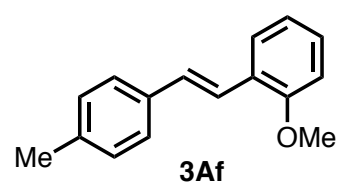

(E)-1-Methoxy-2-(4-methylstyryl)benzene (3Af $)^{[23]}$

Purification by PTLC (hexane/ $\mathrm{CH}_{2} \mathrm{Cl}_{2}=2: 1$ ) afforded 3Af as a white solid (32.1 $\mathrm{mg}, 72 \%$ yield). ${ }^{1} \mathrm{H}$ NMR $\left(400 \mathrm{MHz}, \mathrm{CDCl}_{3}\right) \delta 7.59(\mathrm{~d}, J=7.6 \mathrm{~Hz}, 1 \mathrm{H}), 7.44(\mathrm{~d}, J=16.4 \mathrm{~Hz}, 1 \mathrm{H}), 7.43(\mathrm{~d}, J=7.6 \mathrm{~Hz}$, 2H), $7.22(\mathrm{dd}, J=8.0,7.6 \mathrm{~Hz}, 1 \mathrm{H}), 7.15(\mathrm{~d}, J=7.6 \mathrm{~Hz}, 2 \mathrm{H}), 7.08$ (d, $J=16.4 \mathrm{~Hz}, 1 \mathrm{H}), 6.96$ (t, $J=7.6$ $\mathrm{Hz}, 1 \mathrm{H}), 6.88(\mathrm{~d}, J=8.0 \mathrm{~Hz}, 1 \mathrm{H}), 3.87(\mathrm{~s}, 3 \mathrm{H}), 2.35(\mathrm{~s}, 3 \mathrm{H}) ;{ }^{13} \mathrm{C} \mathrm{NMR}\left(101 \mathrm{MHz}, \mathrm{CDCl}_{3}\right) \delta 156.7$, $137.2,135.1,129.3,129.0,128.4,126.5,126.4,126.2,122.3,120.7,110.8,55.4,21.2$; HRMS (DART) $\mathrm{m} / \mathrm{z}$ calcd for $\mathrm{C}_{16} \mathrm{H}_{17} \mathrm{O}[\mathrm{M}+\mathrm{H}]^{+}: 225.1274$ found 225.1268 . 


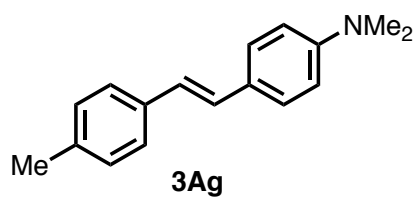

(E)-N,N-Dimethyl-4-(4-methylstyryl)aniline $(3 \mathrm{Ag})^{[24]}$

Purification by PTLC (hexane/ $\mathrm{CH}_{2} \mathrm{Cl}_{2}=2: 1$ ) afforded $\mathbf{3 A g}$ as a white solid (34.6 $\mathrm{mg}, 73 \%$ yield). ${ }^{1} \mathrm{H}$ NMR (400 MHz, $\left.\mathrm{CDCl}_{3}\right) \delta 7.40(\mathrm{~d}, J=8.0 \mathrm{~Hz}, 2 \mathrm{H}), 7.38(\mathrm{~d}, J=8.0 \mathrm{~Hz}, 2 \mathrm{H}), 7.14(\mathrm{~d}, J=8.0 \mathrm{~Hz}$, 2H), $7.00(\mathrm{~d}, J=16.0 \mathrm{~Hz}, 1 \mathrm{H}), 6.89$ (d, $J=16.0 \mathrm{~Hz}, 1 \mathrm{H}), 6.72$ (d, $J=8.0 \mathrm{~Hz}, 2 \mathrm{H}), 2.98(\mathrm{~s}, 6 \mathrm{H}), 2.34$ $(\mathrm{s}, 3 \mathrm{H}) ;{ }^{13} \mathrm{C}$ NMR $\left(101 \mathrm{MHz}, \mathrm{CDCl}_{3}\right) \delta 149.9,136.4,135.3,129.2,127.8,127.4,125.94,125.88$, 124.3, 112.5, 40.4, 21.2; HRMS (DART) $m / z$ calcd for $\mathrm{C}_{17} \mathrm{H}_{20} \mathrm{~N}[\mathrm{M}+\mathrm{H}]^{+}: 238.1590$ found 238.1587.

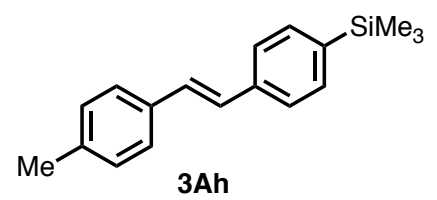

\section{(E)-Trimethyl(4-(4-methylstyryl)phenyl)silane (3Ah)}

Purification by PTLC (hexane/ $\mathrm{CH}_{2} \mathrm{Cl}_{2}=5: 1$ ) afforded $\mathbf{3 A h}$ as a white solid (20.0 $\mathrm{mg}, 38 \%$ yield). ${ }^{1} \mathrm{H}$ NMR (400 MHz, $\left.\mathrm{CDCl}_{3}\right) \delta 7.55-7.47(\mathrm{~m}, 4 \mathrm{H}), 7.42(\mathrm{~d}, J=7.2 \mathrm{~Hz}, 2 \mathrm{H}), 7.17(\mathrm{~d}, J=7.2 \mathrm{~Hz}, 2 \mathrm{H})$, $7.12(\mathrm{~d}, J=16.4 \mathrm{~Hz}, 1 \mathrm{H}), 7.06(\mathrm{~d}, J=16.4 \mathrm{~Hz}, 1 \mathrm{H}), 2.36(\mathrm{~s}, 3 \mathrm{H}), 0.28(\mathrm{~s}, 9 \mathrm{H}) ;{ }^{13} \mathrm{C} \mathrm{NMR}(101 \mathrm{MHz}$, $\left.\mathrm{CDCl}_{3}\right) \delta 139.7,137.9,137.5,134.5,133.7,129.4,128.8,127.6,126.4,125.7,21.3,-1.1$; HRMS (DART) $\mathrm{m} / z$ calcd for $\mathrm{C}_{18} \mathrm{H}_{23} \mathrm{Si}[\mathrm{M}+\mathrm{H}]^{+}: 267.1564$ found 267.1557 .

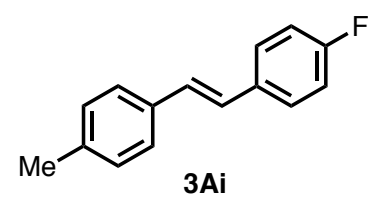

(E)-1-Fluoro-4-(4-methylstyryl)benzene (3Ai) ${ }^{[25]}$

Purification by PTLC (hexane/ $\mathrm{CHCl}_{3}=99: 1$ ) afforded $\mathbf{3 A i}$ as a white solid ( $26.8 \mathrm{mg}, 63 \%$ yield). ${ }^{1} \mathrm{H}$ NMR (400 MHz, $\left.\mathrm{CDCl}_{3}\right) \delta 7.50-7.43(\mathrm{~m}, 2 \mathrm{H}), 7.40(\mathrm{~d}, J=8.4 \mathrm{~Hz}, 2 \mathrm{H}), 7.17(\mathrm{~d}, J=8.4 \mathrm{~Hz}, 2 \mathrm{H})$, 7.08-6.95 (m, 4H), $2.36(\mathrm{~s}, 3 \mathrm{H}) ;{ }^{13} \mathrm{C} \mathrm{NMR}\left(101 \mathrm{MHz}, \mathrm{CDCl}_{3}\right) \delta 162.2\left(\mathrm{~d}, J_{\mathrm{C}-\mathrm{F}}=250.6 \mathrm{~Hz}\right), 137.6$, 134.3, 133.6, 129.4, 128.4, $127.8\left(\mathrm{~d}, J_{\mathrm{C}-\mathrm{F}}=7.8 \mathrm{~Hz}\right), 126.4\left(\mathrm{~d}, J_{\mathrm{C}-\mathrm{F}}=10.7 \mathrm{~Hz}\right), 126.3,115.5\left(\mathrm{~d}, J_{\mathrm{C}-\mathrm{F}}=\right.$ $22.3 \mathrm{~Hz}$ ), 21.2; HRMS (DART) $\mathrm{m} / z$ calcd for $\mathrm{C}_{15} \mathrm{H}_{13} \mathrm{~F}[\mathrm{M}]^{+}: 212.0996$ found 212.0993. 


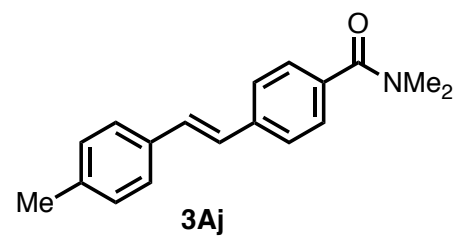

(E)-N,N-Dimethyl-4-(4-methylstyryl)benzamide (3Aj)

Purification by GPC afforded $\mathbf{3 A j}$ as a white solid $\left(27.5 \mathrm{mg}, 52 \%\right.$ yield). ${ }^{1} \mathrm{H}$ NMR (400 MHz, $\left.\mathrm{CDCl}_{3}\right) \delta 7.52(\mathrm{~d}, J=8.4 \mathrm{~Hz}, 2 \mathrm{H}), 7.46-7.36(\mathrm{~m}, 4 \mathrm{H}), 7.18(\mathrm{~d}, J=8.4 \mathrm{~Hz}, 2 \mathrm{H}), 7.13(\mathrm{~d}, J=16.4 \mathrm{~Hz}$, $1 \mathrm{H}), 7.05(\mathrm{~d}, J=16.4 \mathrm{~Hz}, 1 \mathrm{H}), 3.12(\mathrm{~s}, 3 \mathrm{H}), 3.02(\mathrm{~s}, 3 \mathrm{H}), 2.37(\mathrm{~s}, 3 \mathrm{H}) ;{ }^{13} \mathrm{C} \mathrm{NMR}\left(101 \mathrm{MHz}, \mathrm{CDCl}_{3}\right) \delta$ 171.4, 138.8, 137.9, 134.9, 134.1, 129.8, 129.4, 127.6, 126.7, 126.5, 126.1, 39.6, 35.4, 21.3; HRMS (ESI) $m / z$ calcd for $\mathrm{C}_{18} \mathrm{H}_{20} \mathrm{NO}[\mathrm{M}+\mathrm{H}]^{+}: 266.1539$ found 266.1536.

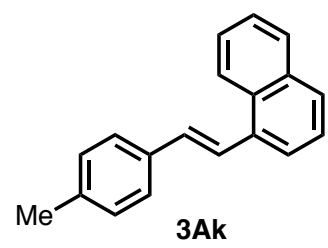

\section{(E)-1-(4-Methylstyryl)naphthalene (3Ak) ${ }^{[26]}$}

Purification by GPC afforded 3Ak as a white solid (30.0 mg, 61\% yield). ${ }^{1} \mathrm{H}$ NMR (400 MHz, $\left.\mathrm{CDCl}_{3}\right) \delta 8.22(\mathrm{~d}, J=8.0 \mathrm{~Hz}, 1 \mathrm{H}), 7.89-7.80(\mathrm{~m}, 2 \mathrm{H}), 7.78(\mathrm{~d}, J=8.0 \mathrm{~Hz}, 1 \mathrm{H}), 7.73(\mathrm{~d}, J=8.0 \mathrm{~Hz}$, 1H), 7.56-7.41 (m, 5H), $7.20(\mathrm{~d}, J=8.0 \mathrm{~Hz}, 2 \mathrm{H}), 7.12(\mathrm{~d}, J=16.0 \mathrm{~Hz}, 1 \mathrm{H}), 2.38(\mathrm{~s}, 3 \mathrm{H}) ;{ }^{13} \mathrm{C} \mathrm{NMR}$ $\left(101 \mathrm{MHz}, \mathrm{CDCl}_{3}\right) \delta 137.7,135.1,134.8,133.7,131.6,131.3,129.4,128.6,127.8,126.6,126.0,125.8$, 125.7, 124.7, 123.8, 123.4, 21.3; HRMS (DART) $\mathrm{m} / z$ calcd for $\mathrm{C}_{19} \mathrm{H}_{17}[\mathrm{M}+\mathrm{H}]^{+}: 245.1325$ found 245.1320 .

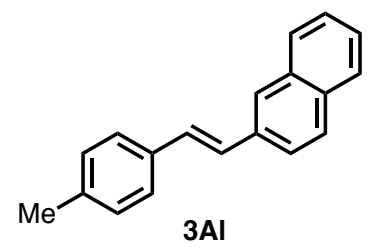

(E)-2-(4-Methylstyryl)naphthalene (3AI) ${ }^{[26]}$

Purification by PTLC (hexane/ $\mathrm{CHCl}_{3}=99: 1$ ) afforded $\mathbf{3 A l}$ as a white solid (34.2 $\mathrm{mg}, 70 \%$ yield). ${ }^{1} \mathrm{H}$ NMR (400 MHz, $\mathrm{CDCl}_{3}$ ) $\delta$ 7.87-7.76 (m, 4H), 7.73 (dd, $\left.J=8.4,1.2 \mathrm{~Hz}, 1 \mathrm{H}\right), 7.49-7.40(\mathrm{~m}, 4 \mathrm{H})$, 7.23-7.16 (m, 4H), 2.37 (s, 3H); ${ }^{13} \mathrm{C}$ NMR (101 MHz, $\left.\mathrm{CDCl}_{3}\right) \delta 137.6,135.0,134.5,133.7,132.9$, $129.4,128.9,128.2,127.9,127.74,127.66,126.43,126.38,126.3,125.8,123.5,21.3$; HRMS (DART) $m / z$ calcd for $\mathrm{C}_{19} \mathrm{H}_{17}[\mathrm{M}+\mathrm{H}]^{+}: 245.1325$ found 245.1320 . 


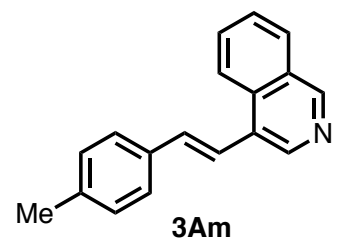

\section{(E)-4-(4-Methylstyryl)isoquinoline (3Am)}

Purification by GPC afforded 3Am as a white solid (19.2 mg, 39\% yield). ${ }^{1} \mathrm{H}$ NMR (400 MHz, $\left.\mathrm{CDCl}_{3}\right) \delta 9.18(\mathrm{~s}, 1 \mathrm{H}), 8.76(\mathrm{~s}, 1 \mathrm{H}), 8.16(\mathrm{~d}, J=8.0 \mathrm{~Hz}, 1 \mathrm{H}), 7.99(\mathrm{~d}, J=7.6 \mathrm{~Hz}, 1 \mathrm{H}), 7.74(\mathrm{dd}, J=$ 8.0, 7.6 Hz, 1H), 7.69-7.59 (m, 2H), 7.51 (d, $J=8.4 \mathrm{~Hz}, 2 \mathrm{H}), 7.22(\mathrm{~d}, J=8.4 \mathrm{~Hz}, 2 \mathrm{H}), 7.18(\mathrm{~d}, J=$ $16.4 \mathrm{~Hz}, 1 \mathrm{H}), 2.39$ (s, 3H); ${ }^{13} \mathrm{C}$ NMR (101 MHz, $\left.\mathrm{CDCl}_{3}\right) \delta 151.6,140.3,138.2,134.3,133.7,133.1$, $130.4,129.5,128.8,128.1,127.1,126.7,122.9,121.3,21.3$ (one peak is missing due to overlapping); HRMS (ESI) $m / z$ calcd for $\mathrm{C}_{18} \mathrm{H}_{16} \mathrm{~N}[\mathrm{M}+\mathrm{H}]^{+}: 246.1277$ found 246.1275.
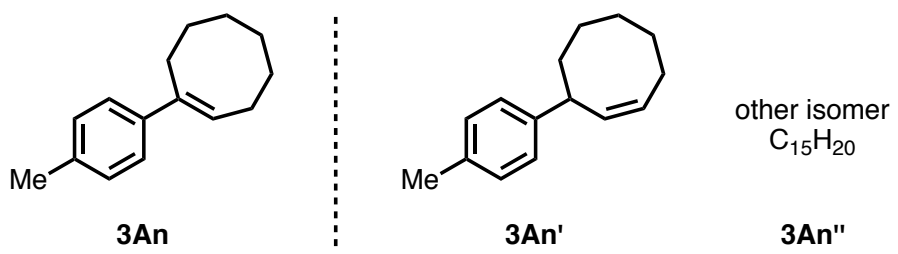

\section{$(E)-1-(p-T o l y l) c y c l o o c t-1-e n e ~(3 A n)^{[27]}$}

Purification by PTLC (hexane/ $\mathrm{CH}_{2} \mathrm{Cl}_{2}=9: 1$ ) afforded a mixture of $\mathbf{3 A n},{ }^{[27]}$ (Z)-3-( $p$-tolyl)cyclooct-1-ene (3An') and other isomer (3An"') as a colorless oil (20.9 $\mathrm{mg}, 52 \%$ yield). A ratio of 3An, 3An', and 3An" was determined as 81:12:7 ( ${ }^{1} \mathrm{H}$ NMR peaks at $5.98 \mathrm{ppm}(\mathrm{t}, J=8.4 \mathrm{~Hz}$, 1H, 3An), 5.81-5.63 ppm (m, 2H, 3An'), and 5.62-5.53 ppm (m, 2H, 3An") were used, respectively) and the yield of $\mathbf{3 A n}$ was determined as $42 \%$. Further purification by GPC was performed to give pure 3An as a colorless oil for the characterization. ${ }^{1} \mathrm{H}$ NMR $\left(400 \mathrm{MHz}, \mathrm{CDCl}_{3}\right) \delta 7.31(\mathrm{~d}, J=8.4 \mathrm{~Hz}, 2 \mathrm{H})$, $7.11(\mathrm{~d}, J=8.4 \mathrm{~Hz}, 2 \mathrm{H}), 5.98(\mathrm{t}, J=8.4 \mathrm{~Hz}, 1 \mathrm{H}), 2.69-2.58(\mathrm{~m}, 2 \mathrm{H}), 2.33(\mathrm{~s}, 3 \mathrm{H}), 2.32-2.24(\mathrm{~m}, 2 \mathrm{H})$, $1.78-1.48(\mathrm{~m}, 8 \mathrm{H}) ;{ }^{13} \mathrm{C} \mathrm{NMR}\left(101 \mathrm{MHz}, \mathrm{CDCl}_{3}\right) \delta 140.2,139.9,136.0,128.9,127.1,125.6,30.0,29.4$, 28.3, 27.4, 26.9, 26.1, 21.0; HRMS (DART) $m / z$ calcd for $\mathrm{C}_{15} \mathrm{H}_{21}[\mathrm{M}+\mathrm{H}]^{+}: 201.1638$ found 201.1636.

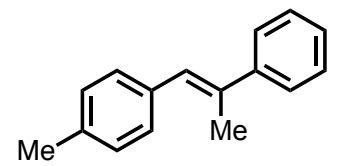

(E)-3Ao

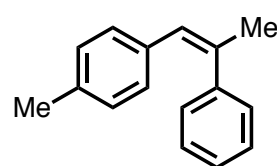

(Z)-3Ao

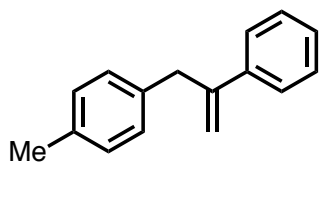

3Ao'

1-Methyl-4-(2-phenylprop-1-en-1-yl)benzene (3Ao) and 1-methyl-4-(2-phenylallyl)benzene $\left(\mathbf{B A o}^{\prime}\right)^{[28,29]}$

Purification by PTLC (hexane/ $\mathrm{CH}_{2} \mathrm{Cl}_{2}=9: 1$ ) afforded an inseparable mixture of $(E)$-3Ao, $(Z)$-3Ao and 3Ao' as a colorless oil (36.4 mg, $0.80 \mathrm{mmol} \mathrm{scale,} 22 \%$ yield). A ratio of (E)-3Ao, $(Z)-\mathbf{3 A o}$ and 3Ao' was determined as 42:25:33 ( ${ }^{1} \mathrm{H}$ NMR peaks at $7.52 \mathrm{ppm}(\mathrm{d}, J=7.6 \mathrm{~Hz}, 2 \mathrm{H},(E)-3 \mathrm{Ao}), 6.43 \mathrm{ppm}$ 
(s, 1H, (Z)-3Aoo) and $5.48 \mathrm{ppm}\left(\mathrm{d}, J=0.8 \mathrm{~Hz}, 1 \mathrm{H}, \mathbf{3} \mathbf{A o}^{\prime}\right)$ were used, respectively) and the yield of (E)-3Ao was determined as $9 \%$.

For (E)-3Ao ${ }^{[28]}:{ }^{1} \mathrm{H}$ NMR (400 MHz, $\left.\mathrm{CDCl}_{3}\right) \delta 7.52(\mathrm{~d}, J=7.6 \mathrm{~Hz}, 2 \mathrm{H}), 7.36(\mathrm{t}, J=7.6 \mathrm{~Hz}, 2 \mathrm{H}), 7.31-$ $7.15(\mathrm{~m}, 5 \mathrm{H}), 6.81(\mathrm{~s}, 1 \mathrm{H}), 2.37(\mathrm{~s}, 3 \mathrm{H}), 2.28(\mathrm{~s}, 3 \mathrm{H}) ;{ }^{13} \mathrm{C} \mathrm{NMR}\left(101 \mathrm{MHz}, \mathrm{CDCl}_{3}\right) \delta 144.1,136.7$, 136.1, 135.4, 129.04, 128.9, 128.3, 127.6, 127.0, 125.9, 21.2, 17.5.

For (Z)-3Ao ${ }^{[28]}:{ }^{1} \mathrm{H}$ NMR (400 MHz, $\left.\mathrm{CDCl}_{3}\right) \delta 7.31-7.15(\mathrm{~m}, 5 \mathrm{H}), 6.90(\mathrm{~d}, J=8.4 \mathrm{~Hz}, 2 \mathrm{H}), 6.83(\mathrm{~d}, J$ $=8.4 \mathrm{~Hz}, 2 \mathrm{H}), 6.43(\mathrm{~s}, 1 \mathrm{H}), 2.23(\mathrm{~s}, 3 \mathrm{H}), 2,19(\mathrm{~s}, 3 \mathrm{H}),{ }^{13} \mathrm{C} \mathrm{NMR}\left(101 \mathrm{MHz}, \mathrm{CDCl}_{3}\right) \delta 142.3,137.8$, $135.7 ; 134.6 ; 128.6,128.4 ; 128.1,126.8,126.4,27.1,21.1$ (one peak is missing due to overlapping).

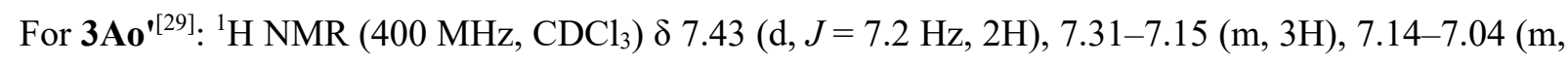
$4 \mathrm{H}), 5.48(\mathrm{~d}, J=0.8 \mathrm{~Hz}, 1 \mathrm{H}), 5.01(\mathrm{~d}, J=0.8 \mathrm{~Hz}, 1 \mathrm{H}), 3.79(\mathrm{~s}, 2 \mathrm{H}), 2.30(\mathrm{~s}, 3 \mathrm{H}) ;{ }^{13} \mathrm{C} \mathrm{NMR}(101 \mathrm{MHz}$, $\left.\mathrm{CDCl}_{3}\right) \delta 147.1,140.8,136.4,135.5,129.00,128.8,128.2,127.4,126.1,114.4,41.1,21.0$.

HRMS (DART) of the mixture; $m / z$ calcd for $\mathrm{C}_{16} \mathrm{H}_{17}[\mathrm{M}+\mathrm{H}]^{+}: 209.1325$ found 209.1323. 


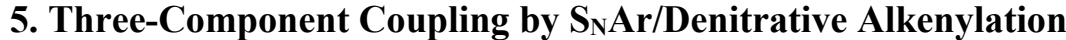
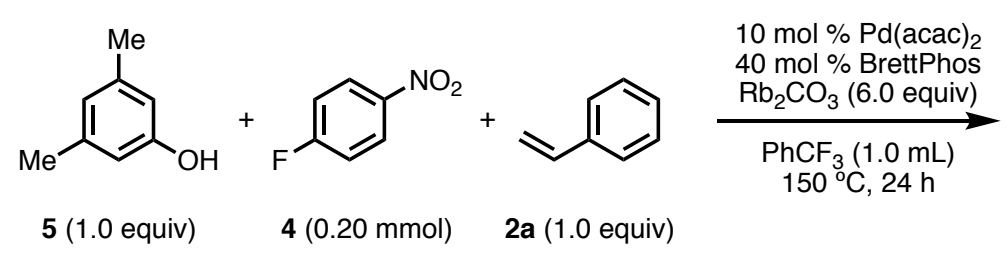

5 (1.0 equiv) $\quad \mathbf{4}(0.20 \mathrm{mmol}) \quad \mathbf{2 a}(1.0$ equiv $)$

A $20-\mathrm{mL}$ glass vessel equipped with J. Young ${ }^{\circledR}$ O-ring tap containing a magnetic stirring bar and $\mathrm{Rb}_{2} \mathrm{CO}_{3}$ (277 mg, $1.2 \mathrm{mmol}$, 6.0 equiv) was dried with a heat-gun in vacuo and filled with $\mathrm{N}_{2}$ after cooling to room temperature. To this vessel were added 3,5-dimethylphenol (5: $24.4 \mathrm{mg}, 0.20 \mathrm{mmol}$, 1.0 equiv), 2-dicyclohexylphosphino-3,6-dimethoxy-2',4',6'-triisopropylbiphenyl (BrettPhos: 42.9 mg, $0.080 \mathrm{mmol}, 40 \mathrm{~mol} \%)$ and $\mathrm{Pd}(\mathrm{acac})_{2}(6.1 \mathrm{mg}, 0.020 \mathrm{mmol}, 10 \mathrm{~mol} \%)$. The vessel was placed under vacuum and refilled $\mathrm{N}_{2}$ gas three times, and then added 1-fluoro-4-nitrobenzene (4: $28.2 \mathrm{mg}, 21.2 \mu \mathrm{L}$, $0.20 \mathrm{mmol}$ ), styrene (2a: $20.8 \mathrm{mg}, 22.9 \mu \mathrm{L}, 0.20 \mathrm{mmol}, 1.0$ equiv), and (trifluoromethyl)benzene $\left(\mathrm{PhCF}_{3}: 1.0 \mathrm{~mL}\right)$. The vessel was sealed with O-ring tap and then heated at $150{ }^{\circ} \mathrm{C}$ for $24 \mathrm{~h}$ with stirring. After cooling the reaction mixture to room temperature, the mixture was passed through a short silica-gel/Celite ${ }^{\circledR}(1: 1)$ pad with EtOAc as an eluent, and then concentrated in vacuo. The residue was purified by PTLC (hexane/ $\mathrm{CH}_{2} \mathrm{Cl}_{2}=19: 1$ ) to afford (E)-1,3-dimethyl-5-(4-styrylphenoxy)benzene (6: $24.6 \mathrm{mg}, 41 \%$ yield) as a white solid. ${ }^{1} \mathrm{H} \mathrm{NMR}\left(\mathrm{CDCl}_{3}, 400 \mathrm{MHz}\right) \delta 7.54-7.46(\mathrm{~m}, 4 \mathrm{H}), 7.38-7.34$ (m, 3H), 7.10 (d, $J=16.0 \mathrm{~Hz}, 1 \mathrm{H}), 7.02$ (d, $J=16.0 \mathrm{~Hz}, 1 \mathrm{H}), 6.99$ (d, $J=8.8 \mathrm{~Hz}, 2 \mathrm{H}), 6.77$ (s, 1H), $6.66(\mathrm{~s}, 2 \mathrm{H}), 2.27(\mathrm{~s}, 6 \mathrm{H}) ;{ }^{13} \mathrm{C} \mathrm{NMR}\left(\mathrm{CDCl}_{3}, 101 \mathrm{MHz}\right) \delta 157.1,157.0,139.6,137.4,132.3,128.7$, $127.9,127.8,127.6,127.4,126.3,125.1,118.9,116.6,21.3$; HRMS (DART) $\mathrm{m} / z$ calcd for $\mathrm{C}_{22} \mathrm{H}_{21} \mathrm{O}$ $[\mathrm{M}+\mathrm{H}]^{+}: 301.1587$ found 301.1583 . 


\section{Effect of Parameters}

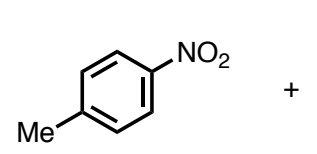

$1 \mathrm{~A}$ (1.5 equiv)

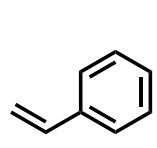

$2 a(0.20 \mathrm{mmol})$

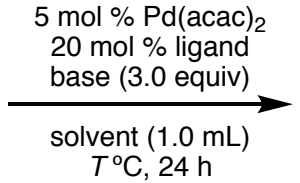

$T^{\circ} \mathrm{C}, 24 \mathrm{~h}$<smiles>Cc1ccc(/C=C/c2ccccc2)cc1</smiles>

3Aa

GC Yield of 3Aa (determined based on the amount of 2a)

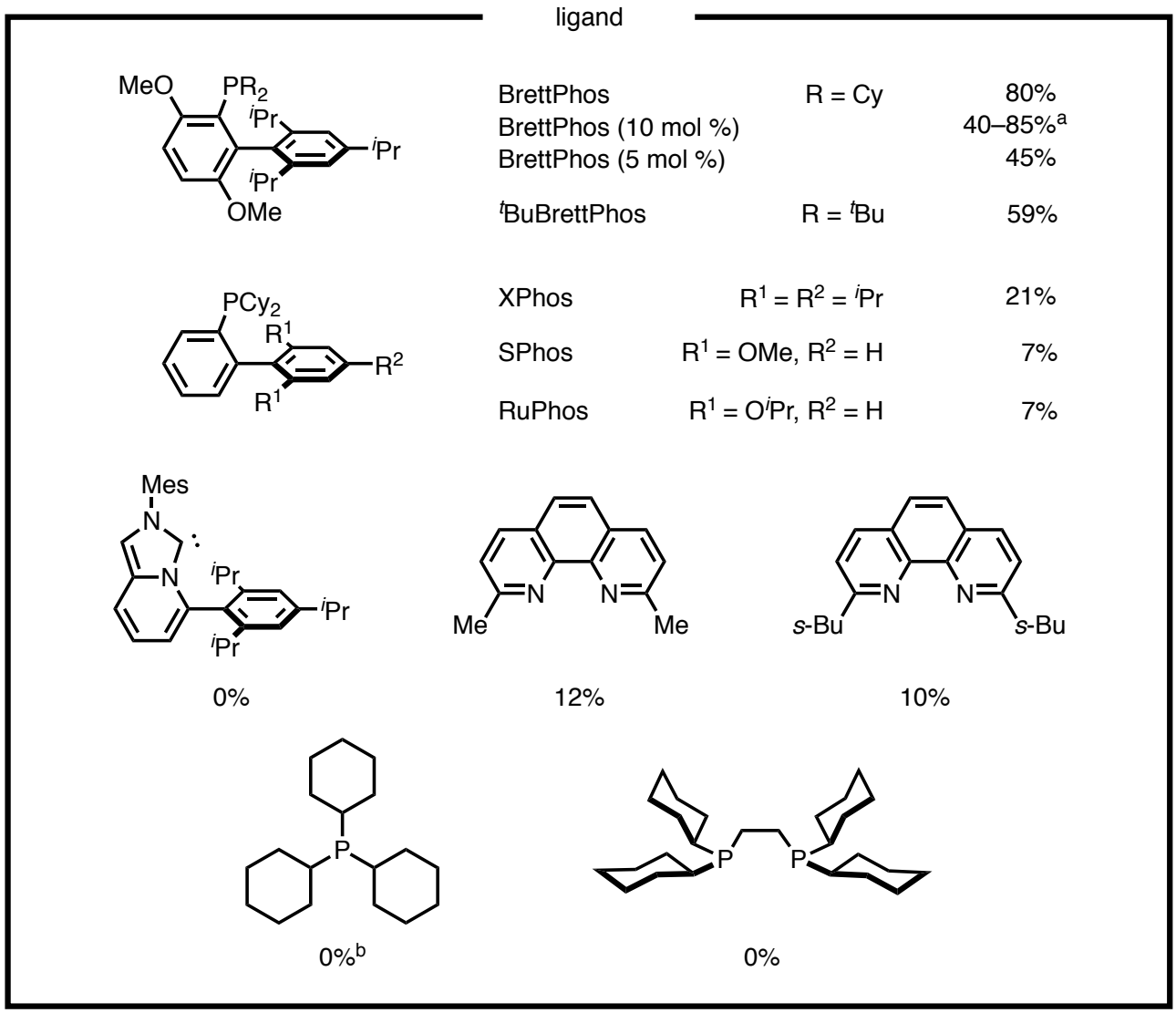

base: $\mathrm{Rb}_{2} \mathrm{CO}_{3}$, solvent: $\mathrm{PhCF}_{3}, T=150^{\circ} \mathrm{C}$

a Low reproducibility ${ }^{\mathrm{b}} 40 \mathrm{~mol} \%$ as $0.60 \mathrm{M}$ solution in toluene.

\begin{tabular}{|cc|}
\hline solvent & \\
\cline { 2 - 2 } nhexane & $4 \%$ \\
cyclohexane & $12 \%$ \\
1,4-dioxane & $8 \%$ \\
benzene & $41 \%$ \\
toluene & $20 \%$ \\
m-xylene & $52 \%$ \\
anisole & $40 \%$ \\
PhCF & $53 \%$ \\
o-dichlorobenzene & $26 \%$ \\
mesitylene & $45 \%$ \\
$\mathrm{THF}$ & $4 \%$ \\
$\mathrm{DMF}^{\mathrm{BMH}}$ & $7 \%$ \\
$\mathrm{BH}_{2} \mathrm{Cl}_{2}$ & $18 \%$ \\
$\mathrm{CHCl}_{3}$ & $0 \%$ \\
1,4-dioxane & $0 \%$ \\
toluene $^{\mathrm{c}}$ & $30 \%$ \\
$\mathrm{THF}^{\mathrm{c}}$ & $56 \%$ \\
& $11 \%$ \\
\hline
\end{tabular}

ligand: BrettPhos, base: $\mathrm{CsF}, T=150^{\circ} \mathrm{C}$ ${ }^{c} \mathrm{Rb}_{2} \mathrm{CO}_{3}$ was used as a base.

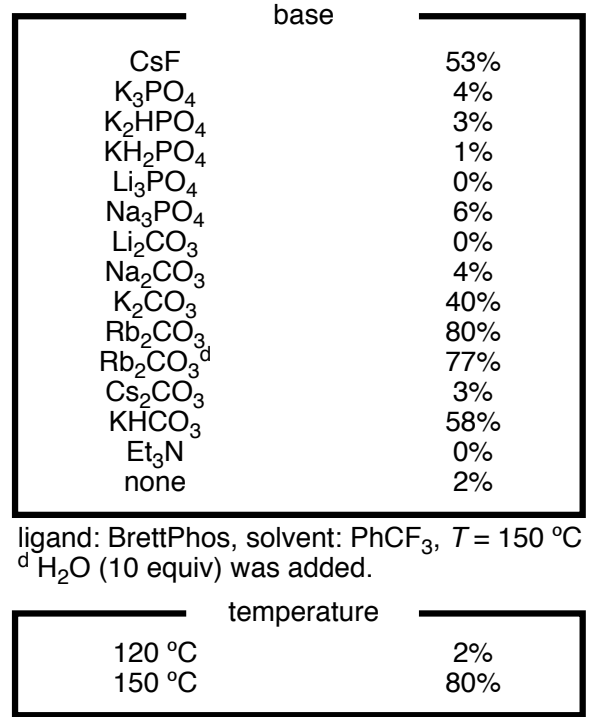

ligand: BrettPhos, base: $\mathrm{Rb}_{2} \mathrm{CO}_{3}$, solvent: $\mathrm{PhCF}_{3}$ 


\section{References}

[1] Yang, T.; Liu, Q.; Cheng, Y.; Cai, W.; Ma, Y.; Yang, L.; Wu, Q.; Orband-Miller, L. A.; Zhou, L.; Xiang, Z.; Huxdorf, M.; Zhang, W.; Zhang, J.; Xiang, J.-N.; Leung, S.; Qiu, Y.; Zhong, Z.; Elliott, J. D.; Lin, X.; Wang, Y. Discovery of Tertiary Amine and Indole Derivatives as Potent ROR $\gamma$ Inverse Agonists. ACS Med. Chem. Lett. 2014, 5, 65-68.

[2] Lane, B. S.; Sames, D. Direct C-H Bond Arylation: Selective Palladium-Catalyzed C2-Arylation of $\mathrm{N}$-Substituted Indoles. Org. Lett. 2004, 6, 2897-2900.

[3] Song, C.; Chen, P.; Tang, Y. Carboxylation of Styrenes with $\mathrm{CBr}_{4}$ and DMSO via Cooperative Photoredox and Cobalt Catalysis. RSC Adv. 2017, 7, 11233-11243.

[4] Terent'ev, A. O.; Mulina, O. M.; Pirgach, D. A.; Ilovaisky, A. I.; Syroeshkin, M. A.; Kapustina, N. I.; Nikishin, G. I. Electrosynthesis of Vinyl Sulfones from Alkenes and Sulfonyl Hydrazides Mediated by KI: An Electrochemical Mechanistic Study. Tetrahedron 2017, 73, 6871-6879.

[5] Peng, J.; Docherty, J. H.; Dominey, A. P.; Thomas, S. P. Cobalt-Catalysed Markovnikov Selective Hydroboration of Vinylarenes. Chem. Commun. 2017, 53, 4726-4729.

[6] Lee J.; Seo, M. Hyper-Cross-Linked Polymer with Enhanced Porosity by in situ Removal of Trimethylsilyl Group via Electrophilic Aromatic Substitution. ACS Macro Lett. 2018, 7, 1448-1454.

[7] Landge, V. G.; Yadav, V.; Subaramanian, M.; Dangarh, P.; Balaraman, E. Nickel(II)-Catalyzed Direct Olefination of Benzyl Alcohols with Sulfones with the Liberation of $\mathrm{H}_{2}$. Chem. Commun. 2019, $55,6130-6133$.

[8] Louaisil, N.; Pham, P. D.; Boeda, F.; Faye, D.; Castanet, A.-S.; Legoupy, S. Ionic Liquid Supported Organotin Reagents: Green Tools for Stille Cross-Coupling Reactions with Brominated Substrates. Eur. J. Org. Chem. 2011, 143-149.

[9] Mäsing, F.; Mardyukov, A.; Doerenkamp, C.; Eckert, H.; Malkus, U.; Nüsse, H.; Klingauf, J.; Studer, A. Controlled Light-Mediated Preparation of Gold Nanoparticles by a Norrish Type I Reaction of Photoactive Polymers. Angew. Chem., Int. Ed. 2015, 54, 12612-12617.

[10] Yao, X.; Shao, Y.; Hu, M.; Xia, Y.; Cheng, T.; Chen, J. Palladium-Catalyzed Cascade Reaction of $o$-Cyanobiaryls with Arylboronic Acids: Synthesis of 5-Arylidene-7-aryl-5H-dibenzo[c,e]-azepines. Org. Lett. 2019, 21, 7697-7701.

[11] Wang, S.-M.; Song, H.-X.; Wang, X.-Y.; Liu, N.; Qin, H.-L.; Zhang, C.-P. Palladium-Catalyzed Mizoroki-Heck-Type Reactions of $\left[\mathrm{Ph}_{2} \mathrm{SR}_{\mathrm{fn}}\right][\mathrm{OTf}]$ with Alkenes at Room Temperature. Chem. Commun. 2016, 52, 11893-11896.

[12] Schroeter, F.; Soellner, J.; Strassner, T. Cross-Coupling Catalysis by an Anionic Palladium Complex. ACS Catal. 2017, 7, 3004-3009.

[13] Prasanna, R.; Guha, S.; Sekar, G. Proton-Coupled Electron Transfer: Transition-Metal-Free Reduction of Chalcones and Alkynes Using Xanthate/Formic Acid. Org. Lett. 2019, 21, 2650-2653. 
[14] Takahashi, K.; Morishita, H.; Ogiwara, Y.; Sakai, N. Group 4 Metallocene Difluoride/Palladium Bimetallic Catalysts for the Reductive Cross-Coupling of Alkynes with Aryl Iodides and Bromides. $J$. Org. Chem. 2018, 83, 13734-13742.

[15] Satoh, T.; Jones, W. D. Palladium-Catalyzed Coupling Reactions of Biphenylene with Olefins, Arylboronic Acids, and Ketones Involving C-C Bond Cleavage. Organometallics 2001, 20, 29162919.

[16] Hostier, T.; Neouchy, Z.; Ferey, V.; Gomez Pardo, D.; Cossy, J. Nickel-Catalyzed System for the Cross-Coupling of Alkenyl Methyl Ethers with Grignard Reagents under Mild Conditions. Org. Lett. 2018, 20, 1815-1818.

[17] Adam, R.; Cabrero-Antonino, J. R.; Spannenberg, A.; Junge, K.; Jackstell, R.; Beller, M. A General and Highly Selective Cobalt-Catalyzed Hydrogenation of N-Heteroarenes under Mild Reaction Conditions. Angew. Chem., Int. Ed. 2017, 56, 3216-3220.

[18] Ha, P. T.; Nguyen, O. T. K.; Huynh, K. D.; Nguyen T. T.; Phan, N. T. S. Synthesis of Unnatural Arundines Using a Magnetically Reusable Copper Ferrite Catalyst. Synlett 2018, 29, 2031-2034.

[19] Nojima, M.; Ohta, Y.; Yokozawa, T. Structural Requirements for Palladium Catalyst Transfer on a Carbon-Carbon Double Bond. J. Am. Chem. Soc. 2015, 137, 5682-5685.

[20] Kadari, L.; Palakodety, R. K. Pd/Cu-Catalyzed Facile Approach to Stilbenes: A Novel Diversity of TosMIC as an Aryl Source. Tetrahedron Lett. 2019, 60, 1978-1981.

[21] Lee, J.-Y.; Su, Y.-S.; Wang, Y.-S.; Lee, H. M. Tetranuclear Palladium Complexes of Abnormal $\mathrm{N}$-Heterocyclic Carbene Ligands and their Catalytic Activities in Mizoroki-Heck Coupling Reaction of Electron-Rich Aryl Chlorides. Adv. Synth. Catal. 2019, 361, 4714-4726.

[22] Lebel, H.; Ladjel, C.; Bréthous, L. Palladium-Catalyzed Cross-Coupling Reactions in One-Pot Multicatalytic Processes. J. Am. Chem. Soc. 2007, 129, 13321-13326.

[23] Keesara, S.; Parvathaneni, S.; Dussa, G.; Mandapati, M. R. Polystyrene Supported Thiopseudourea Pd(II) Complex: Applications for Sonogashira, Suzuki-Miyaura, Heck, Hiyama and Larock Heteroannulation Reactions. J. Organomet. Chem. 2014, 765, 31-38.

[24] Katritzky, A. R.; Cheng, D.; Li, J. Benzotriazole-Mediated Stereoselective Olefination of Carboxylic Esters: Transformation of $\alpha$-Amino Acid Esters into Chiral Allylamines. J. Org. Chem. 1998, 63, 3438-3444.

[25] Taniguchi, N. Aerobic Palladium-Catalyzed Arylation of Alkenes Using Sodium Sulfinates. Synlett 2013, 24, 2571-2574.

[26] Keesara, S.; Parvathaneni, S.; Mandapati, M. R. N,N'-Mono Substituted Acyclic Thioureas: Efficient Ligands for the Palladium Catalyzed Heck Reaction of Deactivated Aryl Bromides. Tetrahedron Lett. 2014, 55, 6769-6772.

[27] Kamigata, N.; Satoh, A.; Kondoh, T.; Kameyama, M. Arylation of Olefins by Arylazo Aryl Sulfones under Palladium(0) Catalysis. Bull. Chem. Soc. Jpn. 1988, 61, 3575-3580. 
[28] Ho, G.-M.; Sommer H.; Marek I. Highly E-Selective, Stereoconvergent Nickel-Catalyzed Suzuki-Miyaura Cross-Coupling of Alkenyl Ethers. Org. Lett. 2019, 21, 2913-2917.

[29] Hou, Z.-L.; Yang, F.; Zhou, Z.; Ao, Y.-F.; Yao, B. Silver-Promoted Cross-Coupling of Substituted Allyl(trimethyl)silanes with Aryl Iodides by Palladium Catalysis. Tetrahedron Lett. 2018, $59,4557-4561$. 


\section{8. ${ }^{1} \mathrm{H}$ and ${ }^{13} \mathrm{C}$ NMR Spectra}

${ }^{1} \mathrm{H}$ NMR of $\mathbf{1 N}\left(400 \mathrm{MHz}, \mathrm{CDCl}_{3}\right)$

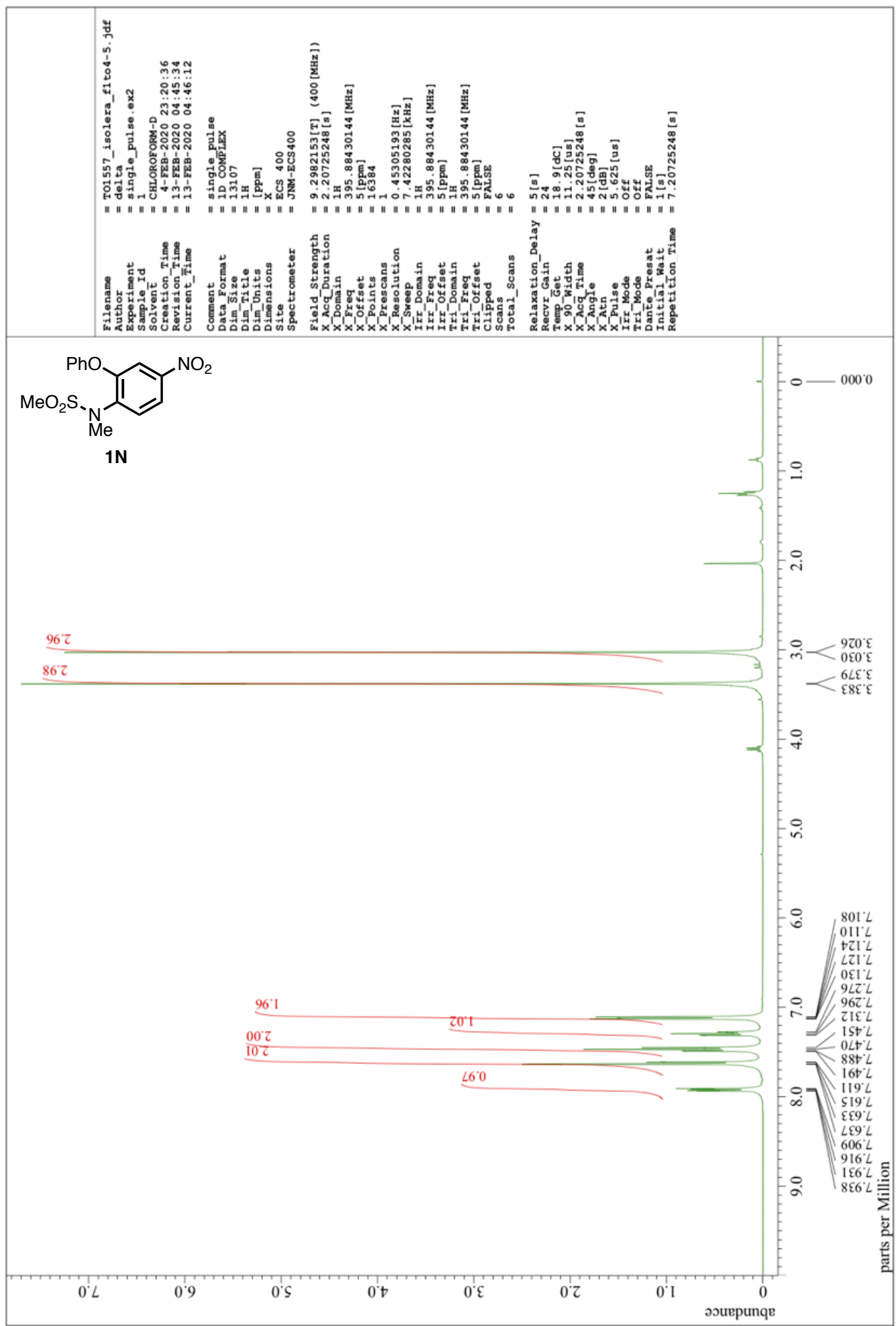


${ }^{13} \mathrm{C}$ NMR of $\mathbf{1 N}\left(101 \mathrm{MHz}, \mathrm{CDCl}_{3}\right)$

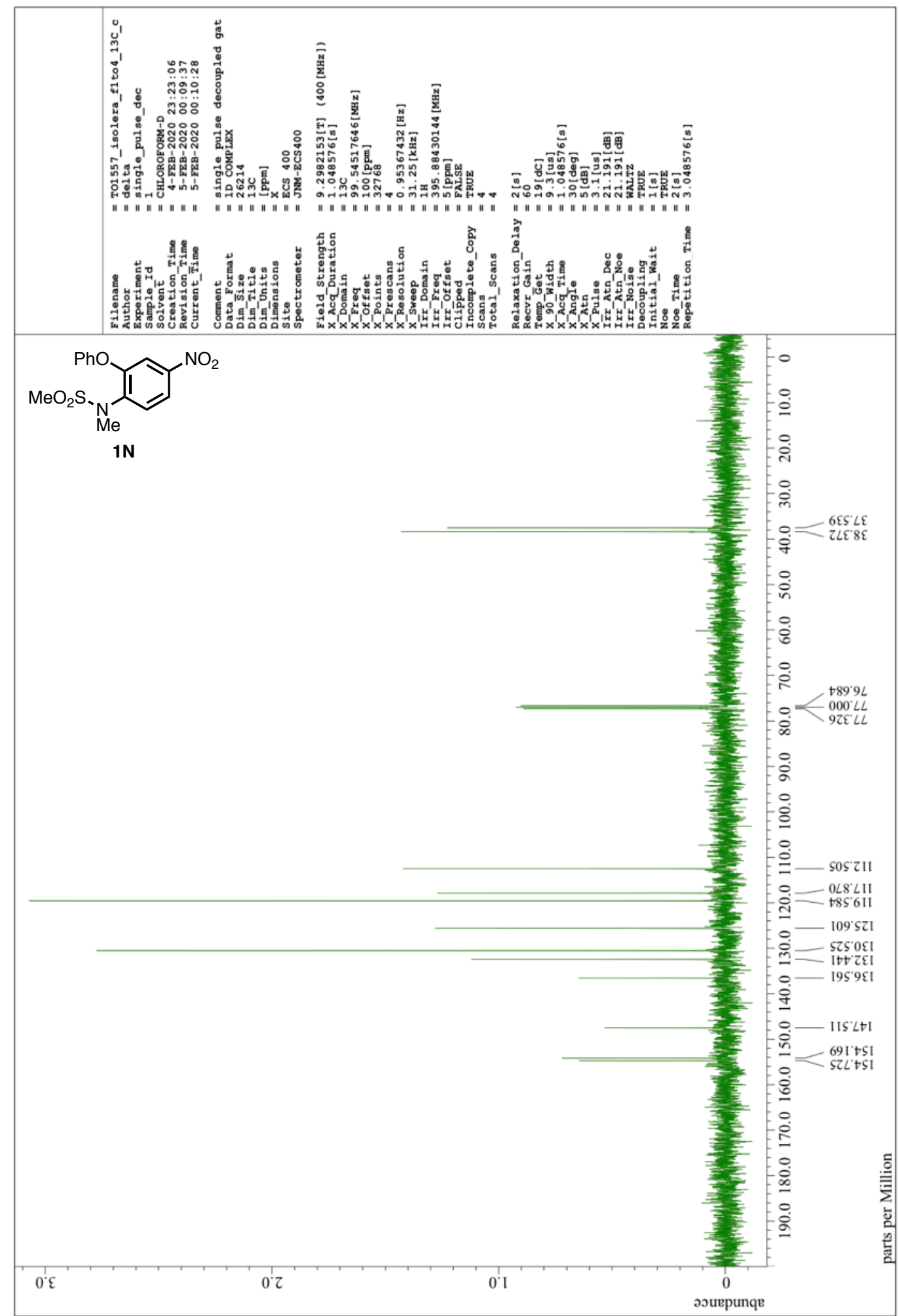


${ }^{1} \mathrm{H}$ NMR of $\mathbf{3 A a}\left(400 \mathrm{MHz}, \mathrm{CDCl}_{3}\right)$

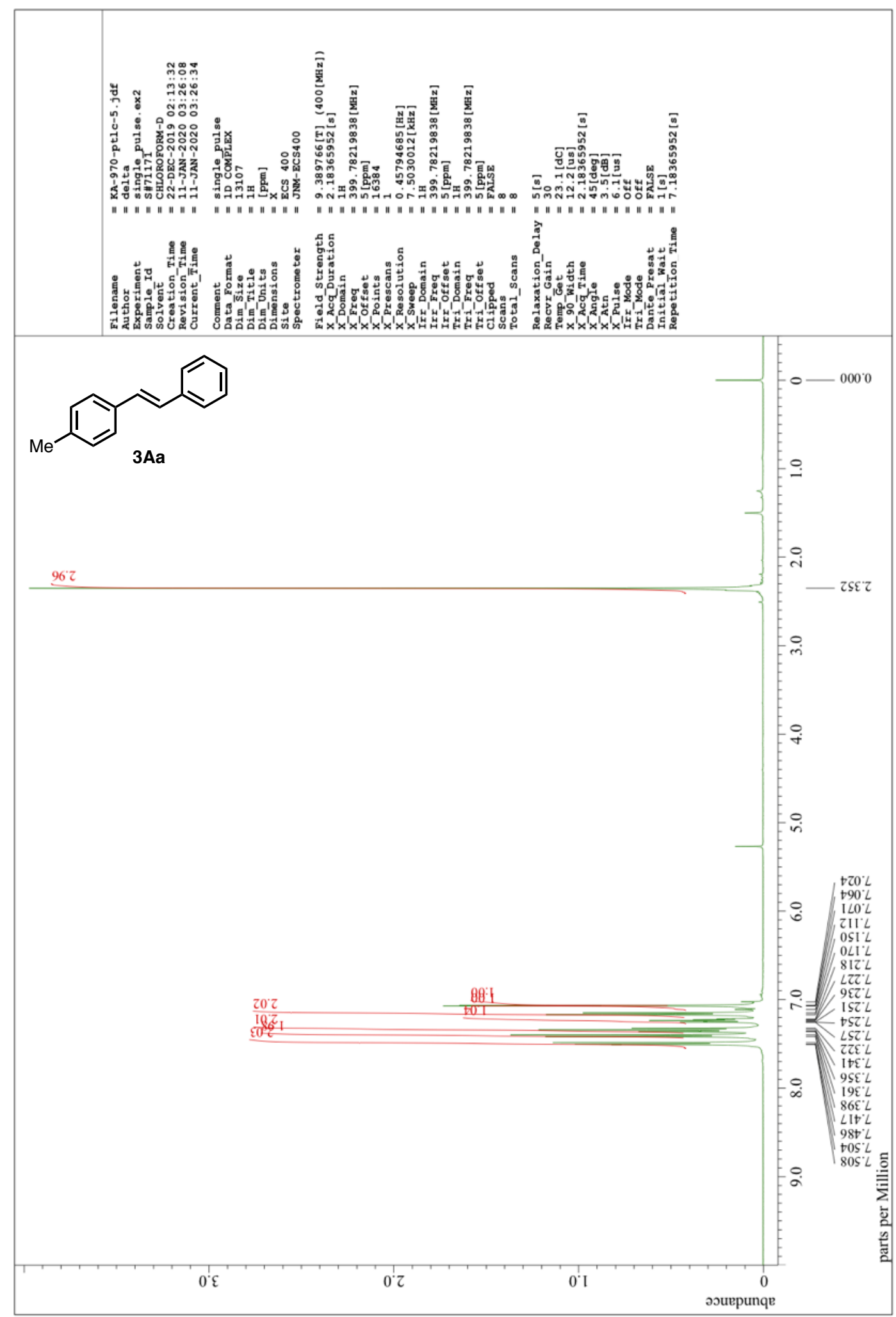


${ }^{13} \mathrm{C}$ NMR of $3 \mathrm{Aa}\left(101 \mathrm{MHz}, \mathrm{CDCl}_{3}\right)$

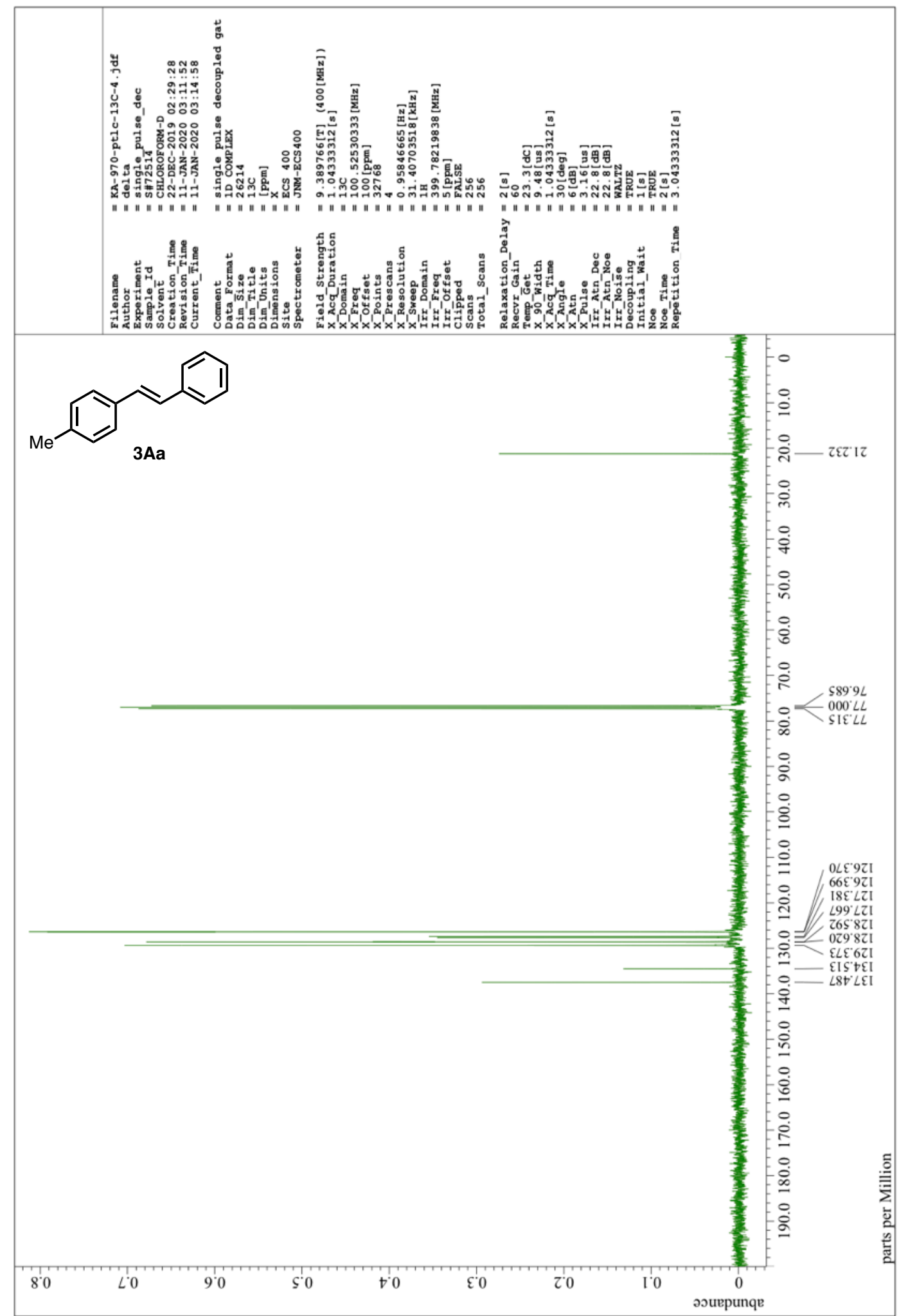


${ }^{1} \mathrm{H}$ NMR of 3Ba $\left(400 \mathrm{MHz}, \mathrm{CDCl}_{3}\right)$

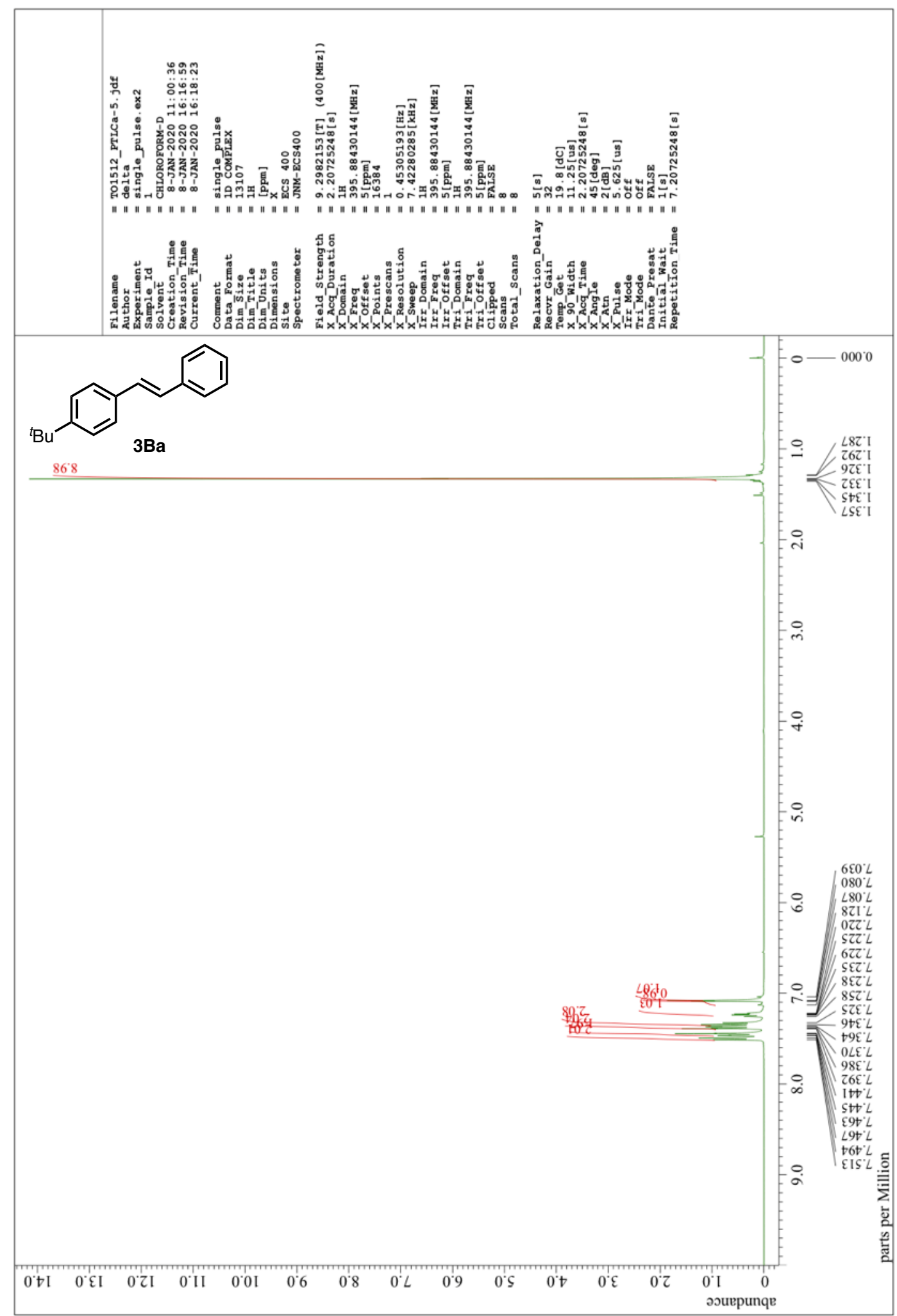


${ }^{13} \mathrm{C}$ NMR of $\mathbf{3 B a}\left(101 \mathrm{MHz}, \mathrm{CDCl}_{3}\right)$

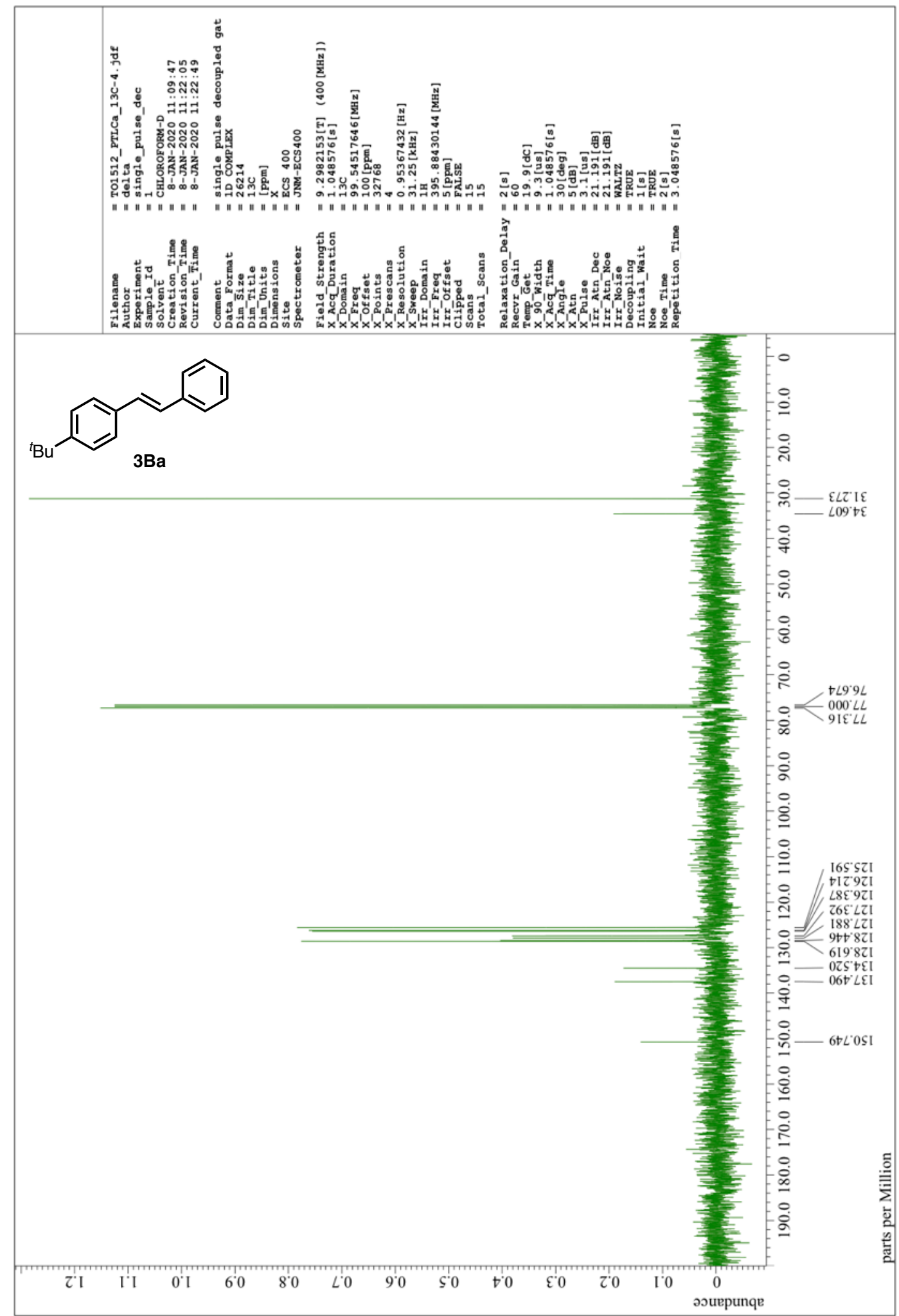


${ }^{1} \mathrm{H}$ NMR of $\mathbf{3 C a}\left(400 \mathrm{MHz}, \mathrm{CDCl}_{3}\right)$

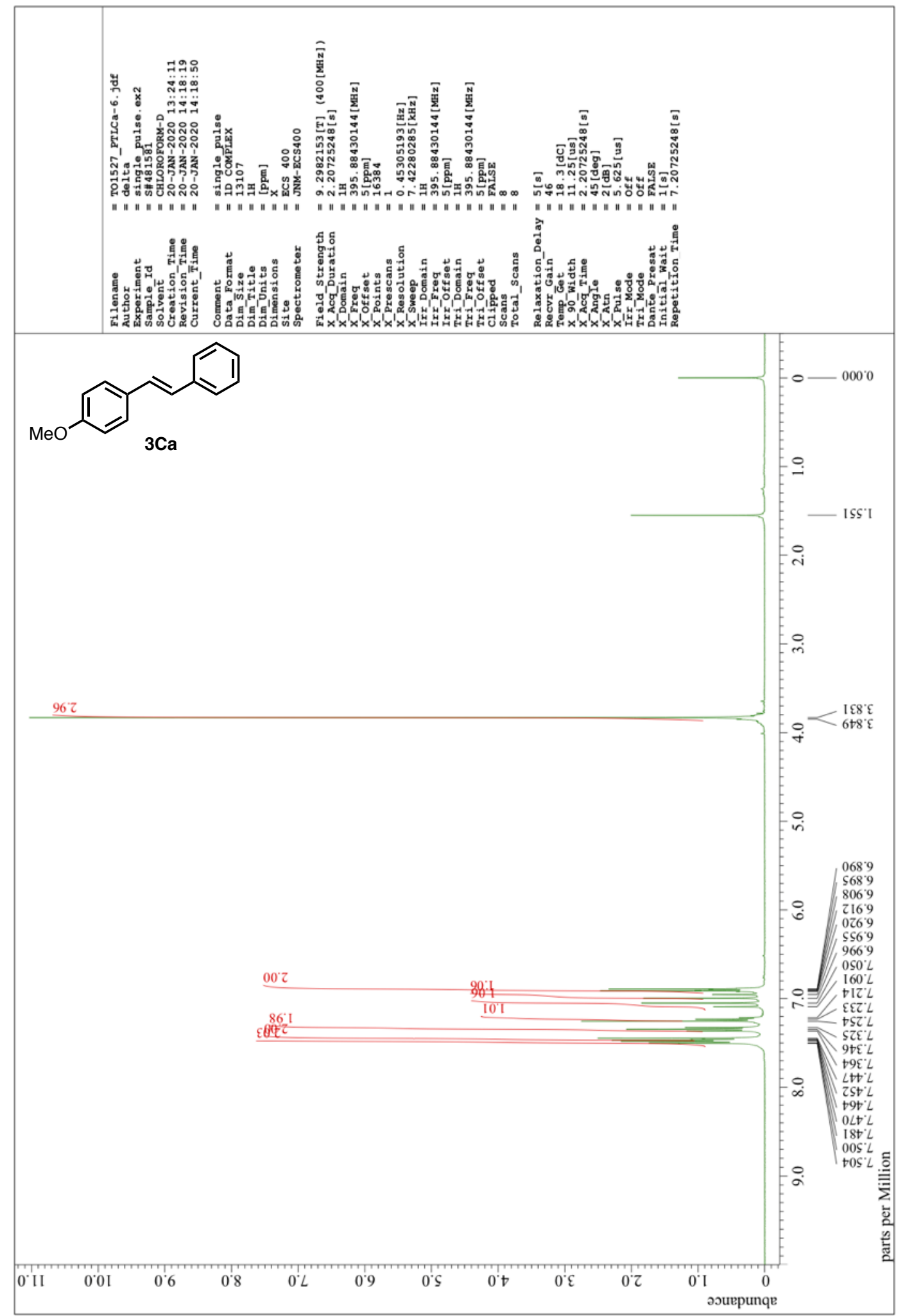


${ }^{13} \mathrm{C}$ NMR of $\mathbf{3 C a}\left(101 \mathrm{MHz}, \mathrm{CDCl}_{3}\right)$

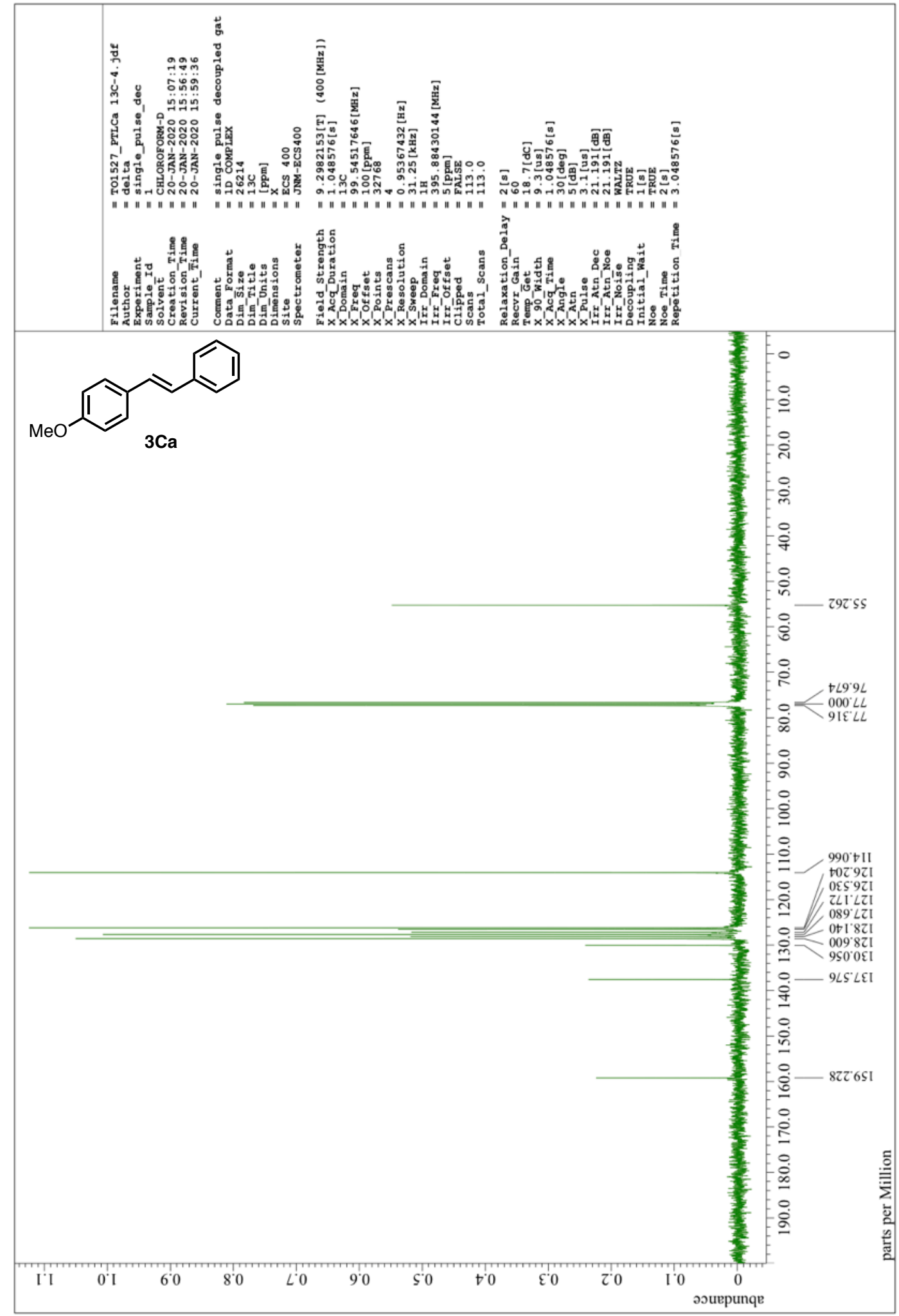


${ }^{1} \mathrm{H}$ NMR of 3Da $\left(400 \mathrm{MHz}, \mathrm{CDCl}_{3}\right)$

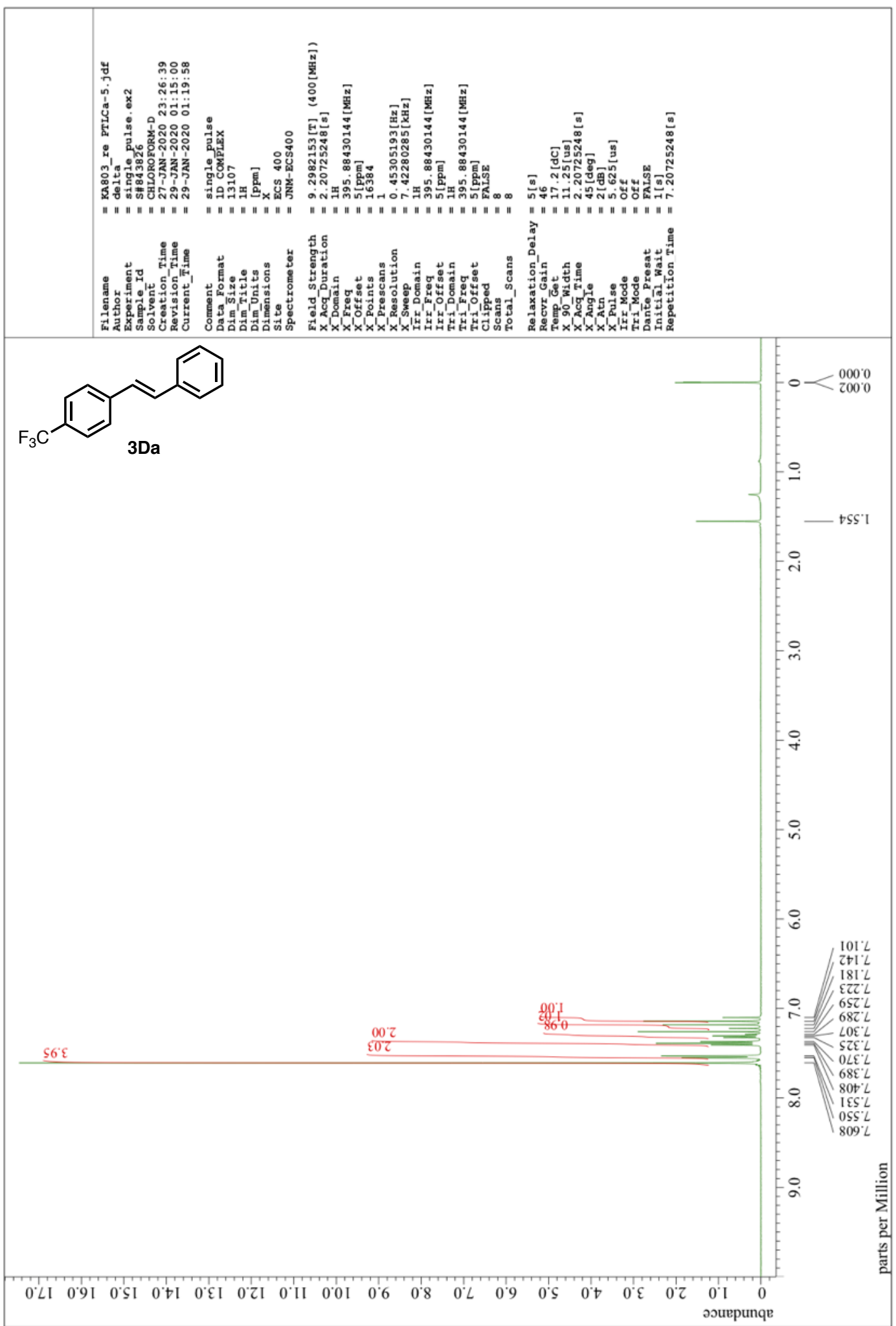


${ }^{13} \mathrm{C}$ NMR of 3Da $\left(101 \mathrm{MHz}, \mathrm{CDCl}_{3}\right)$

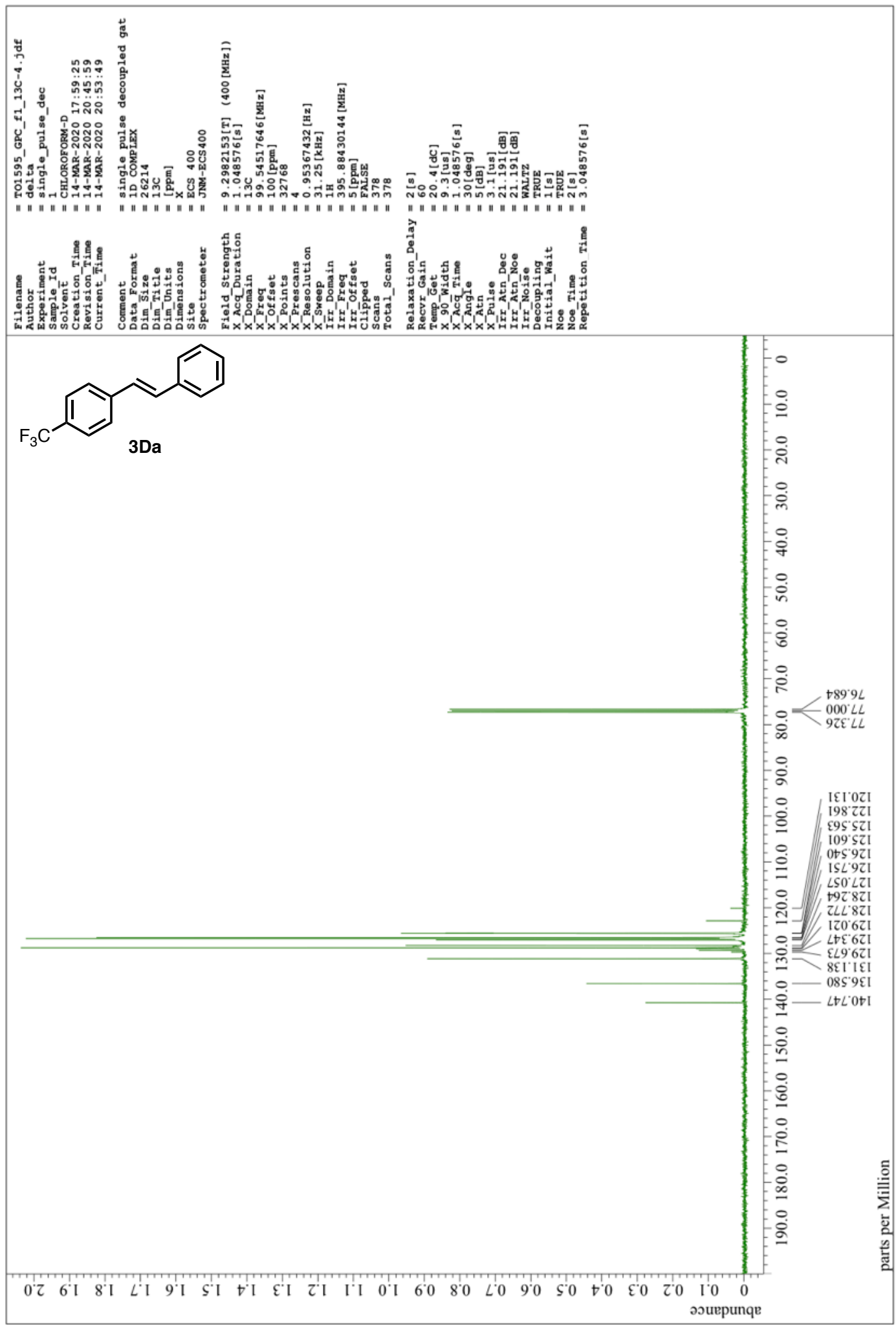


${ }^{1} \mathrm{H}$ NMR of 3Ea $\left(400 \mathrm{MHz}, \mathrm{CDCl}_{3}\right)$

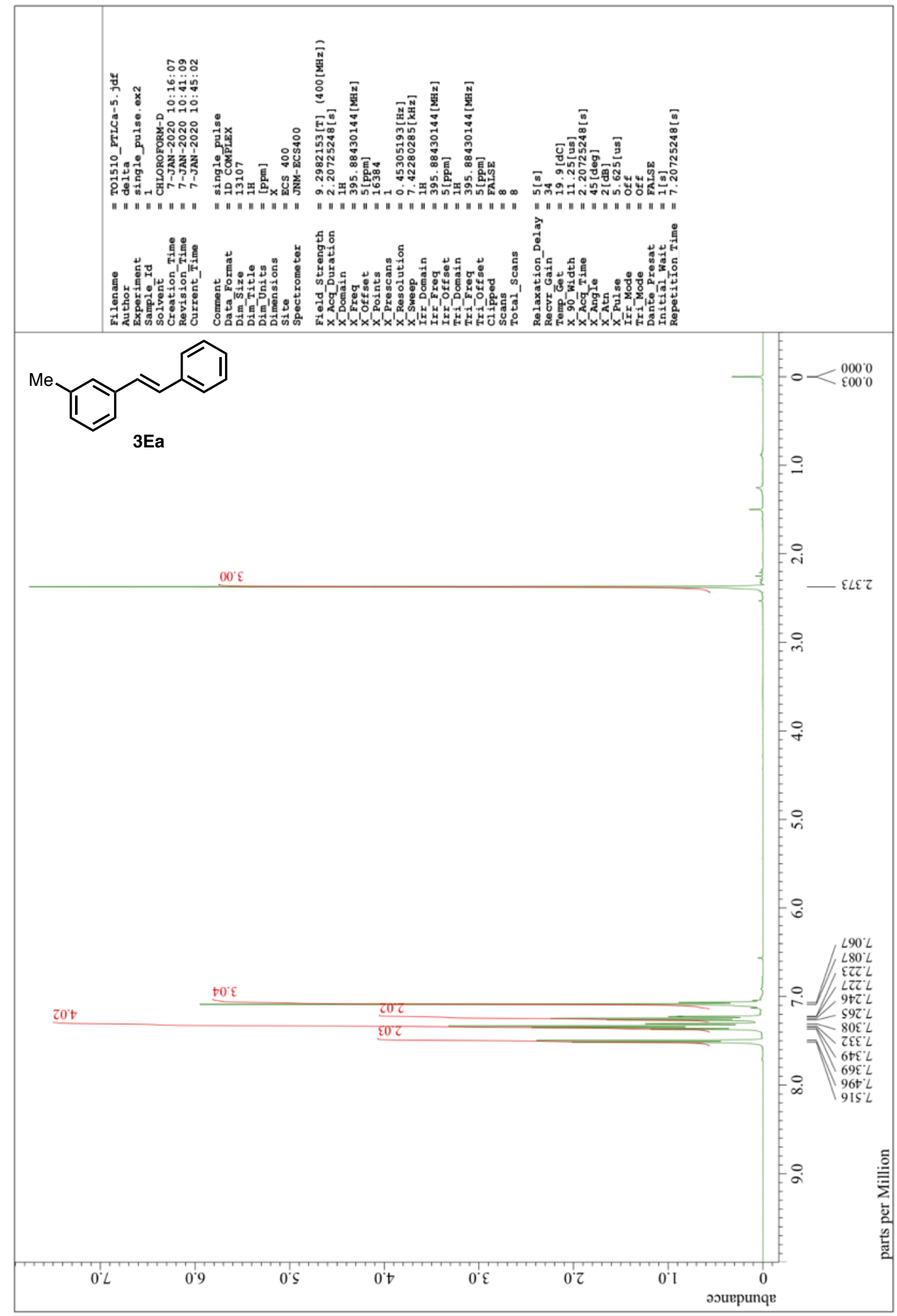


${ }^{13} \mathrm{C}$ NMR of $\mathbf{3 E a}\left(101 \mathrm{MHz}, \mathrm{CDCl}_{3}\right)$

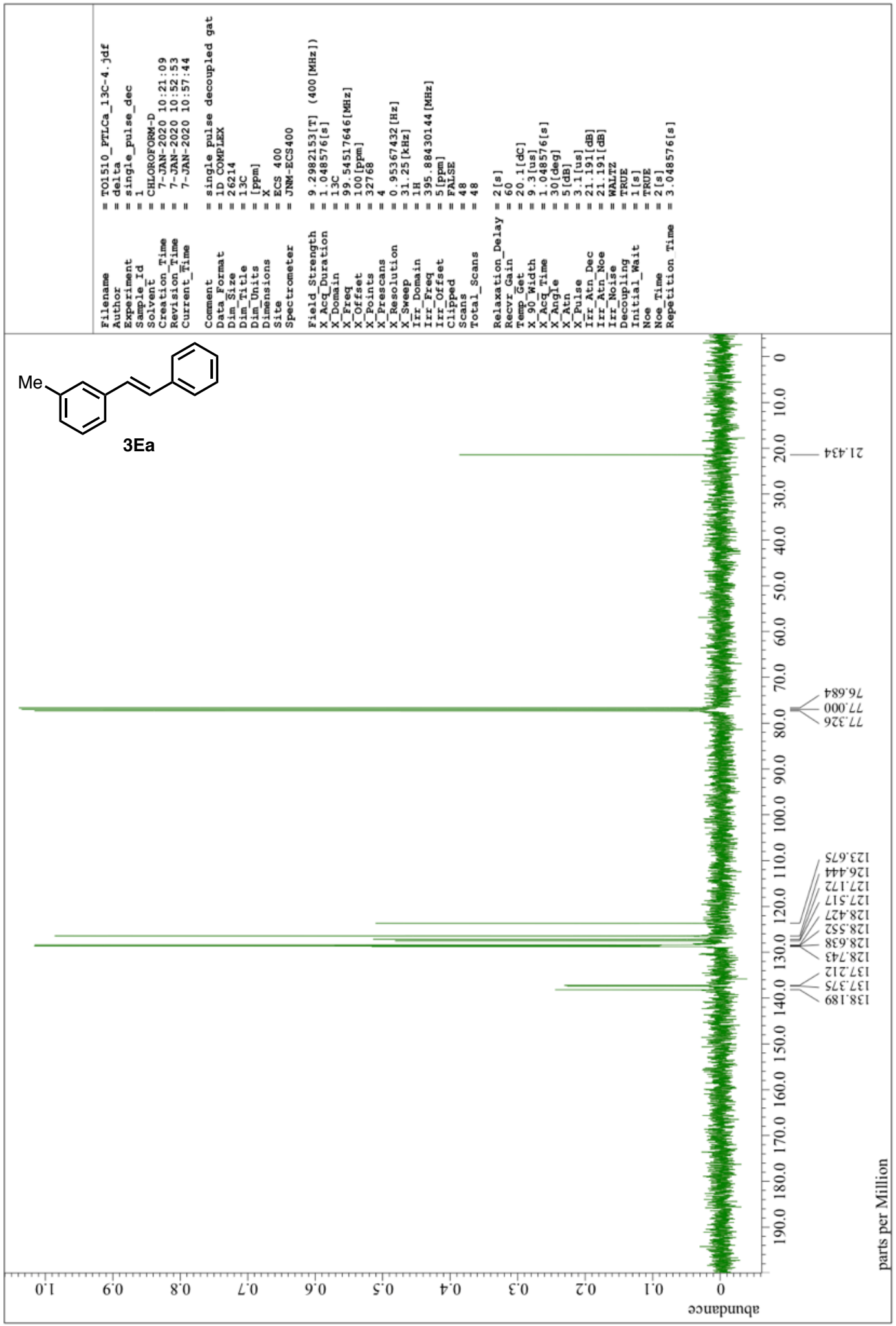


${ }^{1} \mathrm{H} \mathrm{NMR}$ of $\mathbf{3 F a}\left(400 \mathrm{MHz}, \mathrm{CDCl}_{3}\right)$

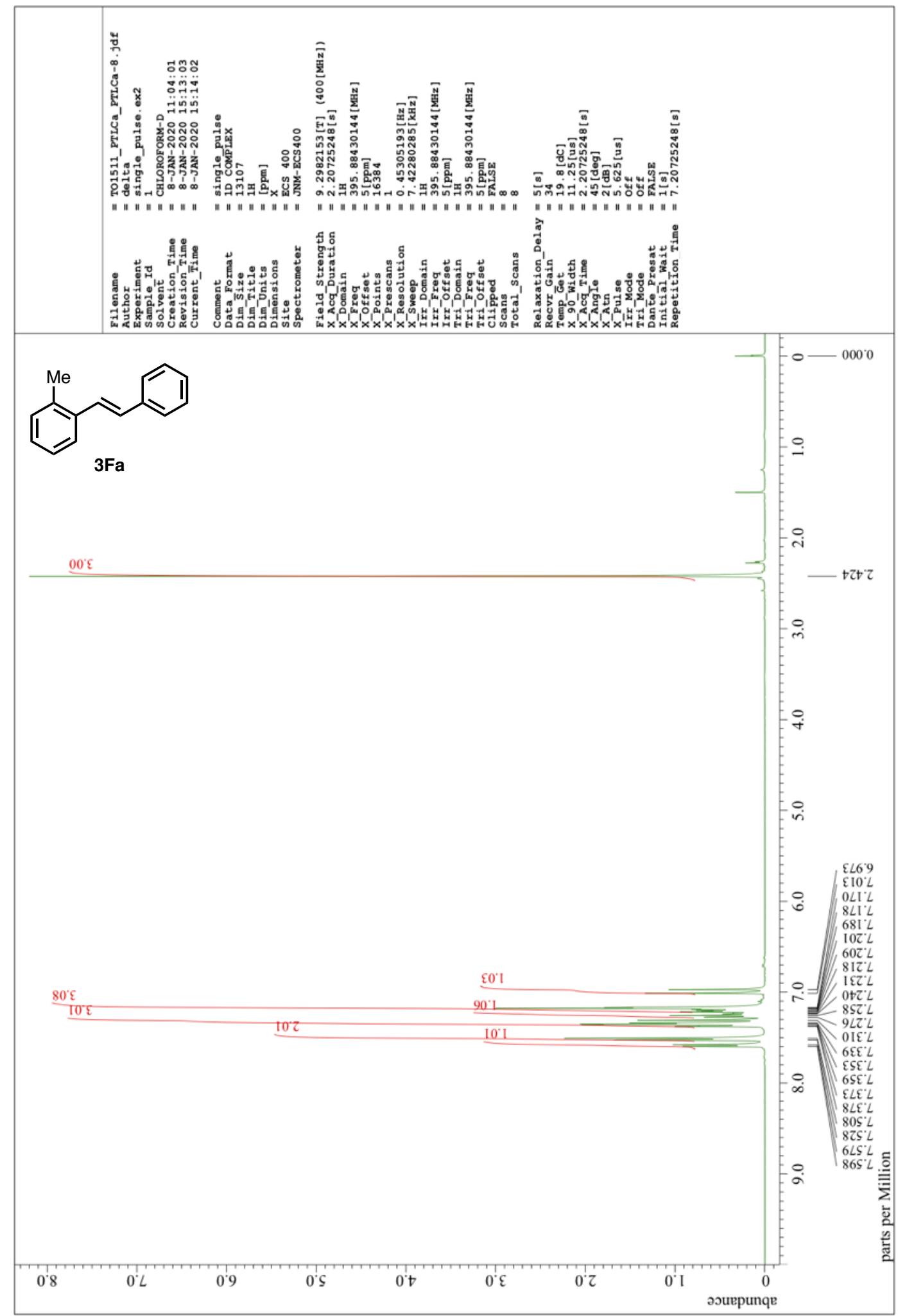


${ }^{13} \mathrm{C} \mathrm{NMR}$ of $\mathbf{3 F a}\left(101 \mathrm{MHz}, \mathrm{CDCl}_{3}\right)$

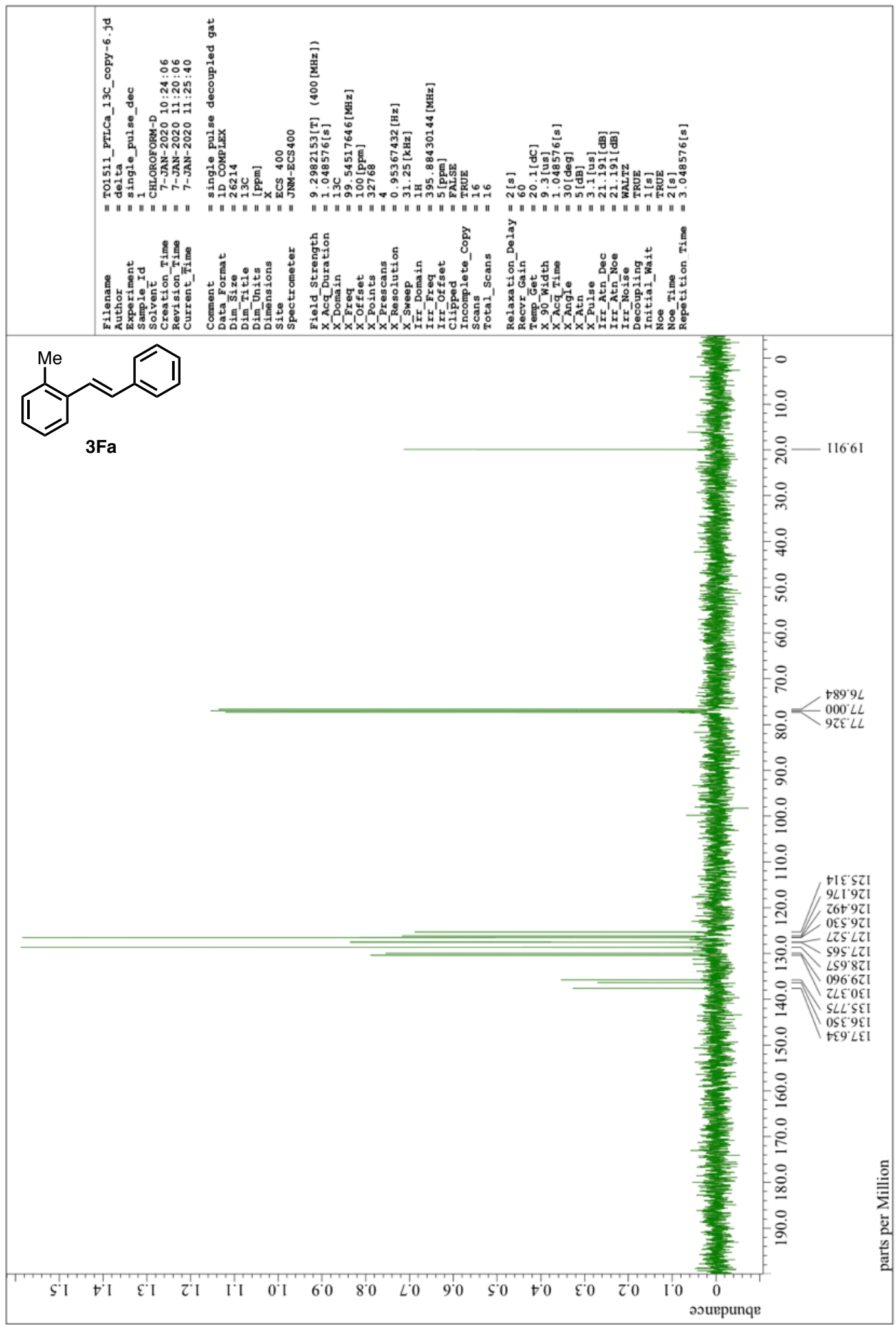


${ }^{1} \mathrm{H}$ NMR of $\mathbf{3 G a}\left(400 \mathrm{MHz}, \mathrm{CDCl}_{3}\right)$

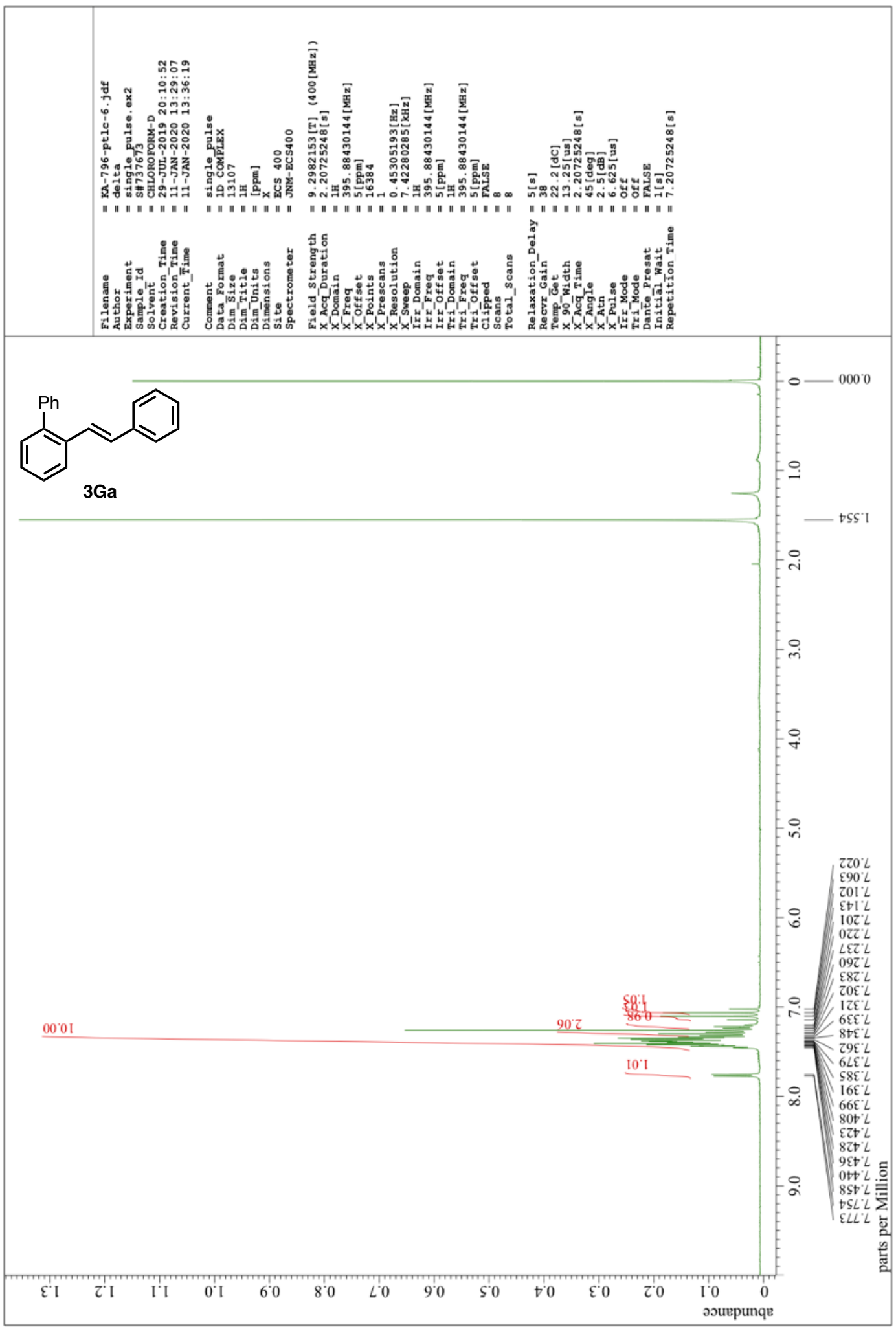


${ }^{13} \mathrm{C}$ NMR of $\mathbf{3 G a}\left(101 \mathrm{MHz}, \mathrm{CDCl}_{3}\right)$

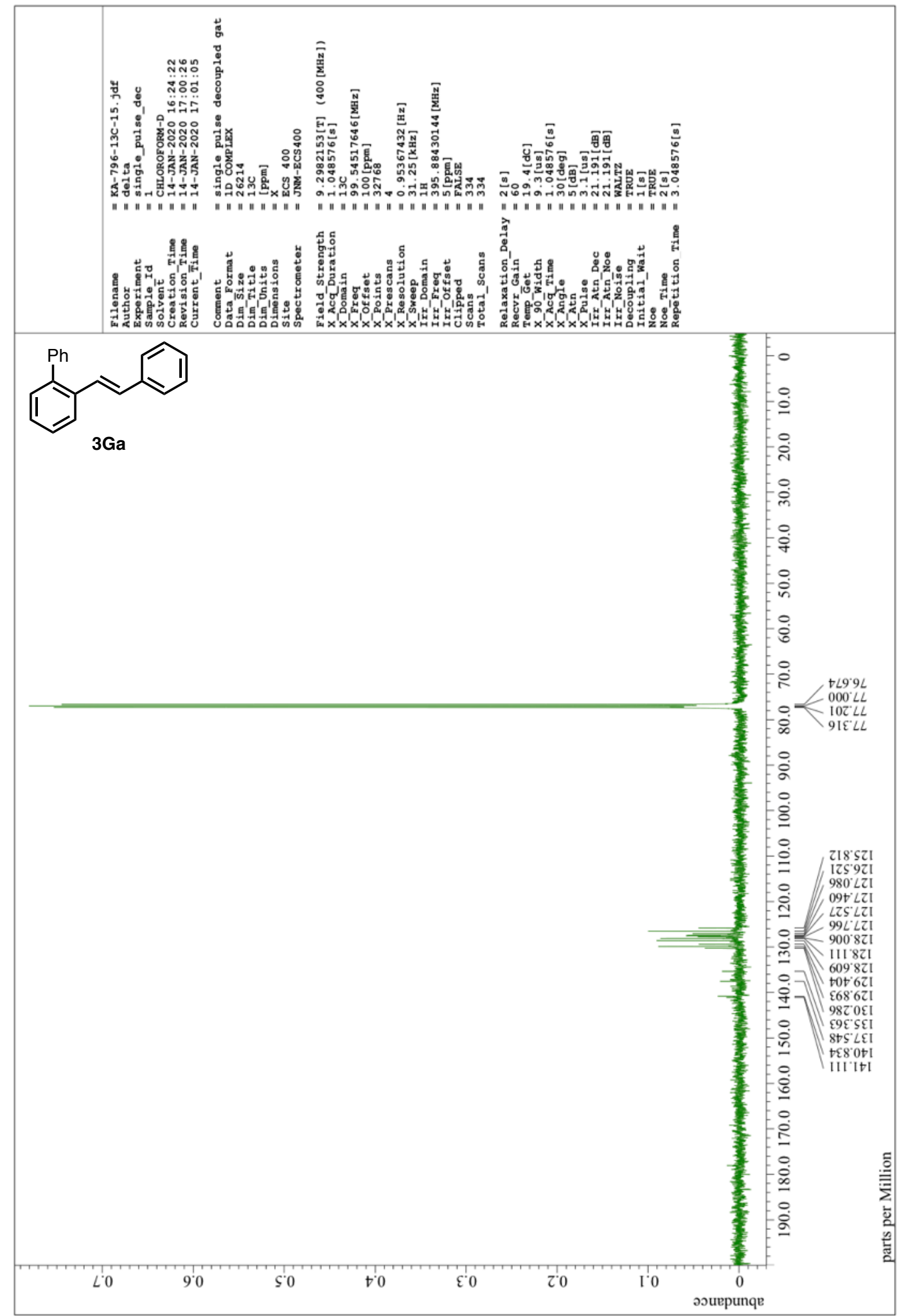


${ }^{1} \mathrm{H}$ NMR of $\mathbf{3 H a}\left(400 \mathrm{MHz}, \mathrm{CDCl}_{3}\right)$

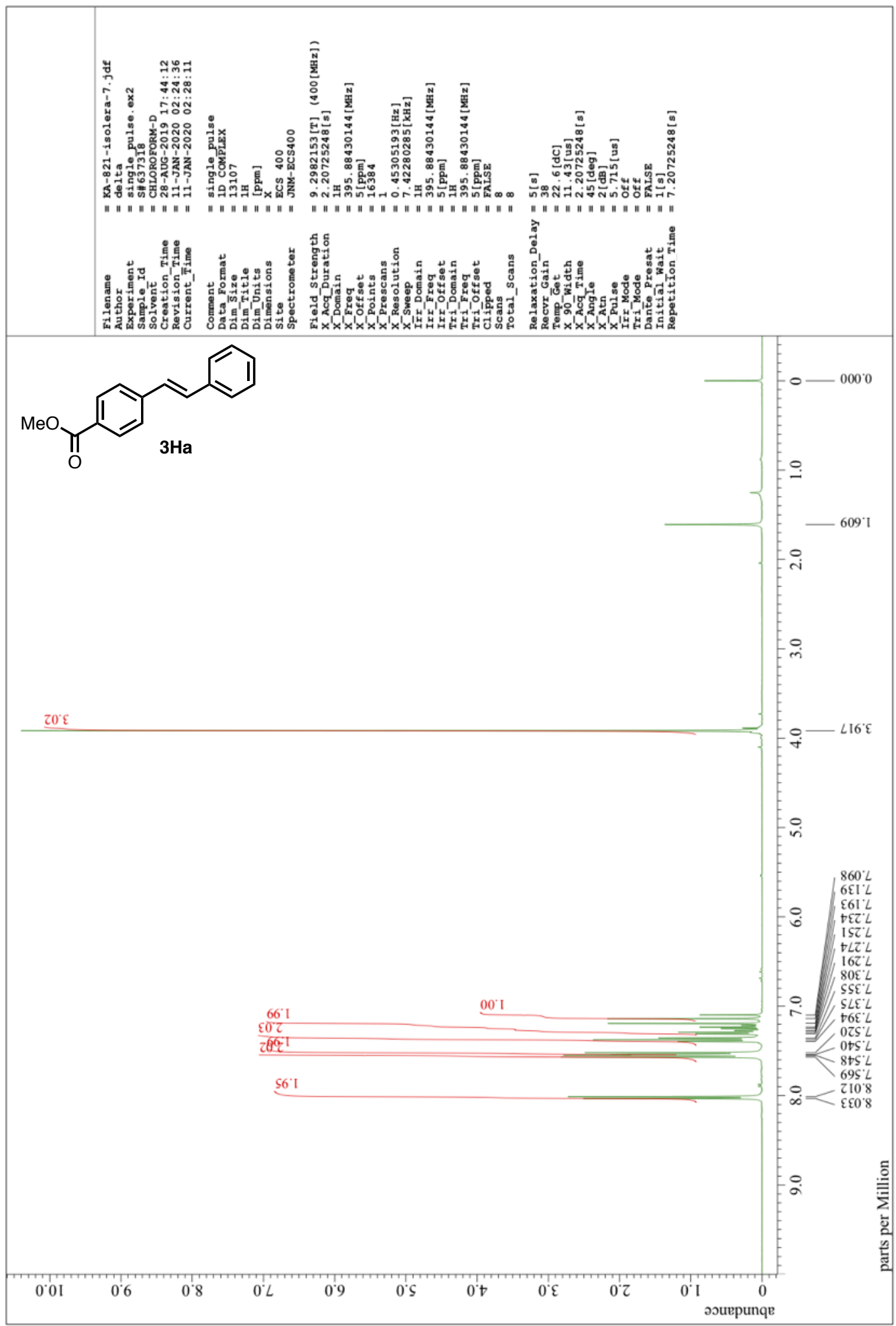


${ }^{13} \mathrm{C}$ NMR of $\mathbf{3 H a}\left(101 \mathrm{MHz}, \mathrm{CDCl}_{3}\right)$

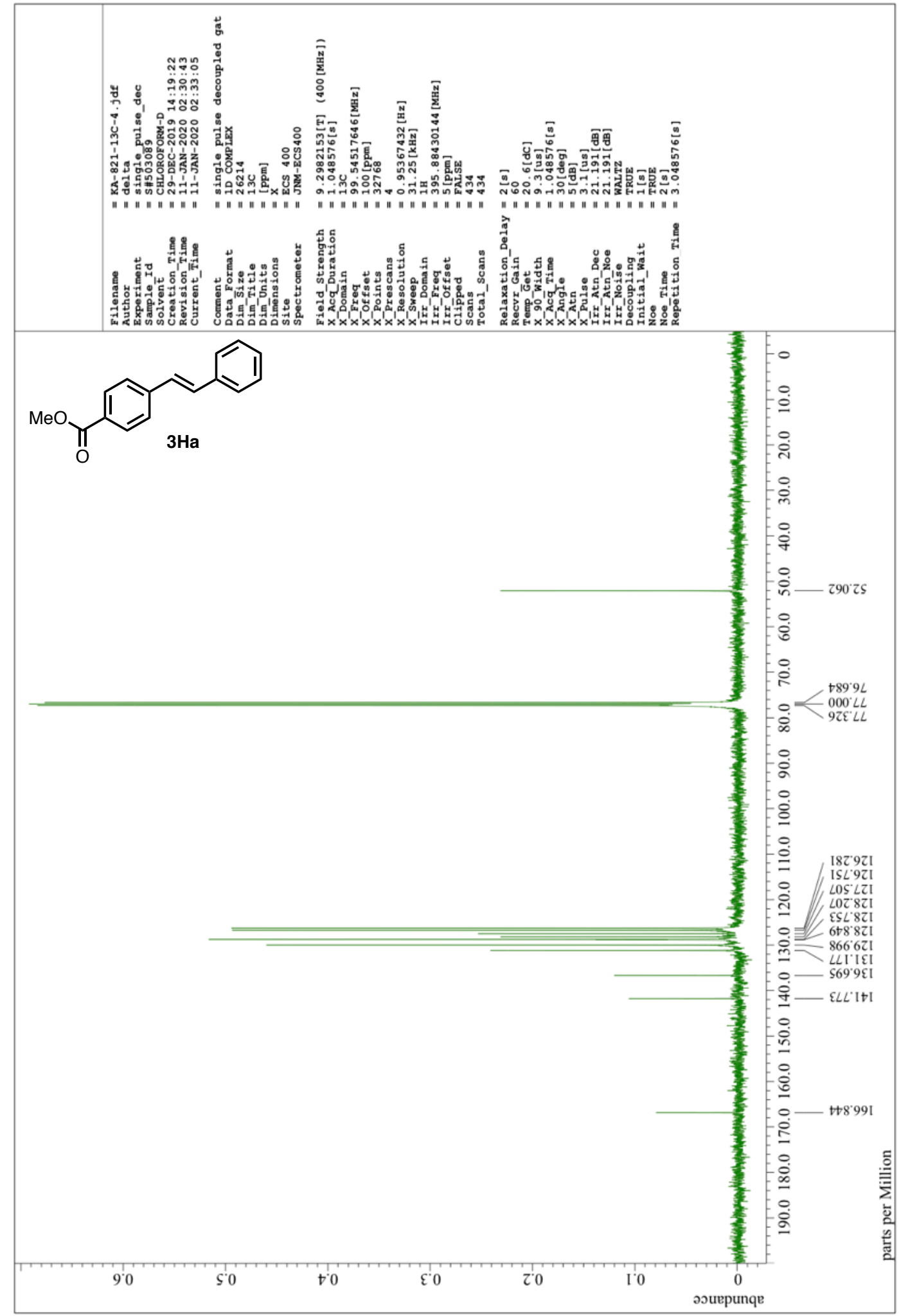


${ }^{1} \mathrm{H}$ NMR of $\mathbf{3 I a}\left(400 \mathrm{MHz}, \mathrm{CDCl}_{3}\right)$

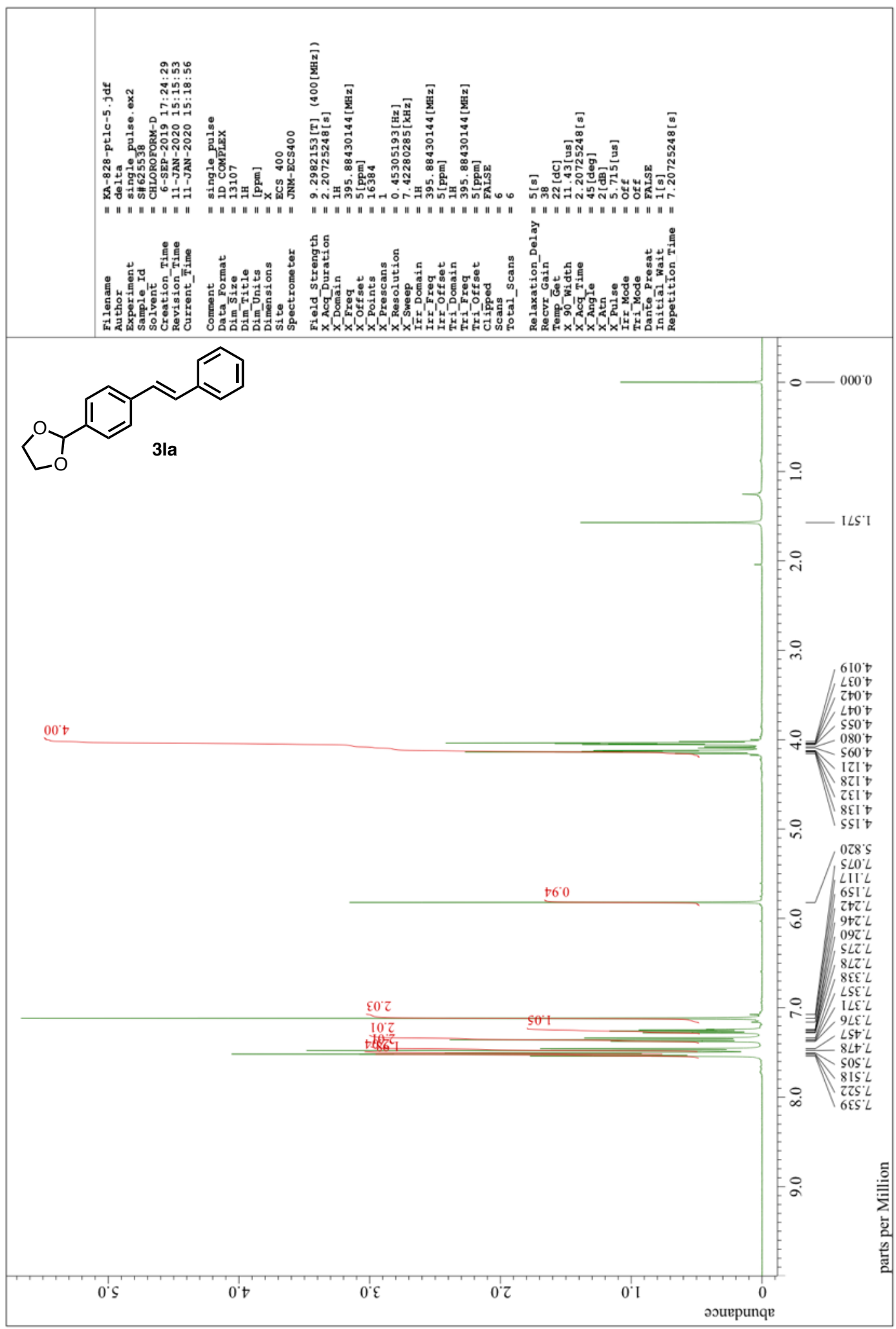


${ }^{13} \mathrm{C}$ NMR of 3Ia $\left(101 \mathrm{MHz}, \mathrm{CDCl}_{3}\right)$

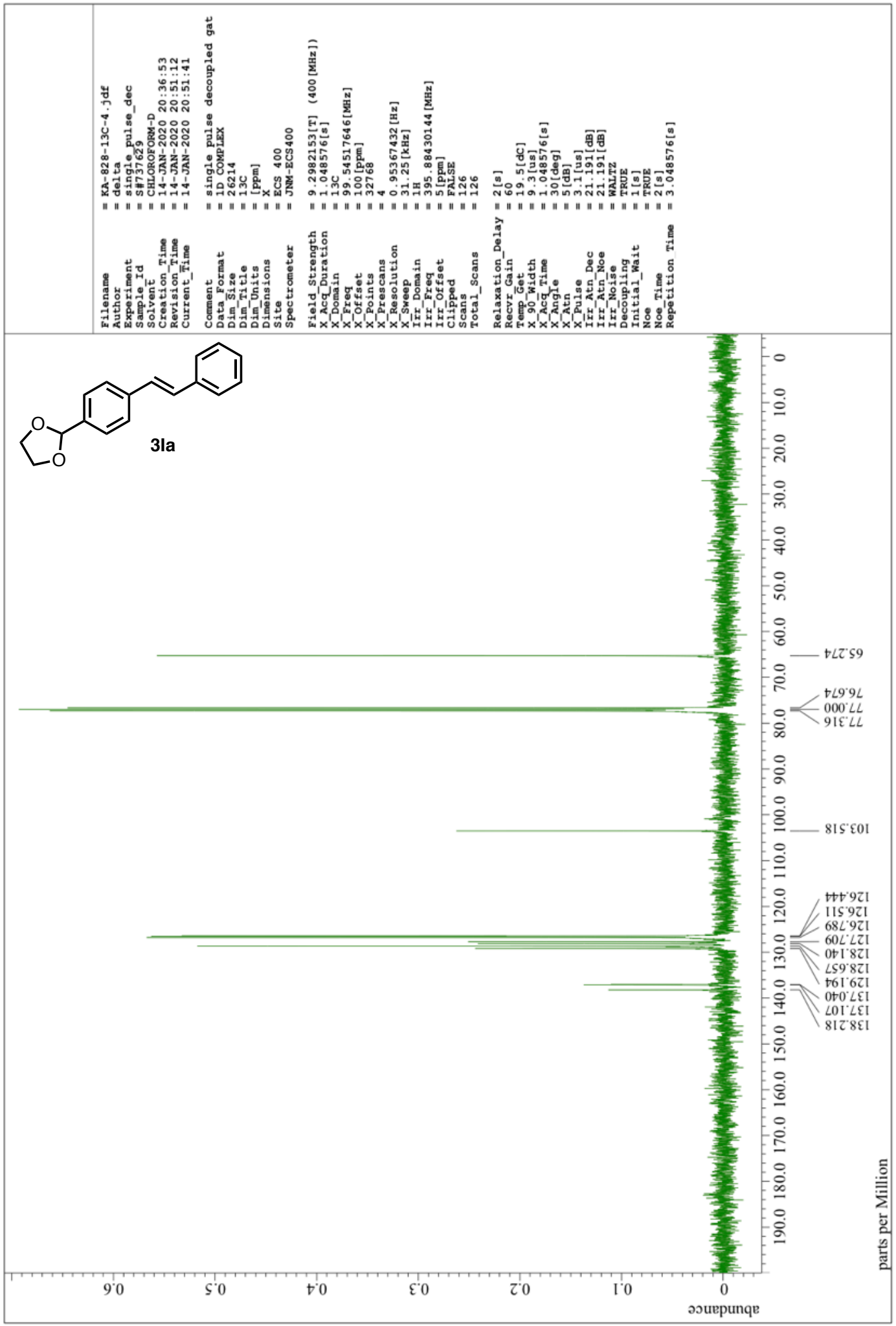


${ }^{1} \mathrm{H}$ NMR of $\mathbf{3 J a}\left(400 \mathrm{MHz}, \mathrm{CDCl}_{3}\right)$

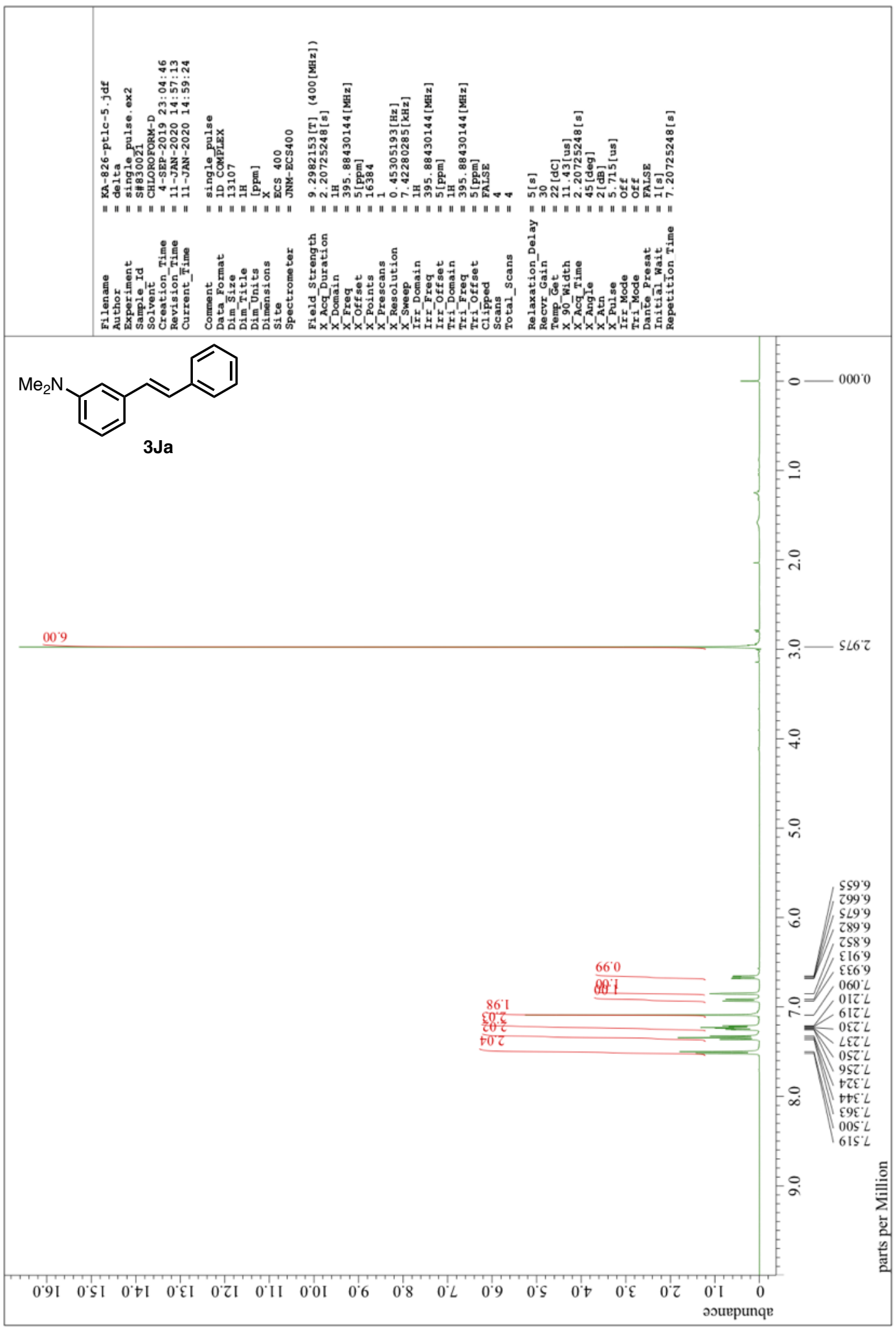


${ }^{13} \mathrm{C}$ NMR of 3Ja $\left(101 \mathrm{MHz}, \mathrm{CDCl}_{3}\right)$

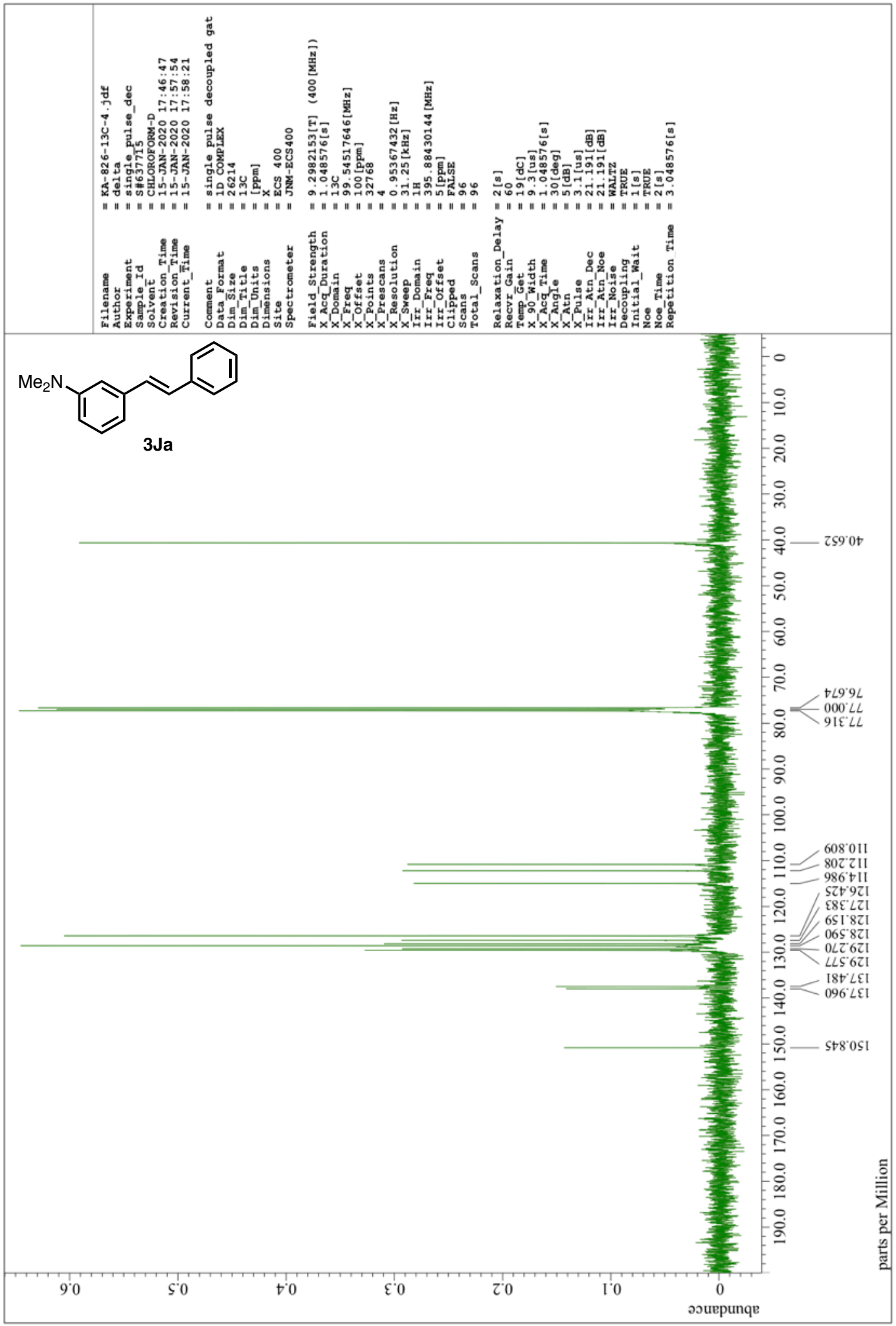


${ }^{1} \mathrm{H}$ NMR of $\mathbf{3 K a}\left(400 \mathrm{MHz}, \mathrm{CDCl}_{3}\right)$

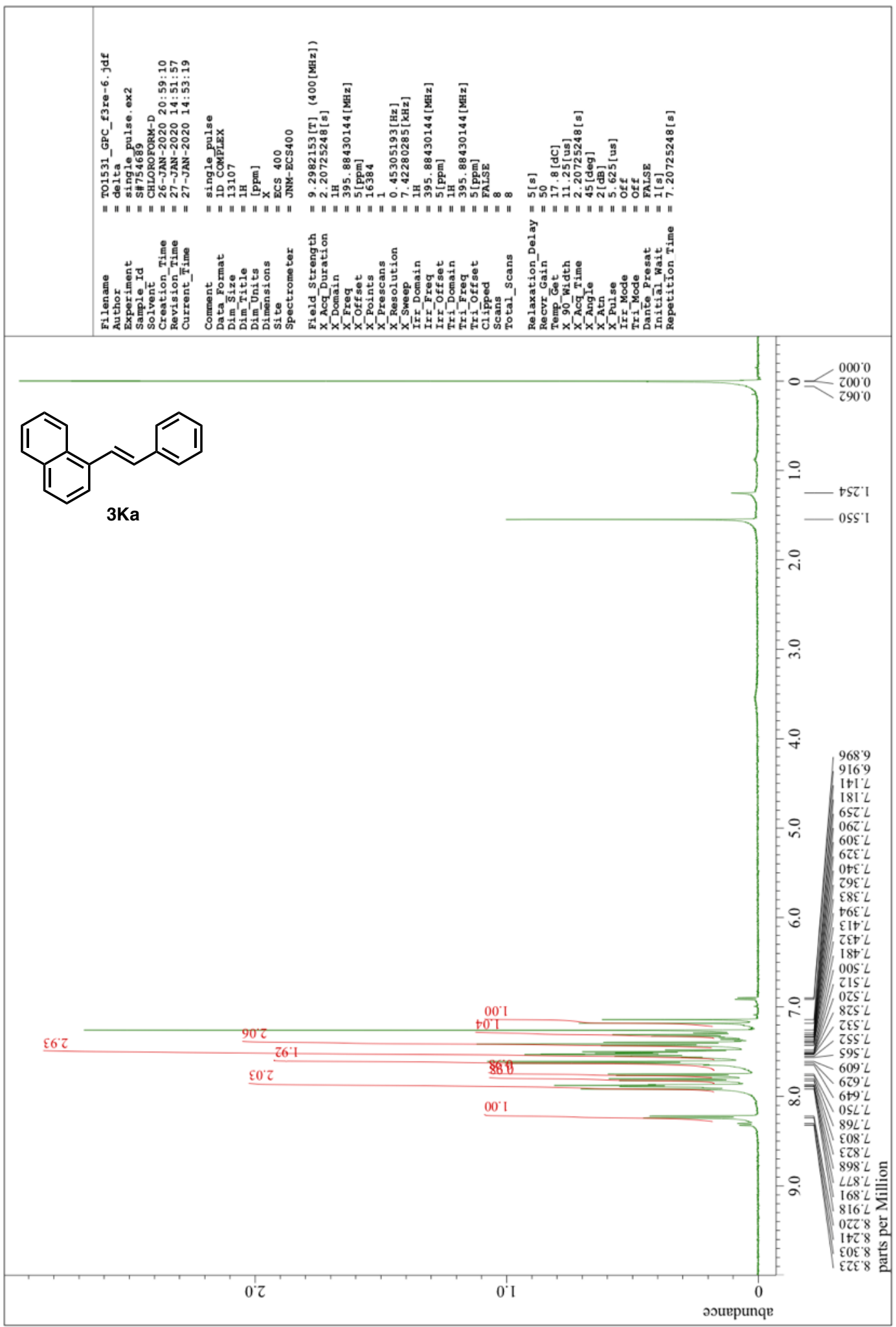


${ }^{13} \mathrm{C}$ NMR of $\mathbf{3 K a}\left(101 \mathrm{MHz}, \mathrm{CDCl}_{3}\right)$

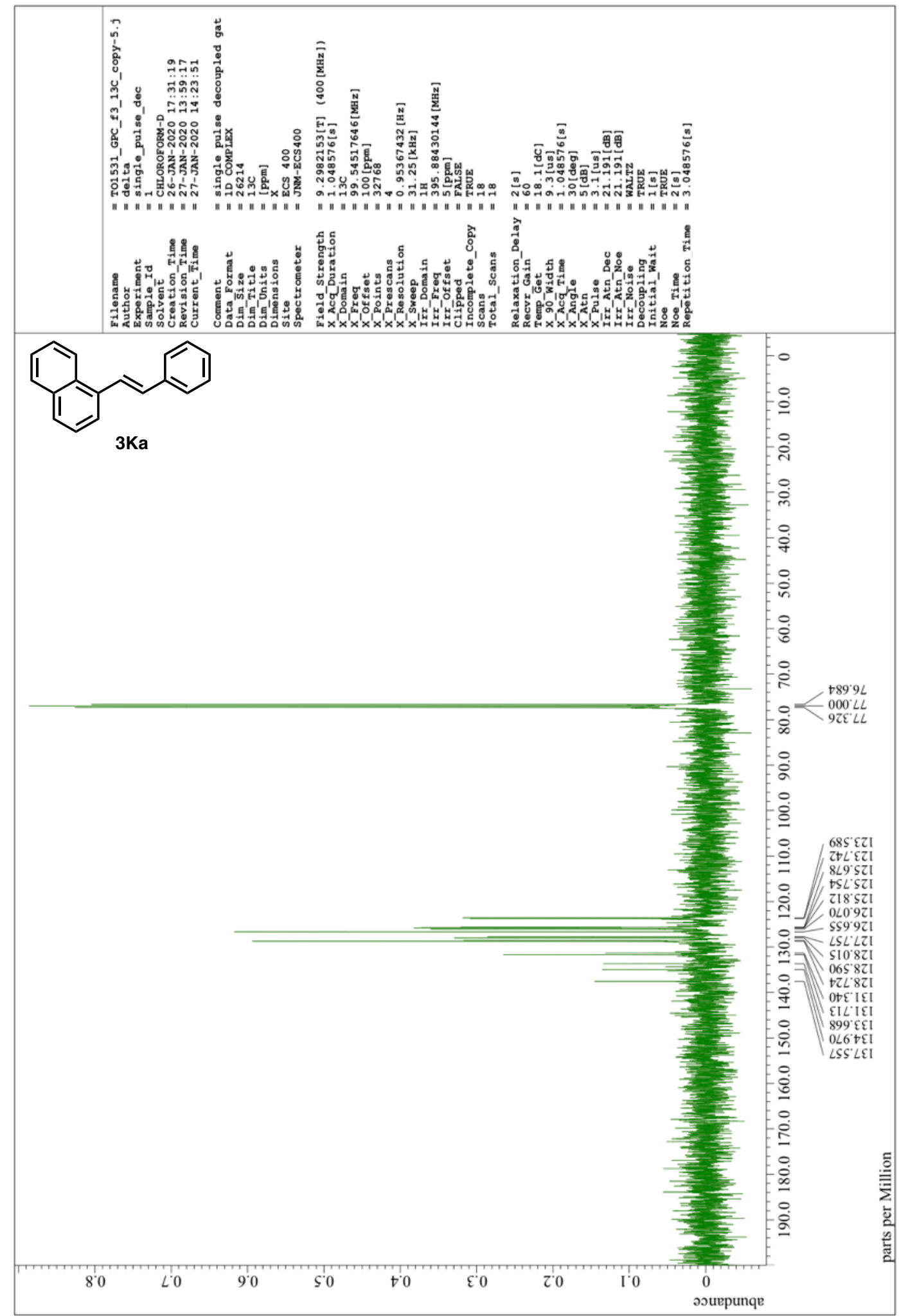


${ }^{1} \mathrm{H}$ NMR of $\mathbf{3 L a}\left(400 \mathrm{MHz}, \mathrm{CDCl}_{3}\right)$

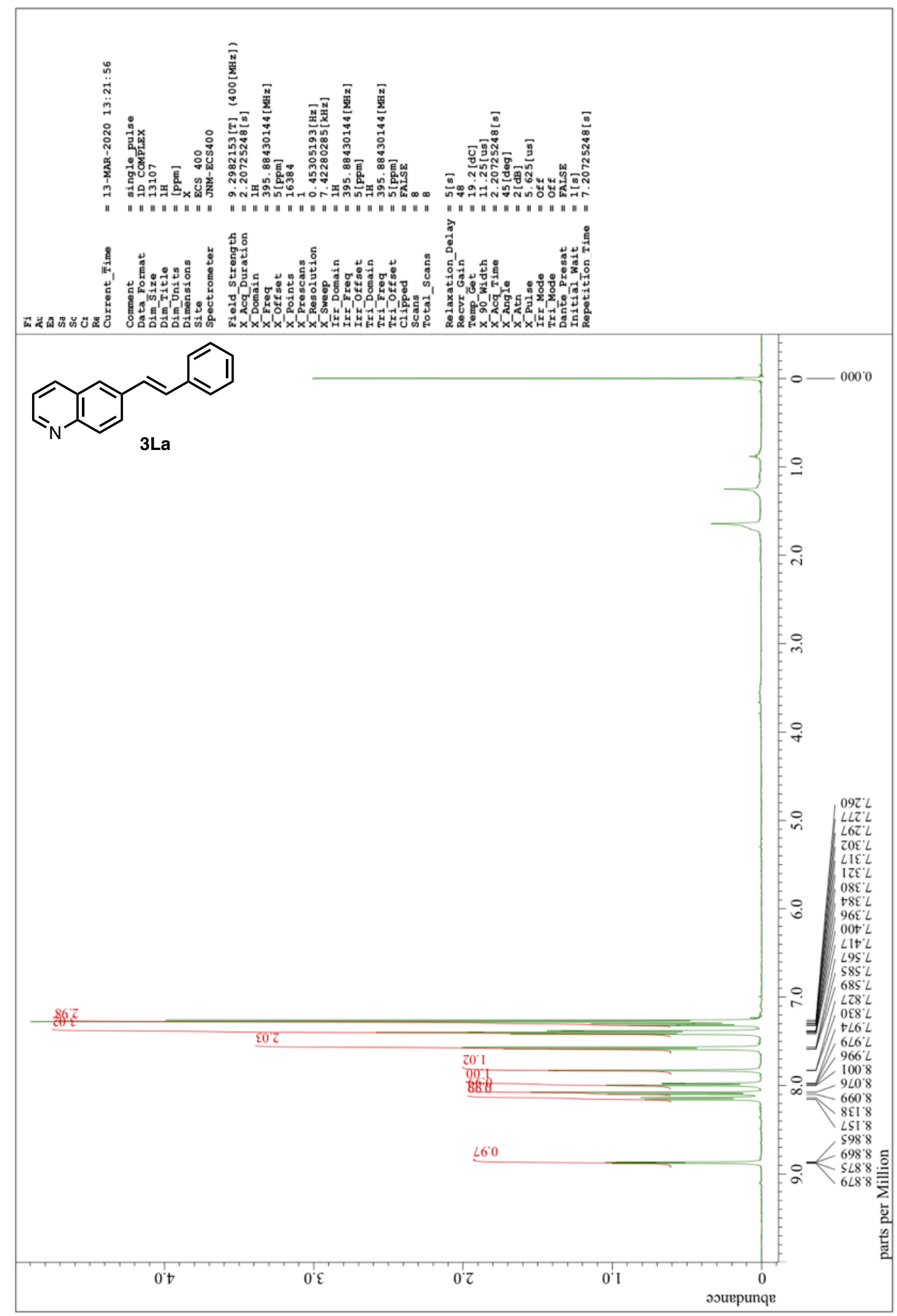


${ }^{13} \mathrm{C}$ NMR of $\mathbf{3} \mathbf{L a}\left(101 \mathrm{MHz}, \mathrm{CDCl}_{3}\right)$

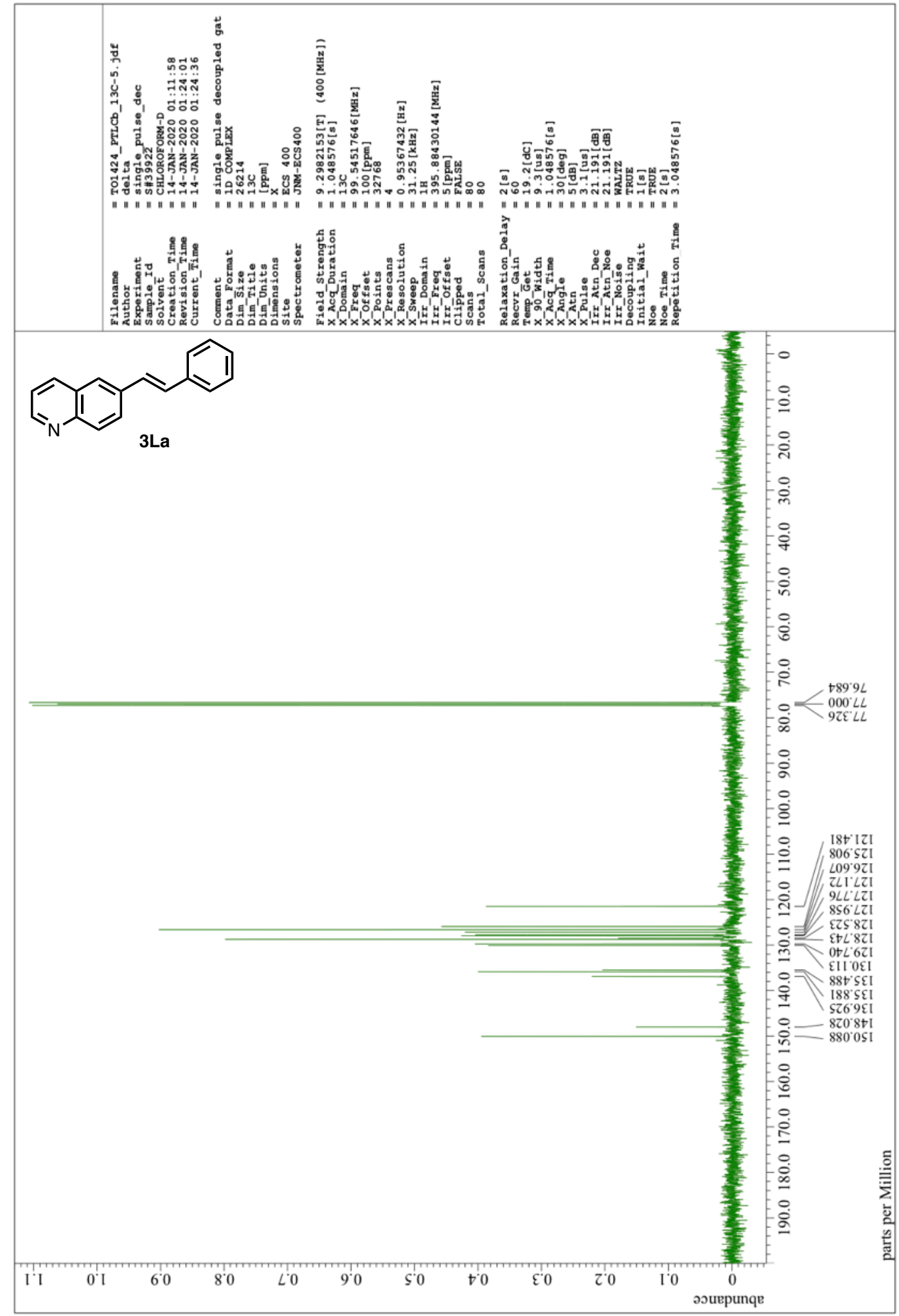


${ }^{1} \mathrm{H} \mathrm{NMR}$ of $\mathbf{3 M a}\left(400 \mathrm{MHz}, \mathrm{CDCl}_{3}\right)$

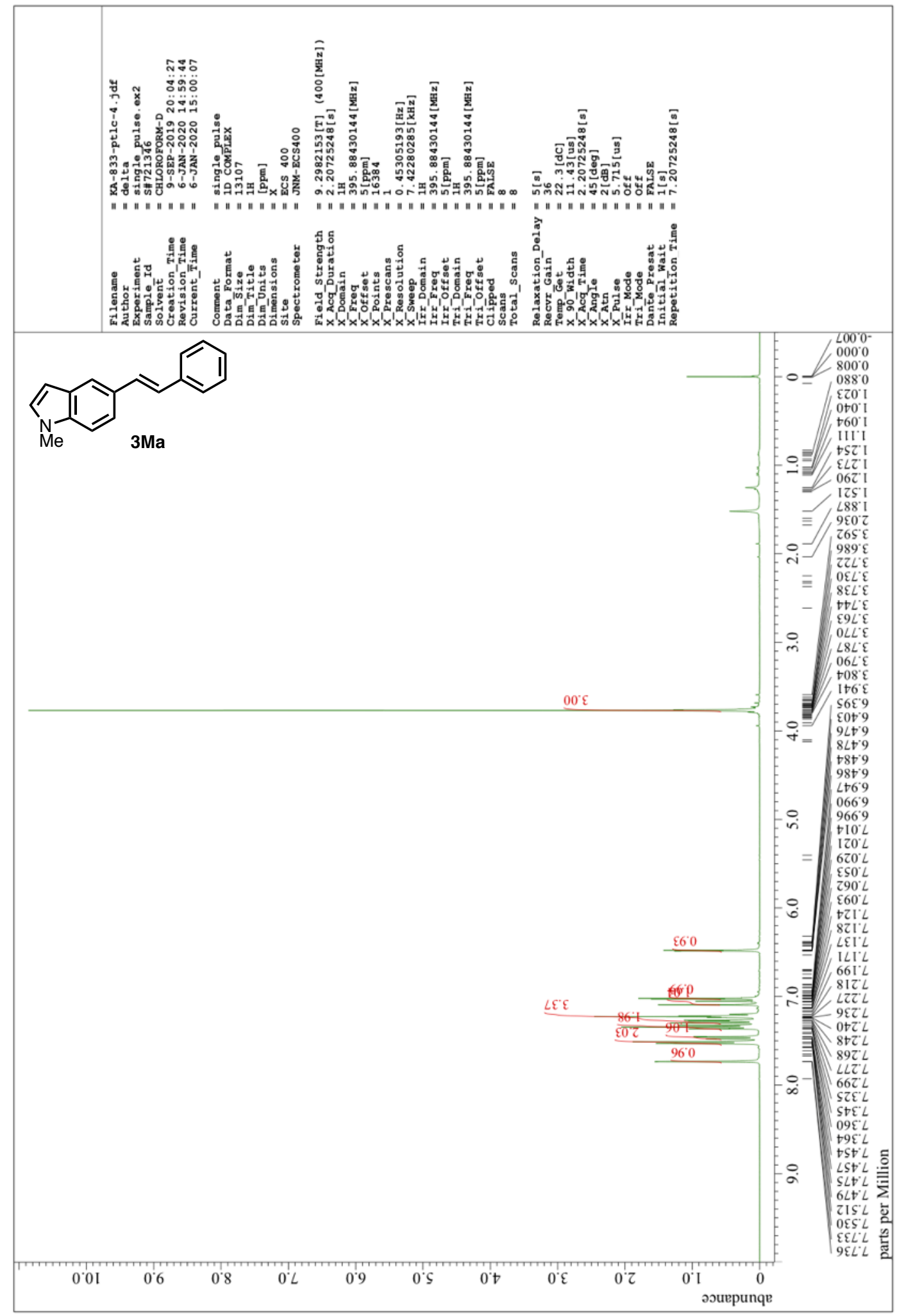


${ }^{13} \mathrm{C}$ NMR of $\mathbf{3 M a}\left(101 \mathrm{MHz}, \mathrm{CDCl}_{3}\right)$

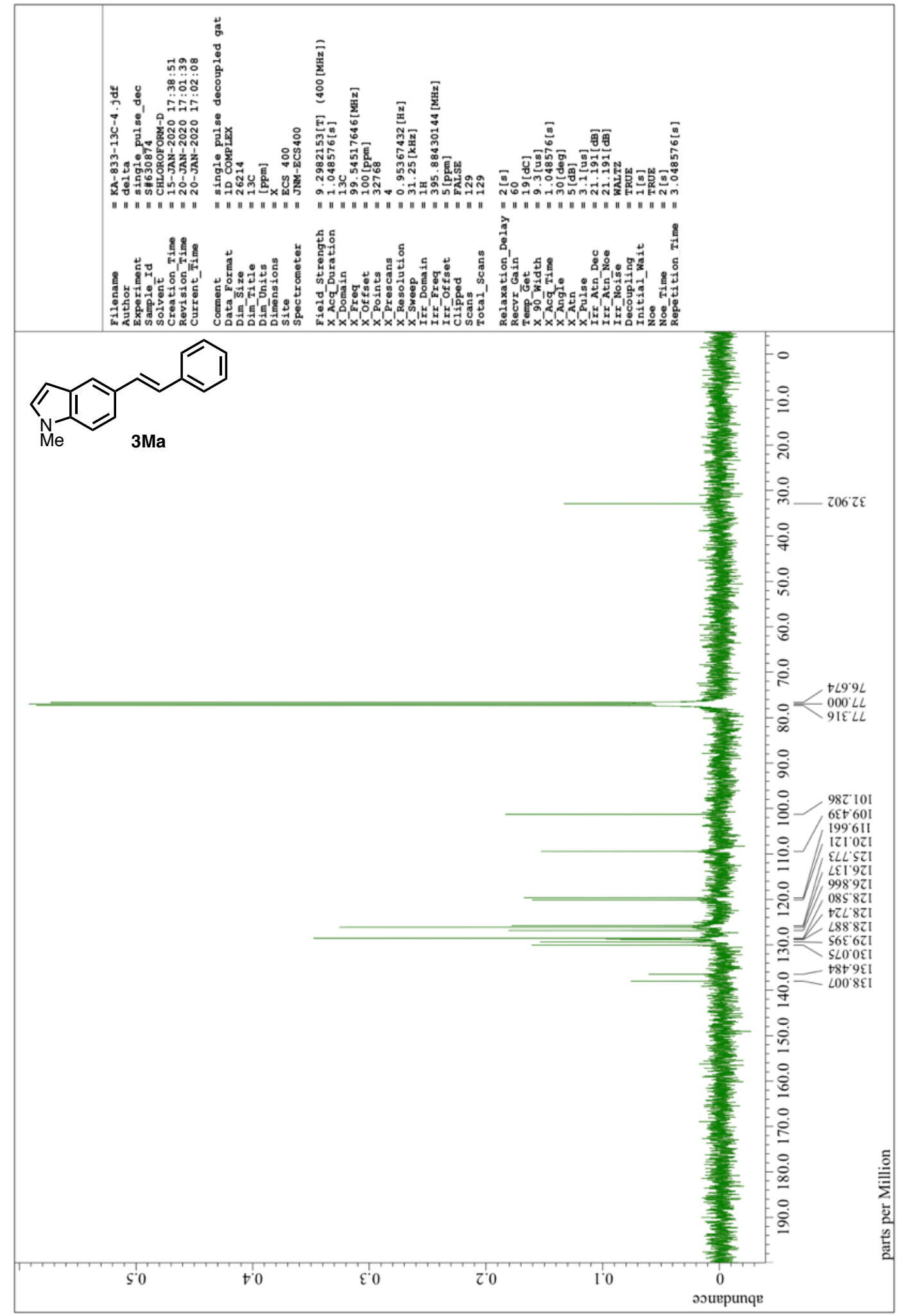


${ }^{1} \mathrm{H}$ NMR of $\mathbf{3 N a}\left(400 \mathrm{MHz}, \mathrm{CDCl}_{3}\right)$

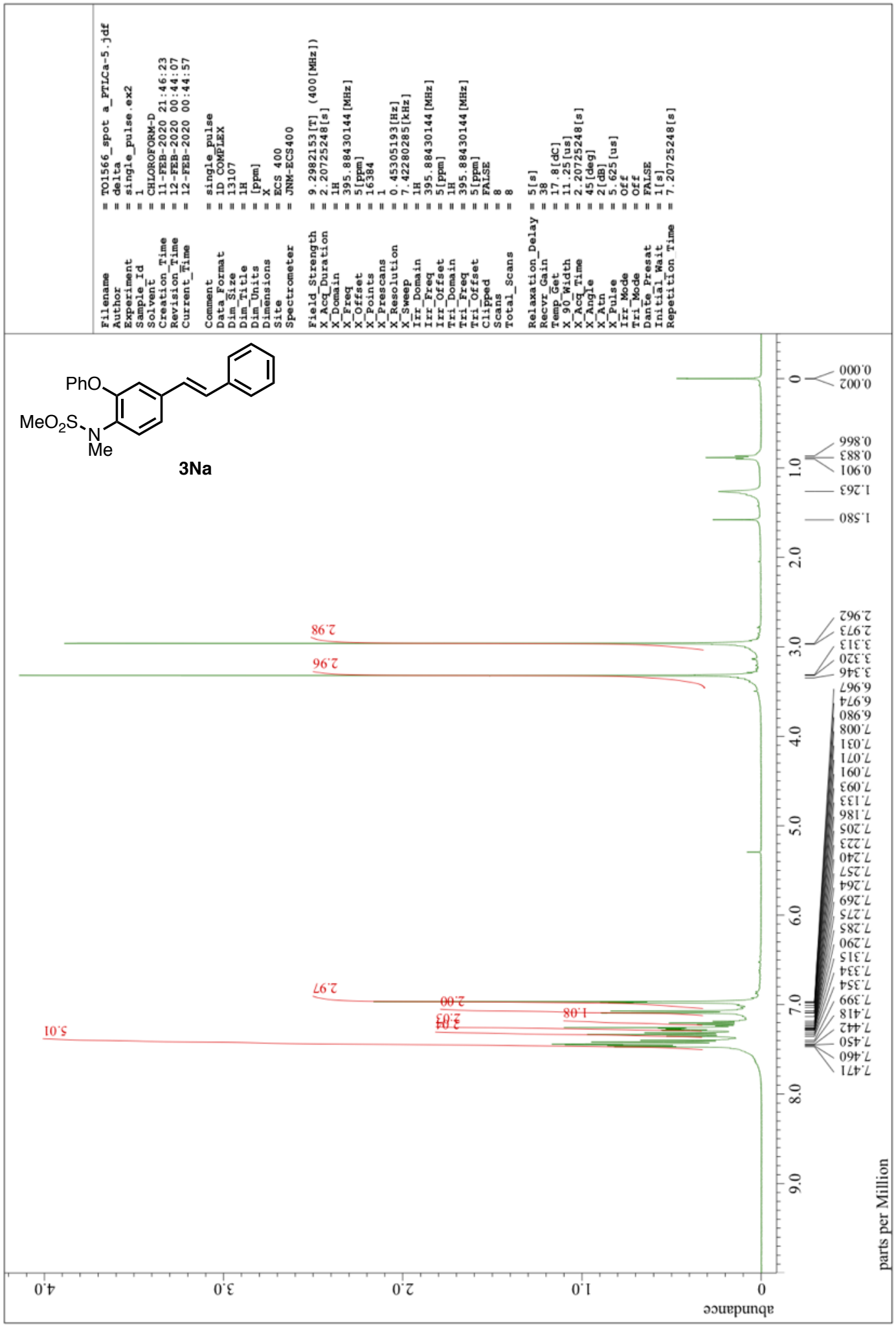


${ }^{13} \mathrm{C}$ NMR of $\mathbf{3 N a}\left(101 \mathrm{MHz}, \mathrm{CDCl}_{3}\right)$

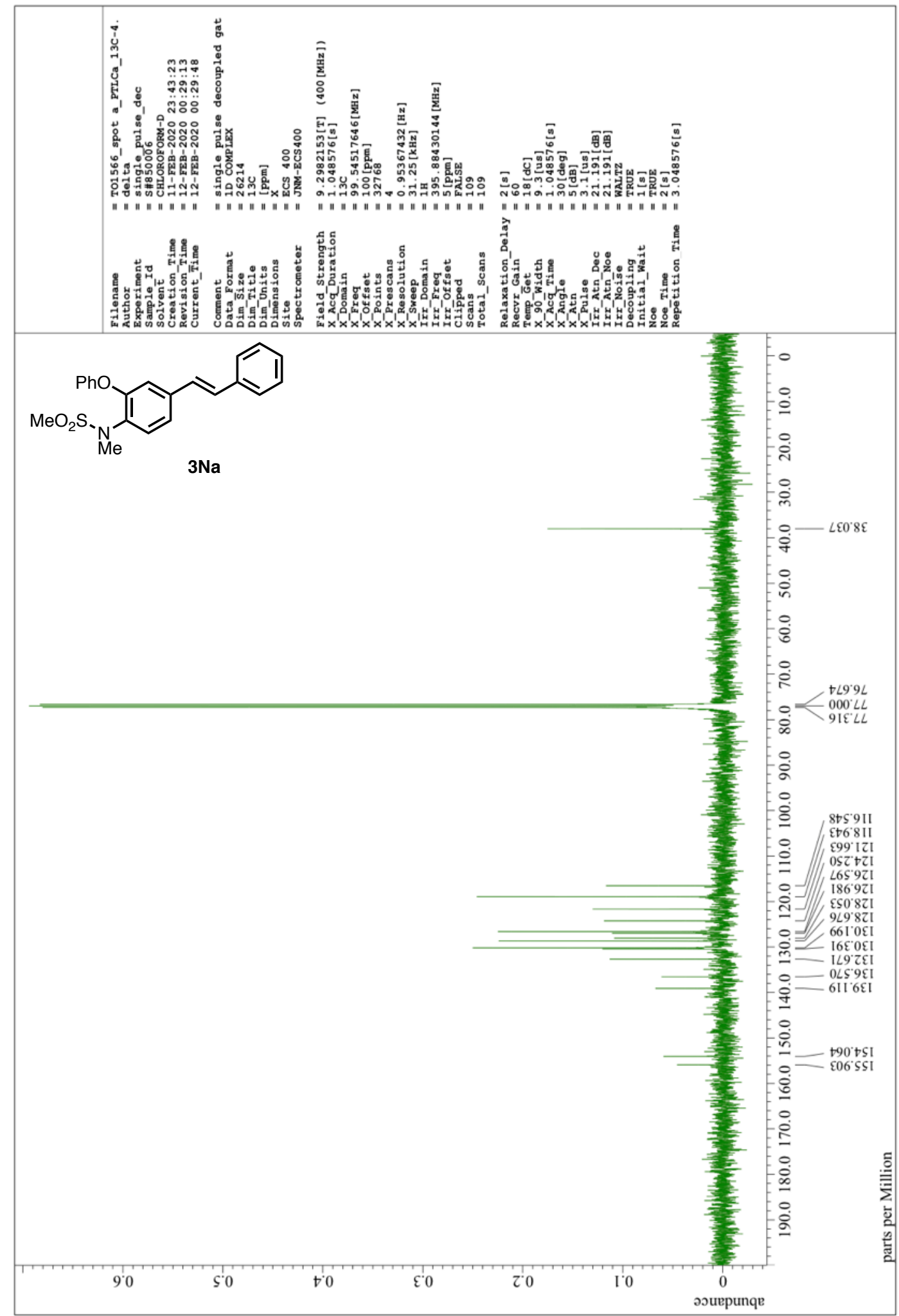


${ }^{1} \mathrm{H}$ NMR of $\mathbf{3 A b}\left(400 \mathrm{MHz}, \mathrm{CDCl}_{3}\right)$

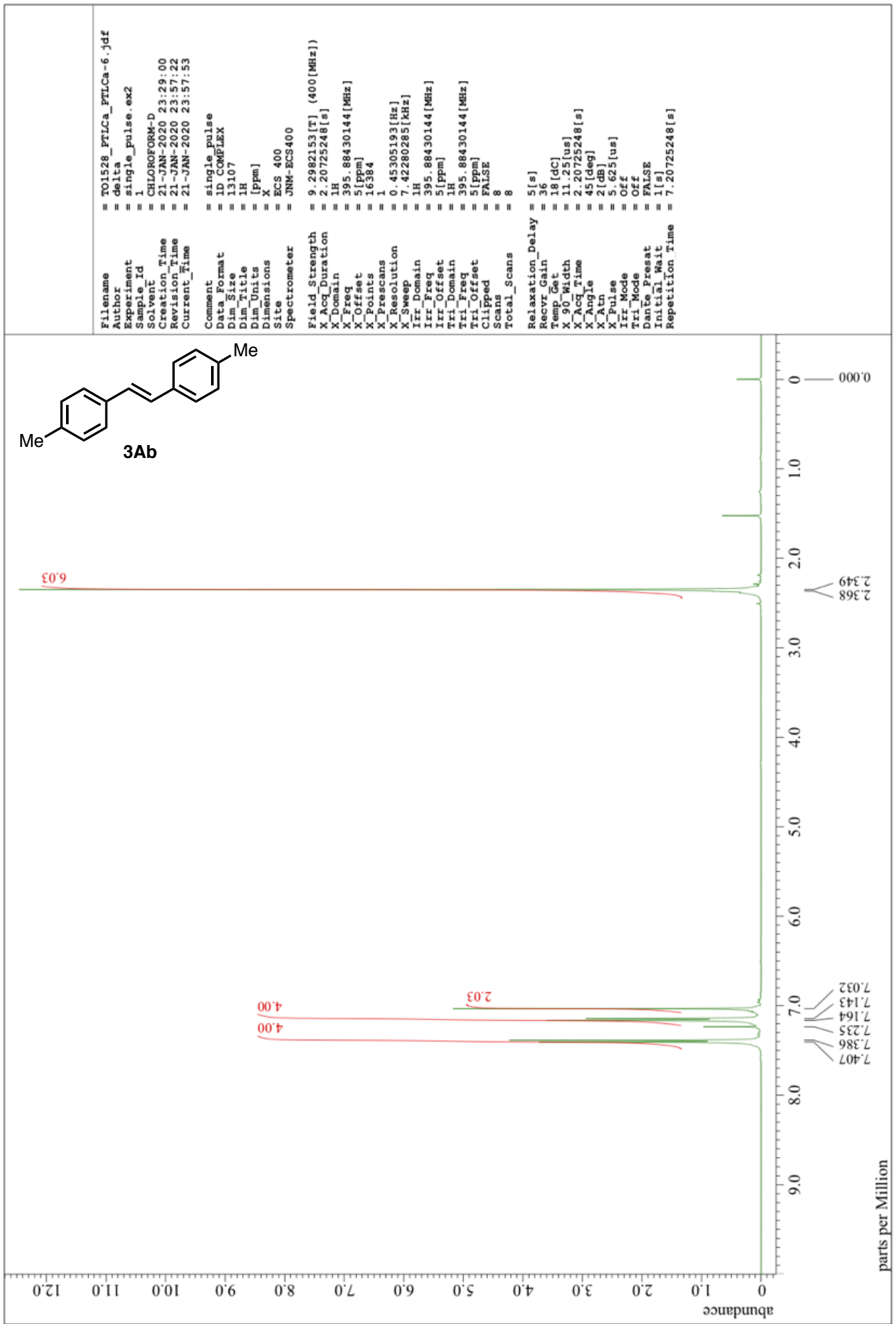


${ }^{13} \mathrm{C}$ NMR of $\mathbf{3 A b}\left(101 \mathrm{MHz}, \mathrm{CDCl}_{3}\right)$

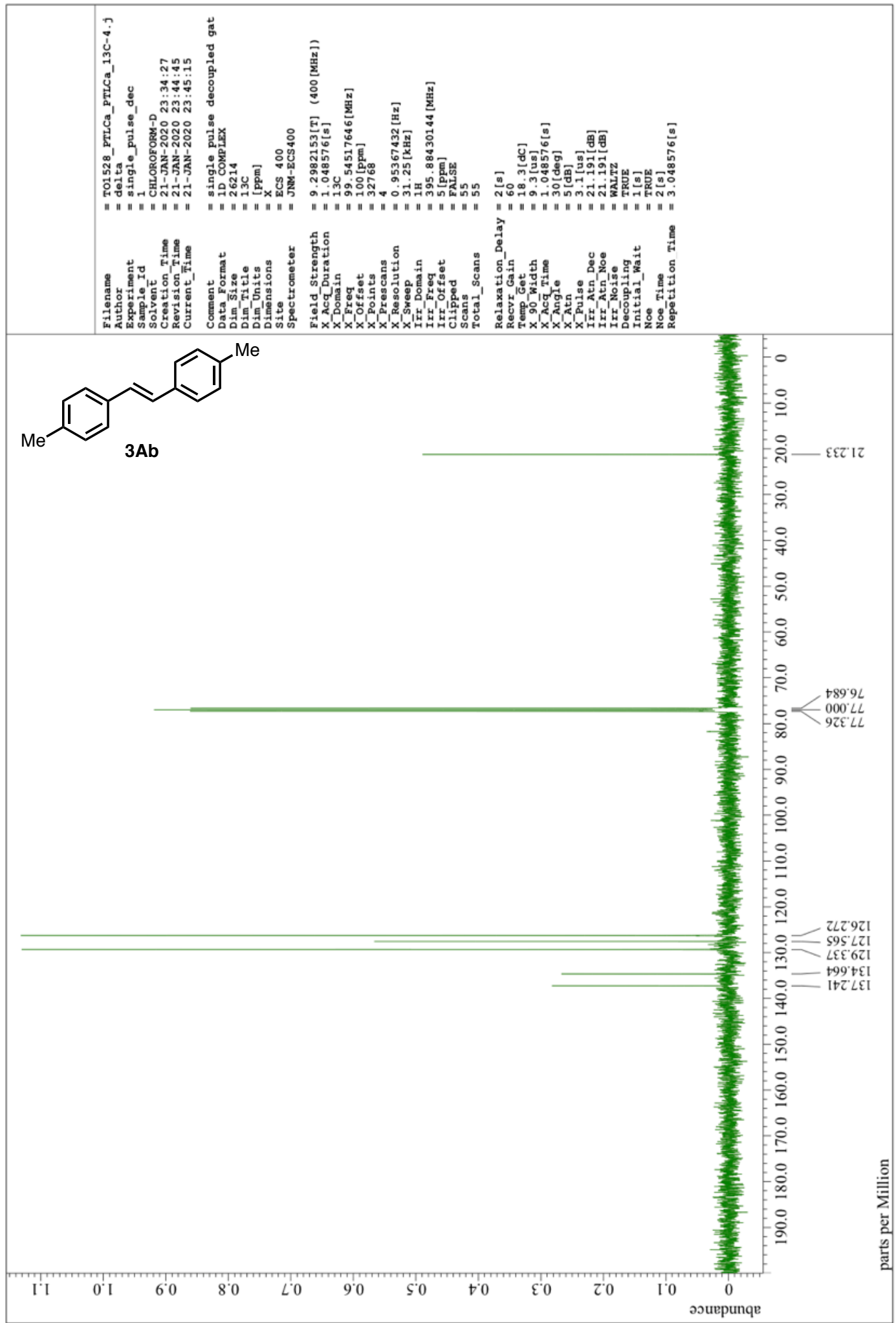


${ }^{1} \mathrm{H}$ NMR of $3 \mathrm{Ac}\left(400 \mathrm{MHz}, \mathrm{CDCl}_{3}\right)$

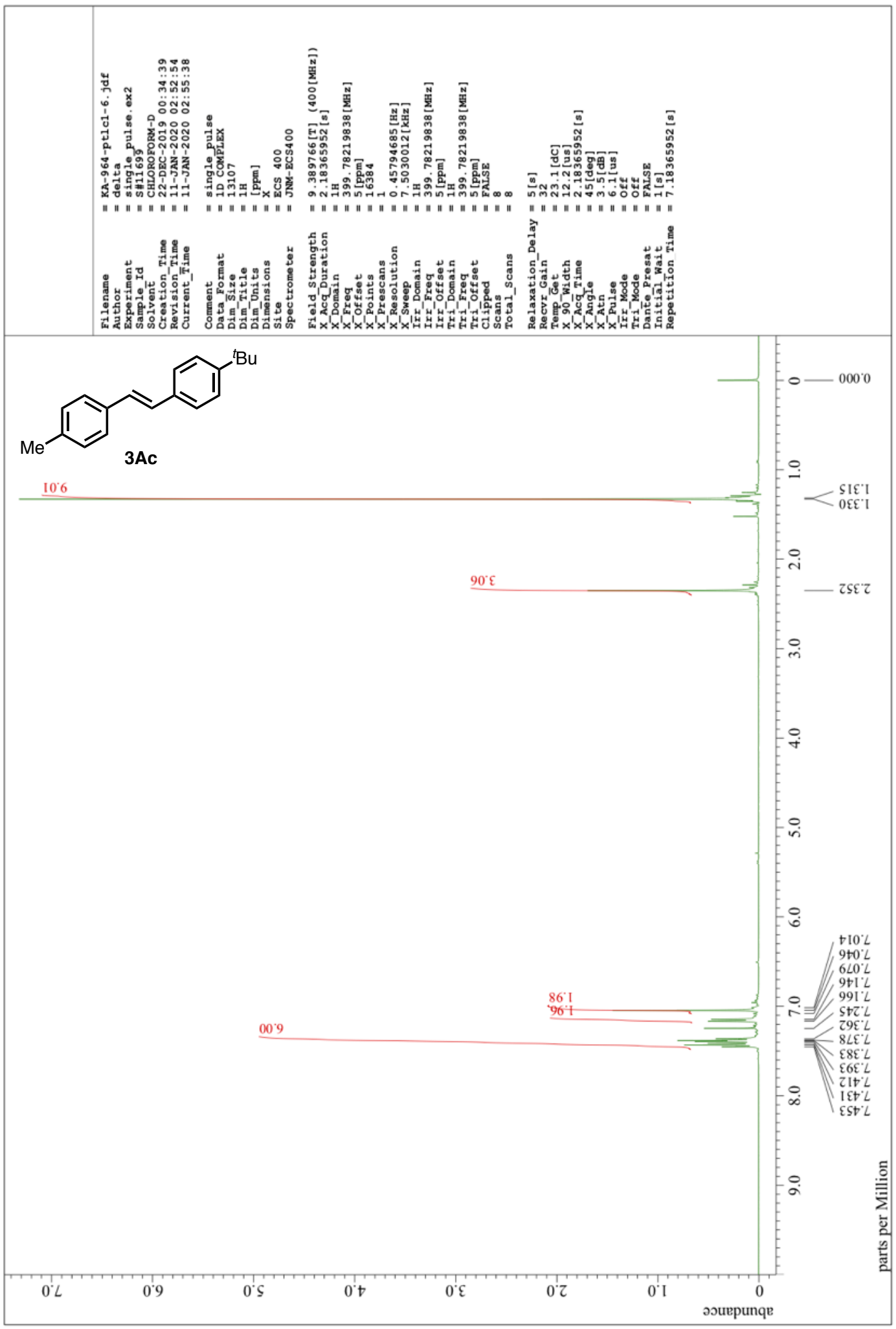


${ }^{13} \mathrm{C}$ NMR of $\mathbf{3 A c}\left(101 \mathrm{MHz}, \mathrm{CDCl}_{3}\right)$

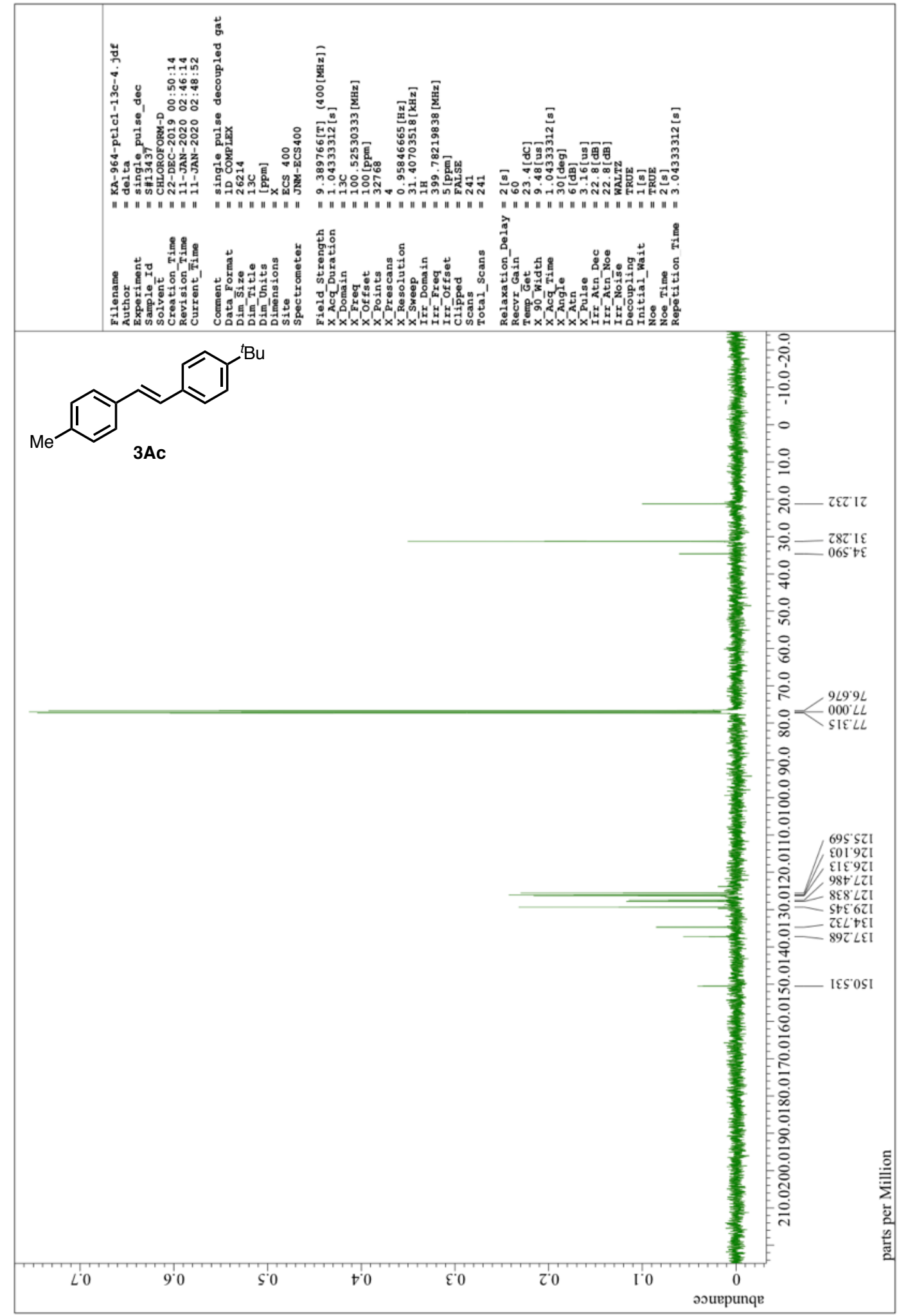


${ }^{1} \mathrm{H}$ NMR of 3Ad $\left(400 \mathrm{MHz}, \mathrm{CDCl}_{3}\right)$

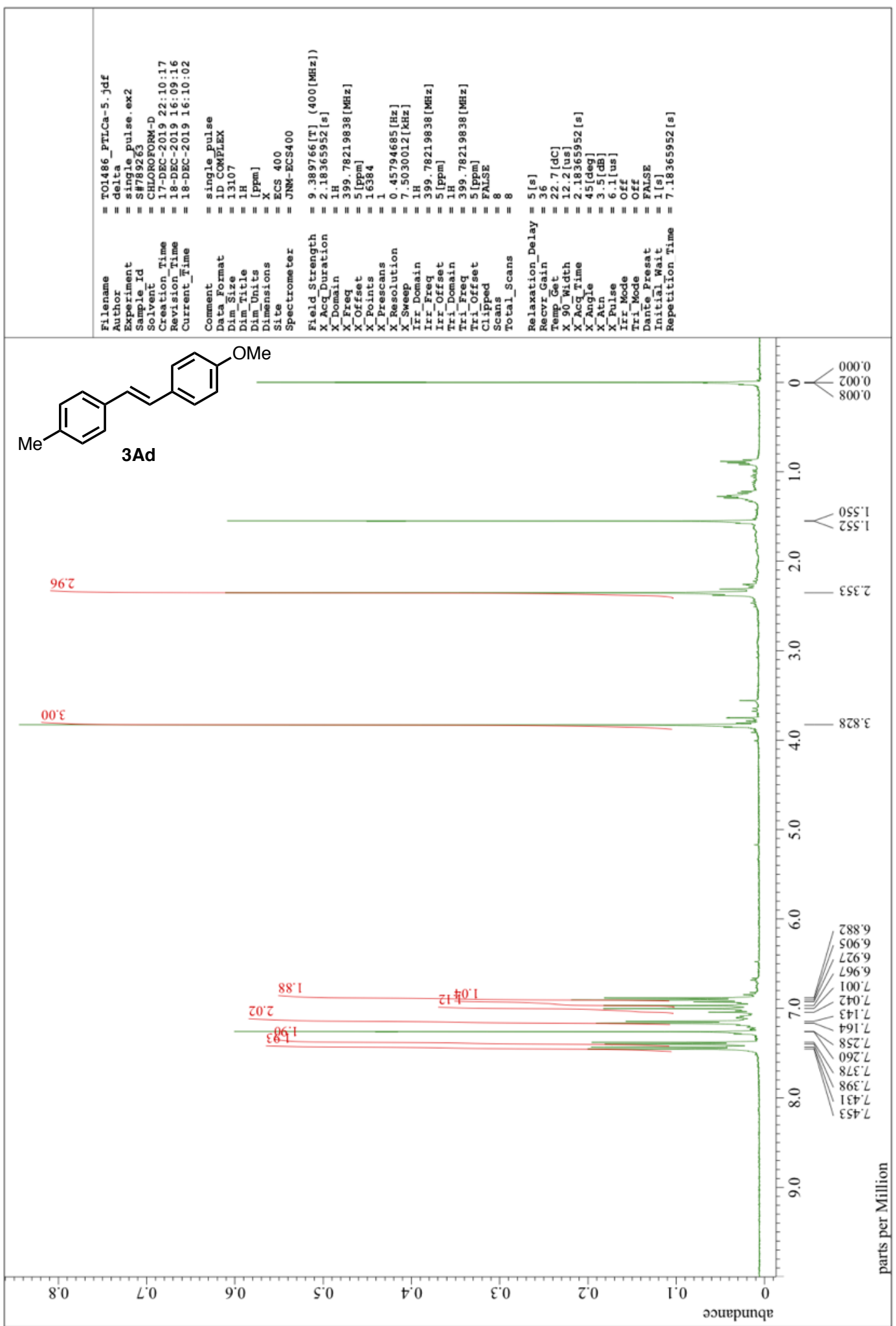


${ }^{13} \mathrm{C}$ NMR of $3 \mathrm{Ad}\left(101 \mathrm{MHz}, \mathrm{CDCl}_{3}\right)$

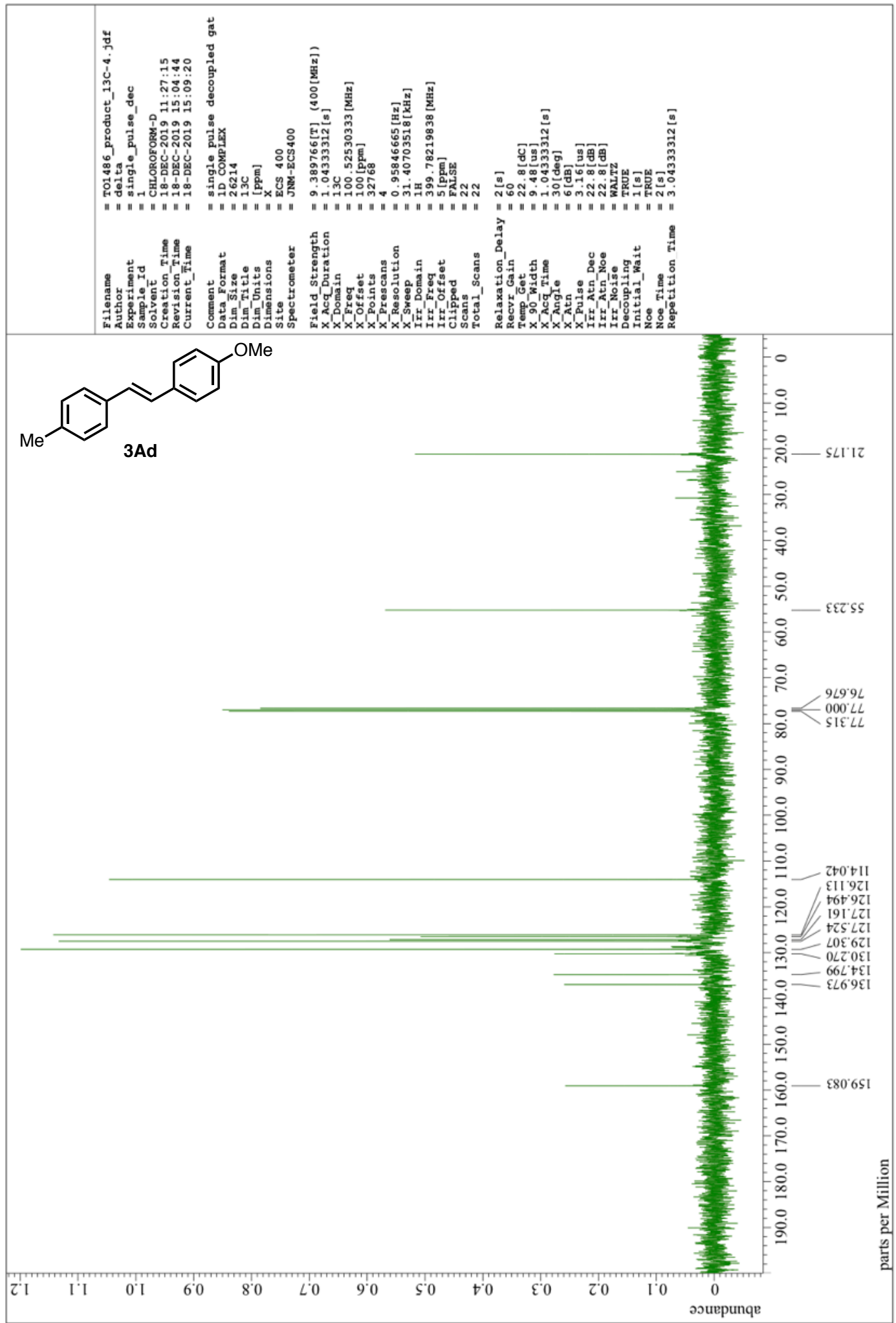


${ }^{1} \mathrm{H}$ NMR of $\mathbf{3 A e}\left(400 \mathrm{MHz}, \mathrm{CDCl}_{3}\right)$

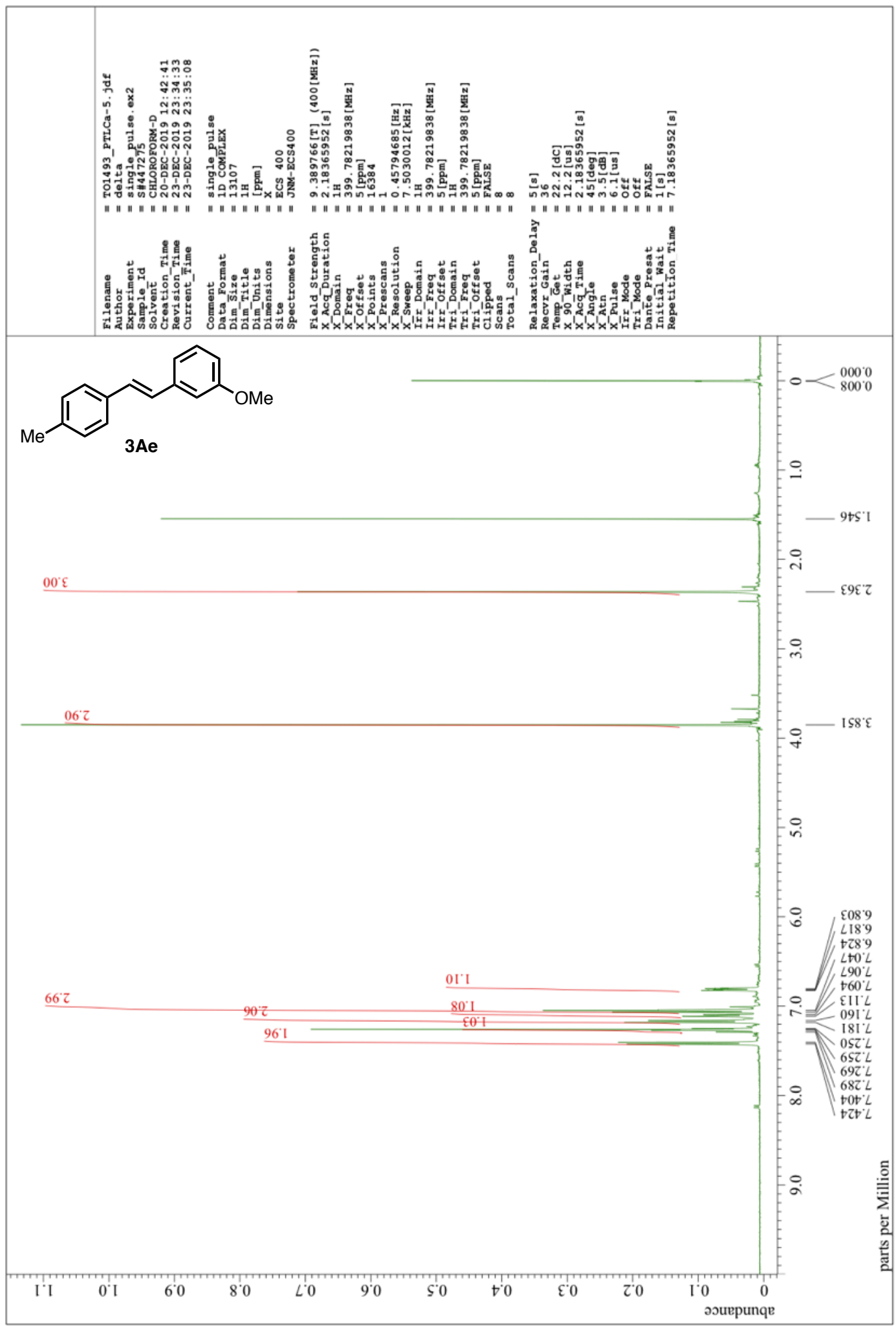


${ }^{13} \mathrm{C}$ NMR of $\mathbf{3 A e}\left(101 \mathrm{MHz}, \mathrm{CDCl}_{3}\right)$

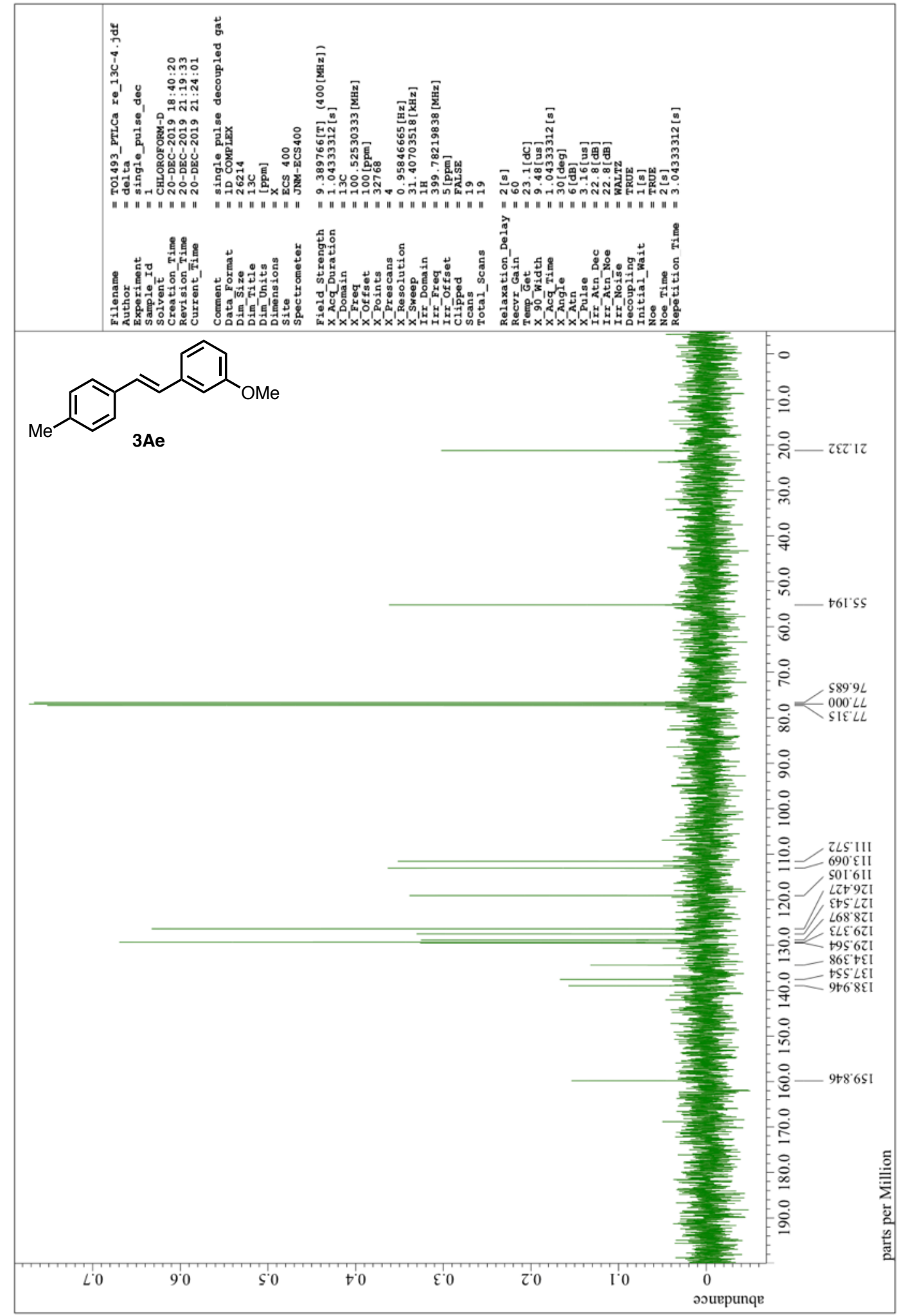


${ }^{1} \mathrm{H}$ NMR of $\mathbf{3 A f}\left(400 \mathrm{MHz}, \mathrm{CDCl}_{3}\right)$

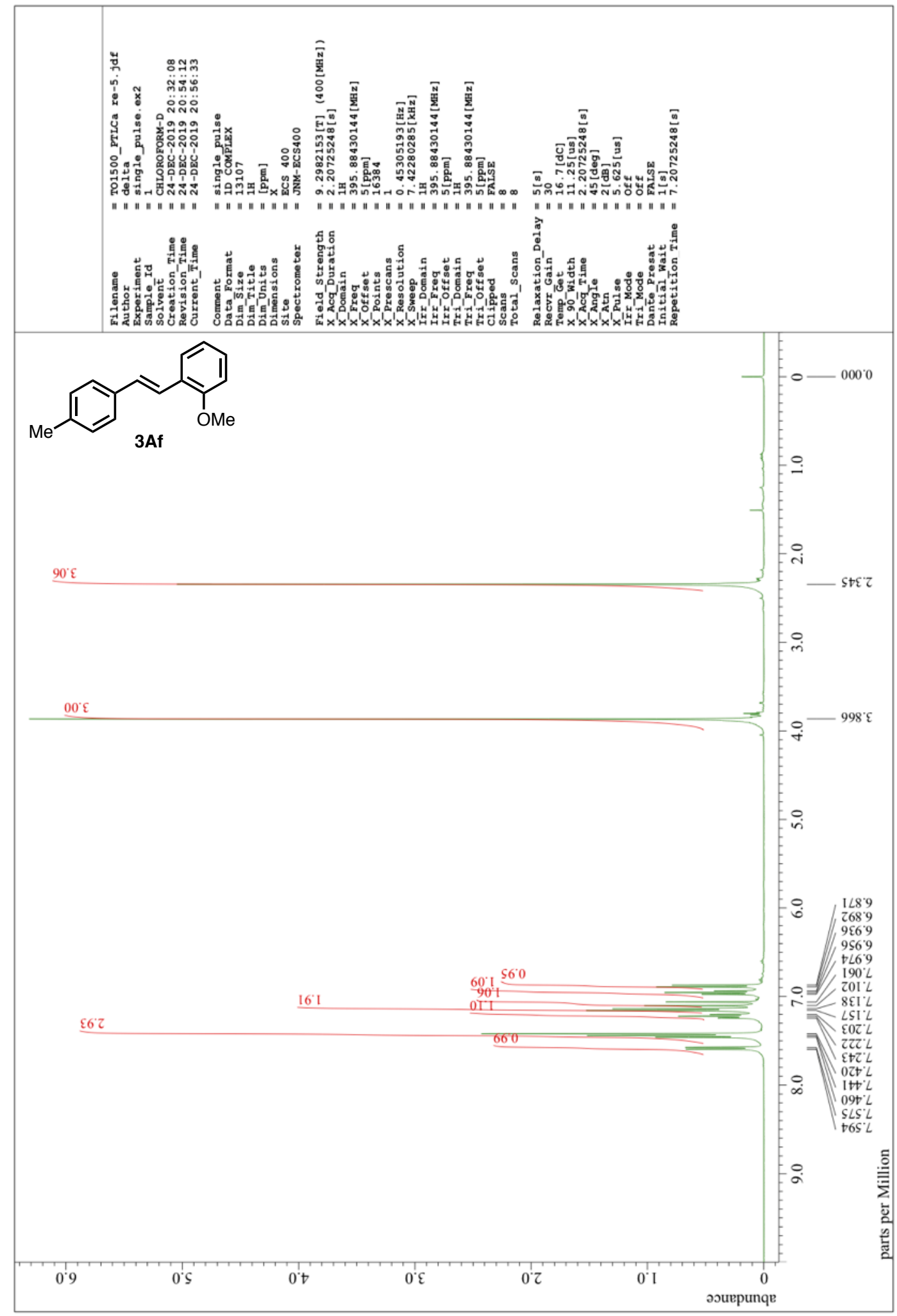


${ }^{13} \mathrm{C}$ NMR of $3 \mathbf{A f}\left(101 \mathrm{MHz}, \mathrm{CDCl}_{3}\right)$

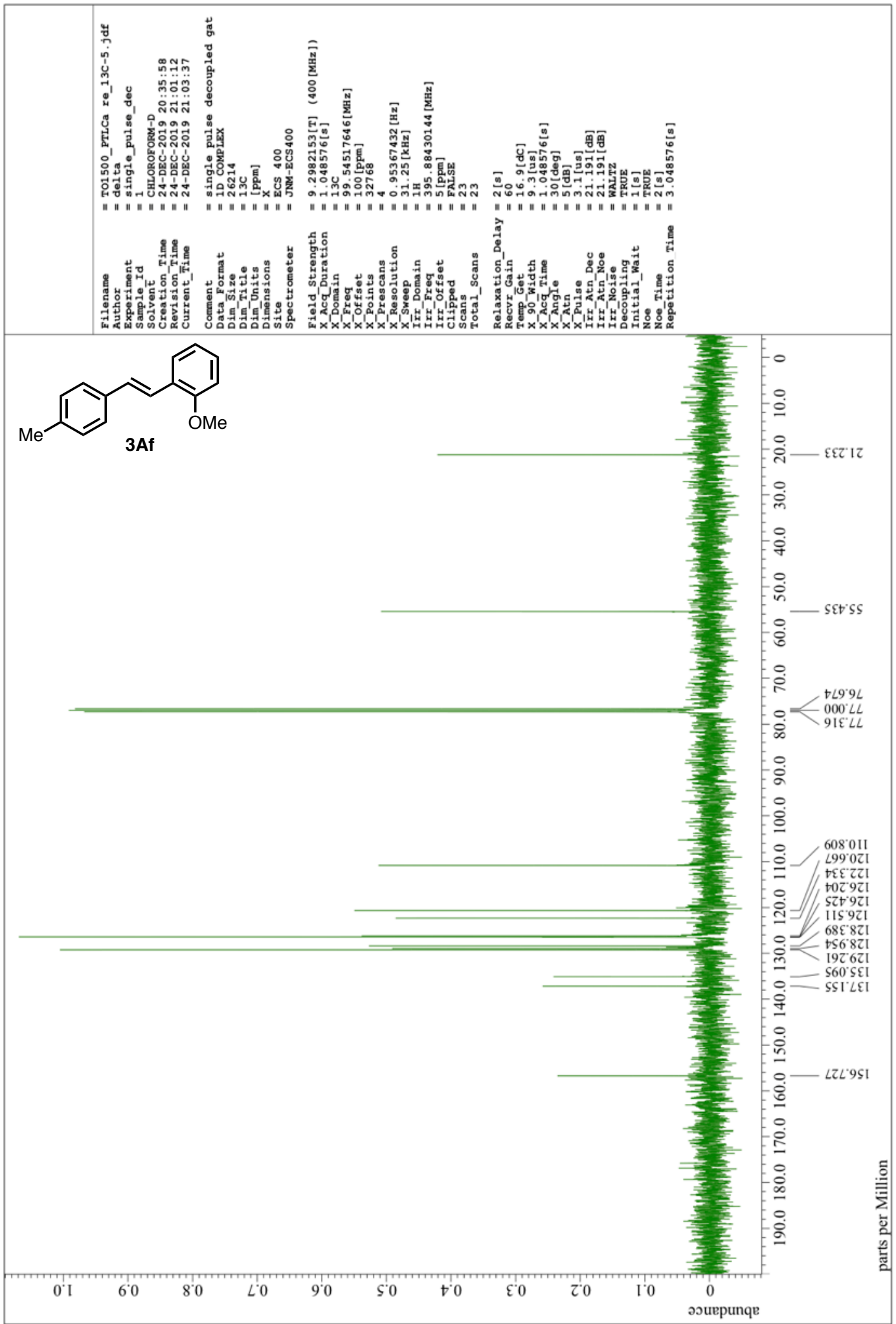


${ }^{1} \mathrm{H}$ NMR of $\mathbf{3 A g}\left(400 \mathrm{MHz}, \mathrm{CDCl}_{3}\right)$

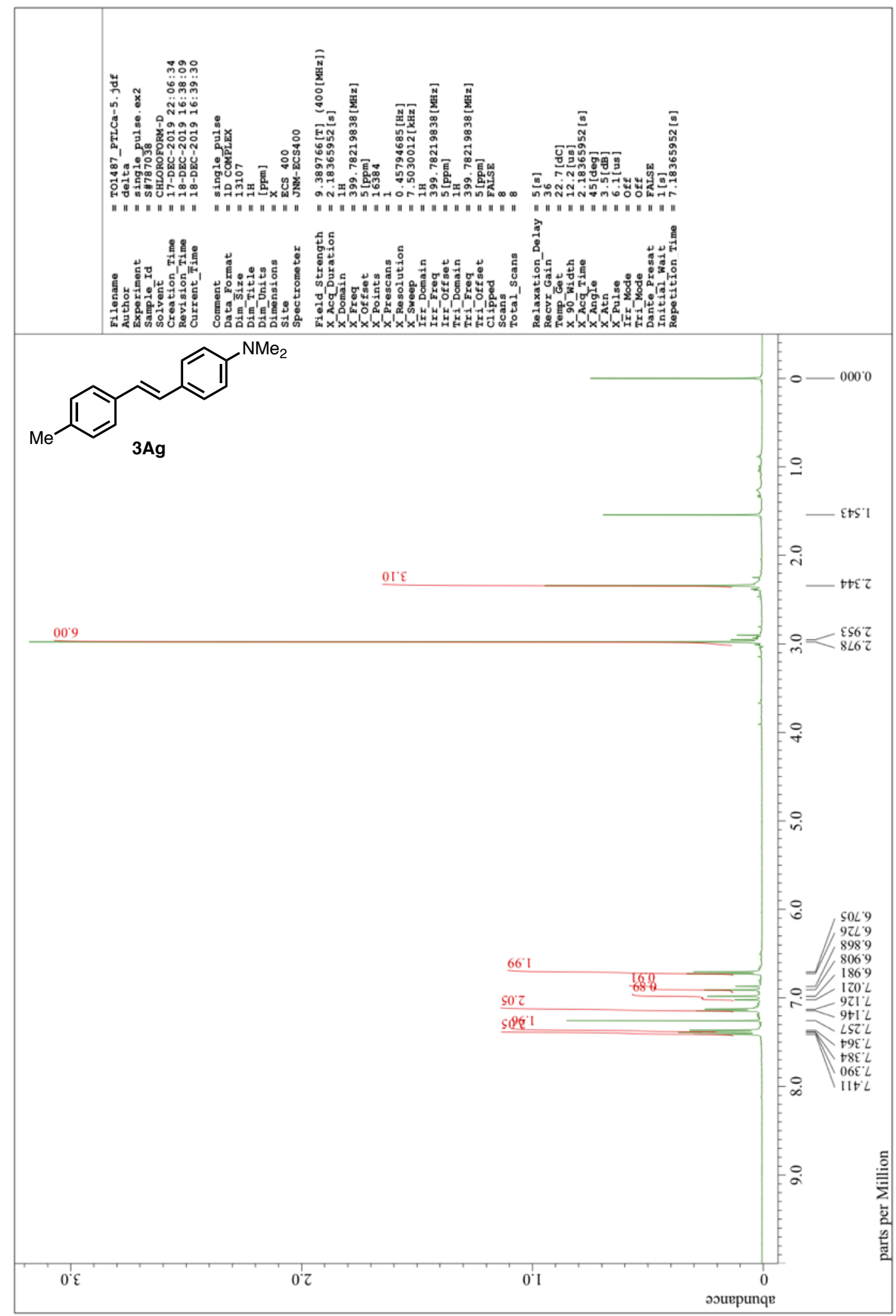


${ }^{13} \mathrm{C}$ NMR of $\mathbf{3 A g}\left(101 \mathrm{MHz}, \mathrm{CDCl}_{3}\right)$

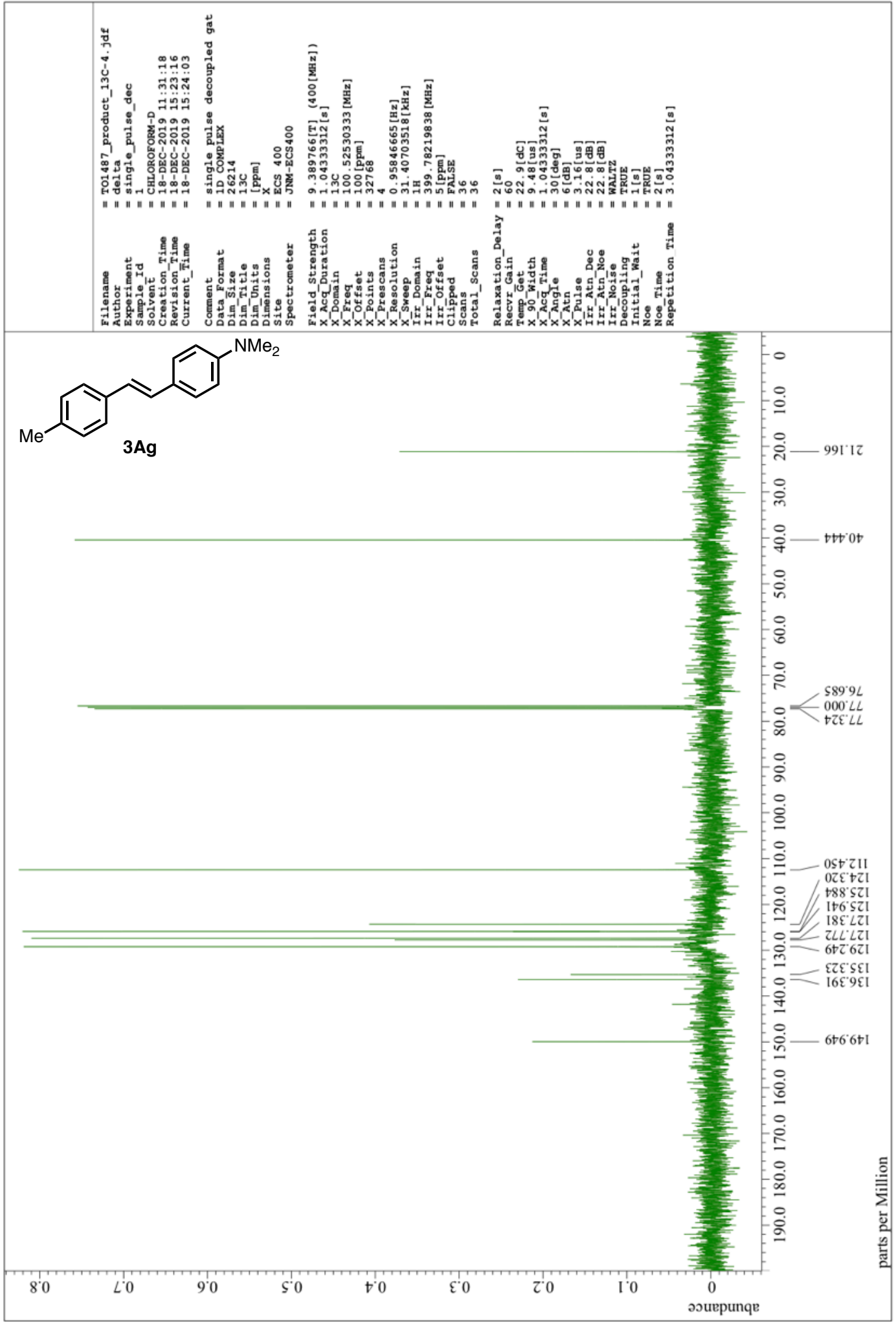


${ }^{1} \mathrm{H}$ NMR of $\mathbf{3 A h}\left(400 \mathrm{MHz}, \mathrm{CDCl}_{3}\right)$

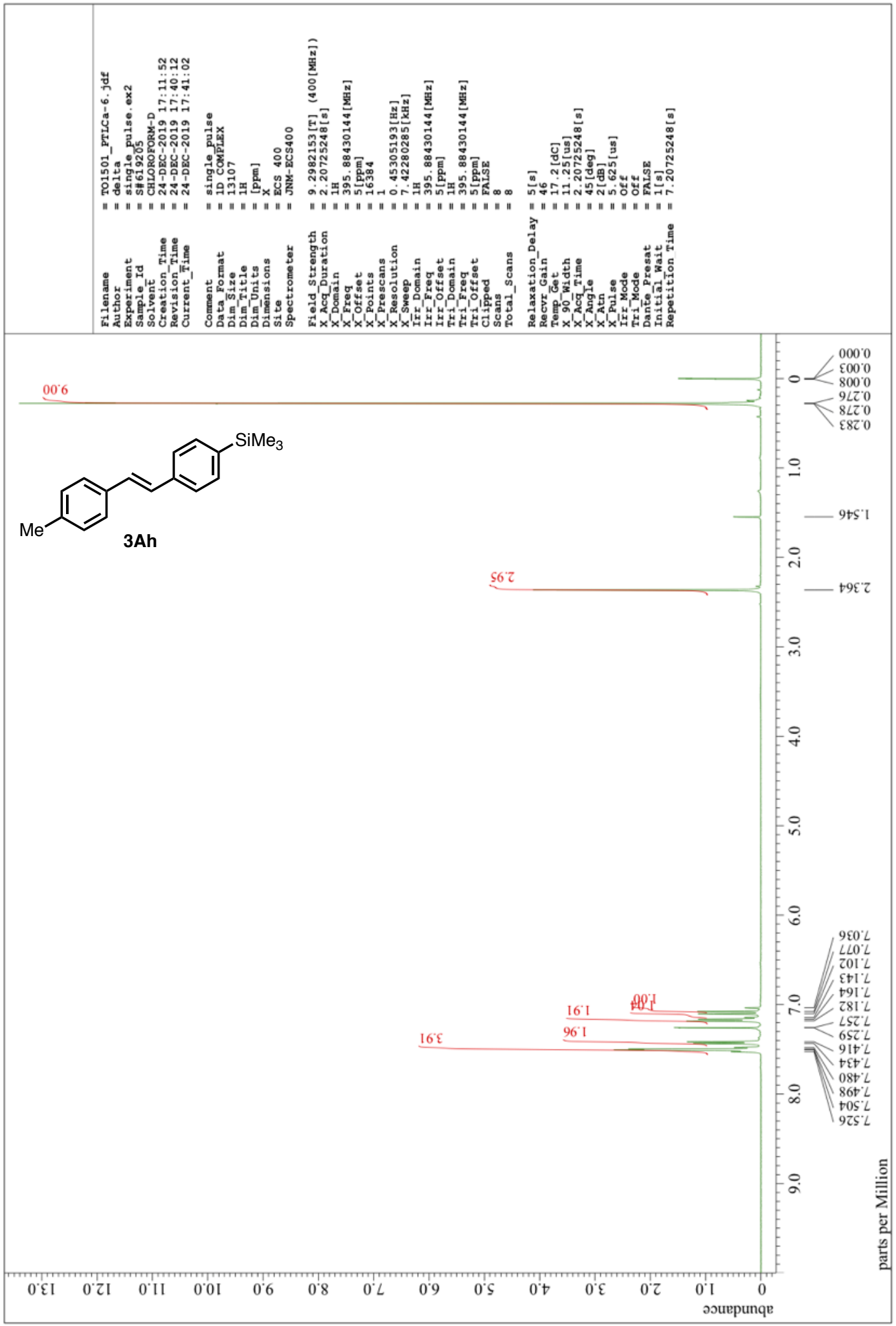


${ }^{13} \mathrm{C}$ NMR of $\mathbf{3 A h}\left(101 \mathrm{MHz}, \mathrm{CDCl}_{3}\right)$

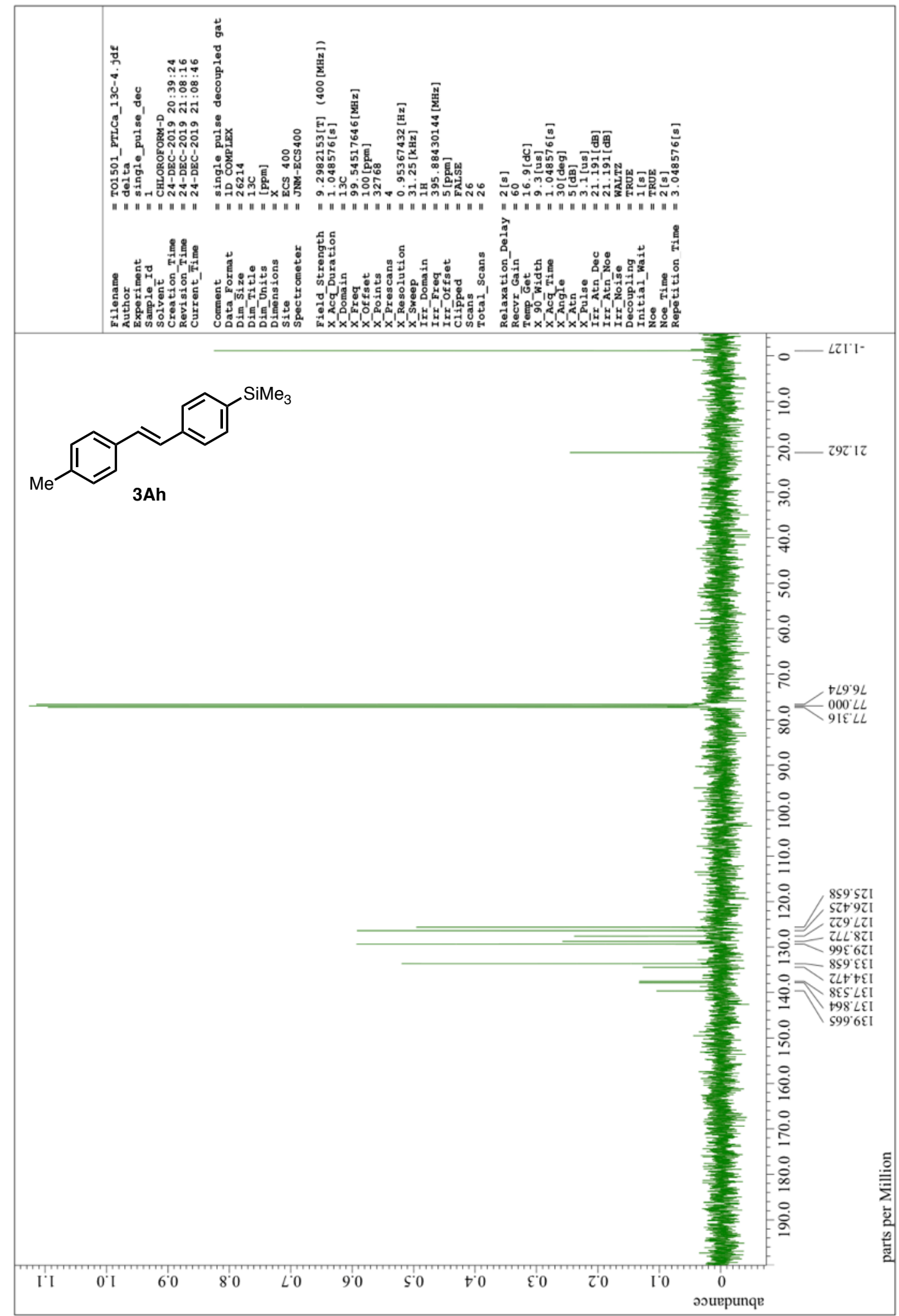


${ }^{1} \mathrm{H}$ NMR of $\mathbf{3 A i}\left(400 \mathrm{MHz}, \mathrm{CDCl}_{3}\right)$

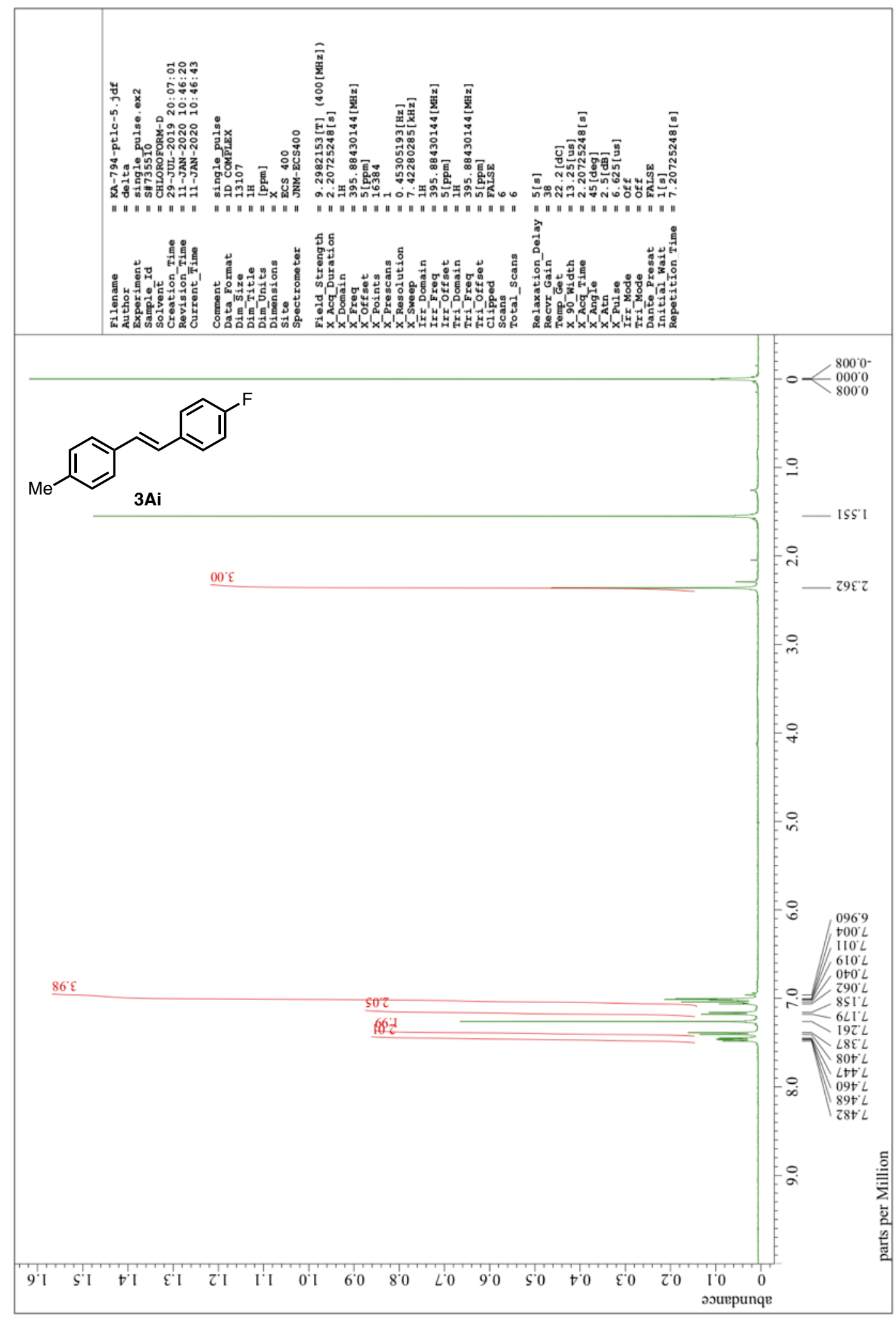


${ }^{13} \mathrm{C}$ NMR of 3Ai $\left(101 \mathrm{MHz}, \mathrm{CDCl}_{3}\right)$

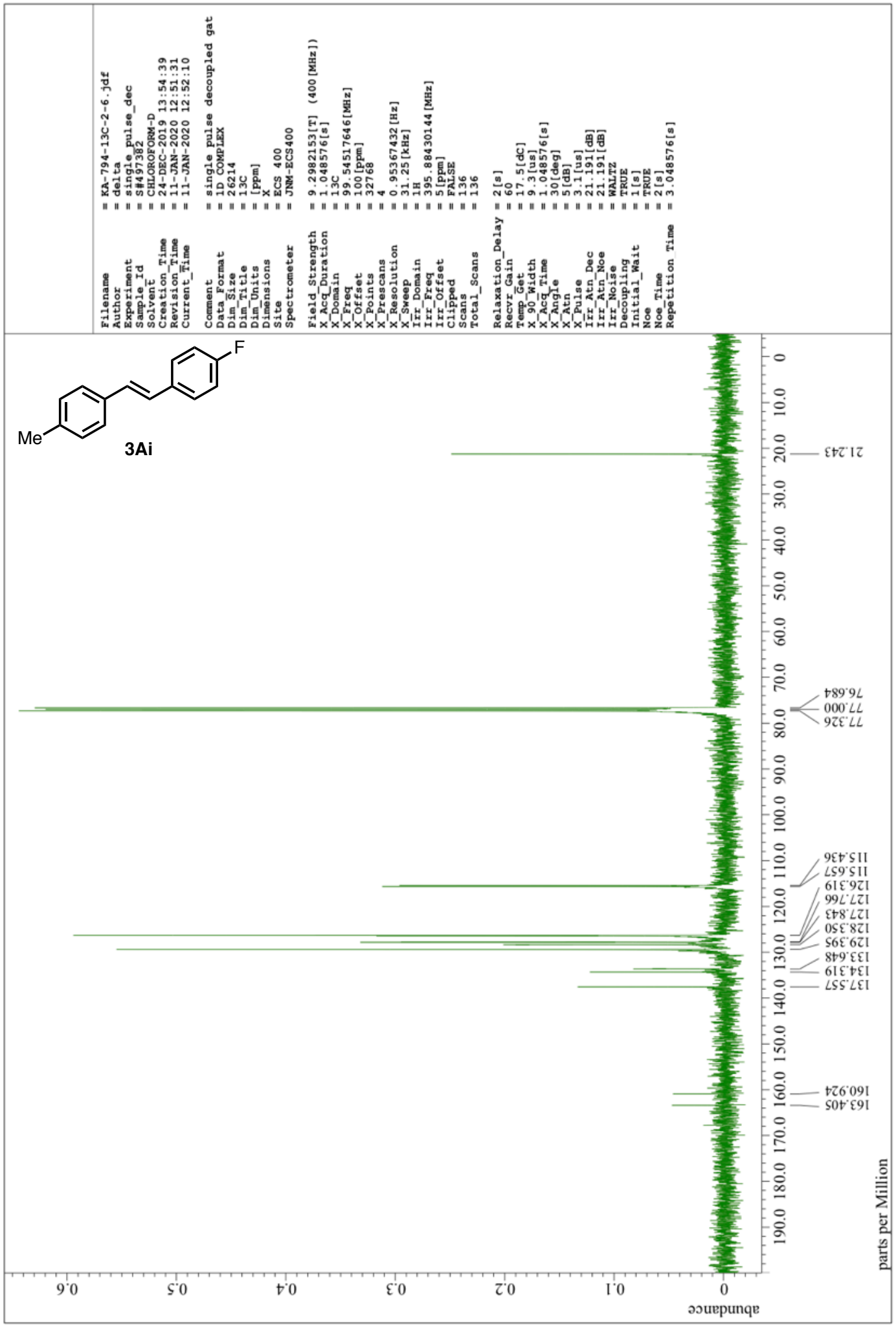


${ }^{1} \mathrm{H}$ NMR of $\mathbf{3 A j}\left(400 \mathrm{MHz}, \mathrm{CDCl}_{3}\right)$

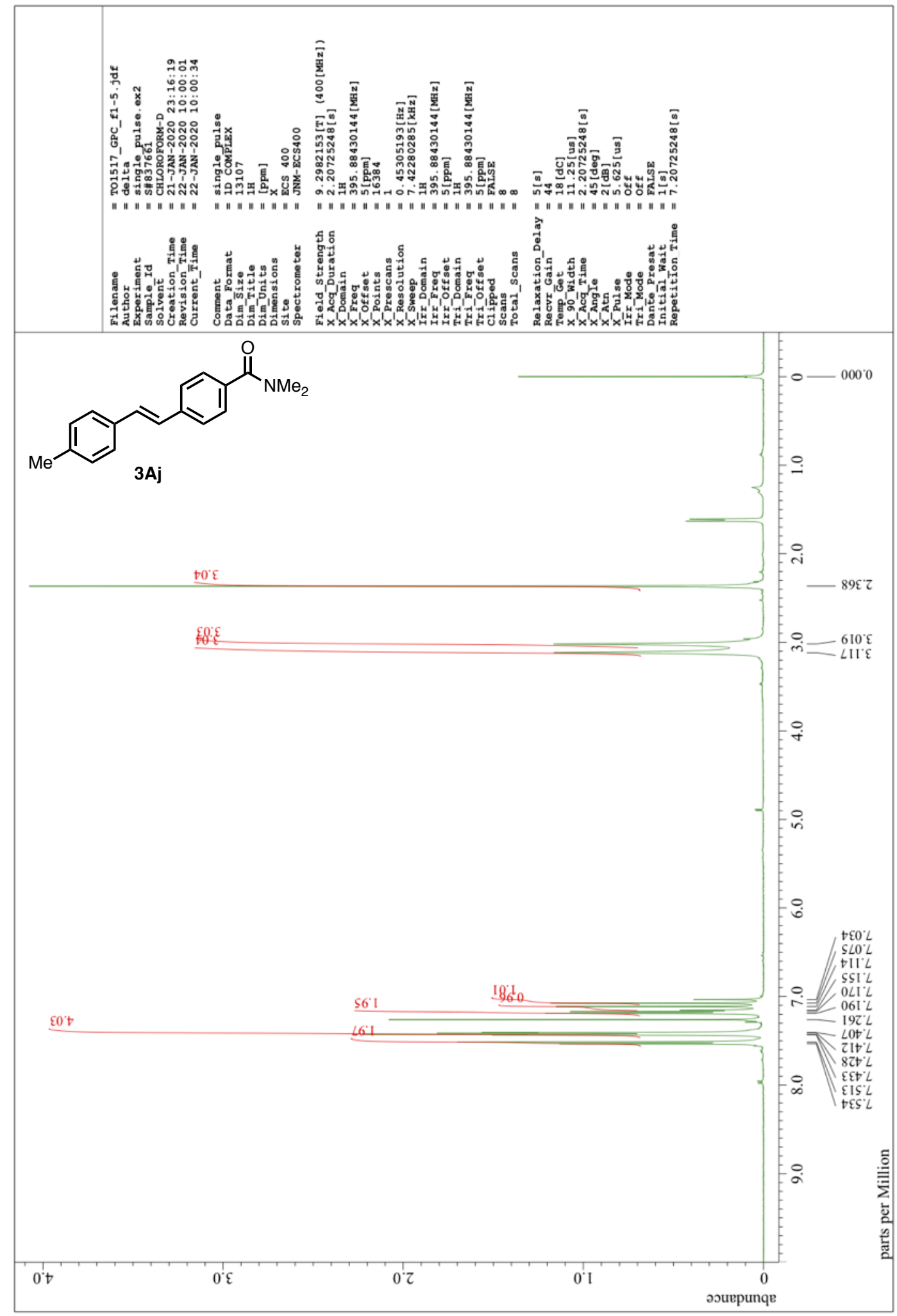


${ }^{13} \mathrm{C}$ NMR of $\mathbf{3 A j}\left(101 \mathrm{MHz}, \mathrm{CDCl}_{3}\right)$

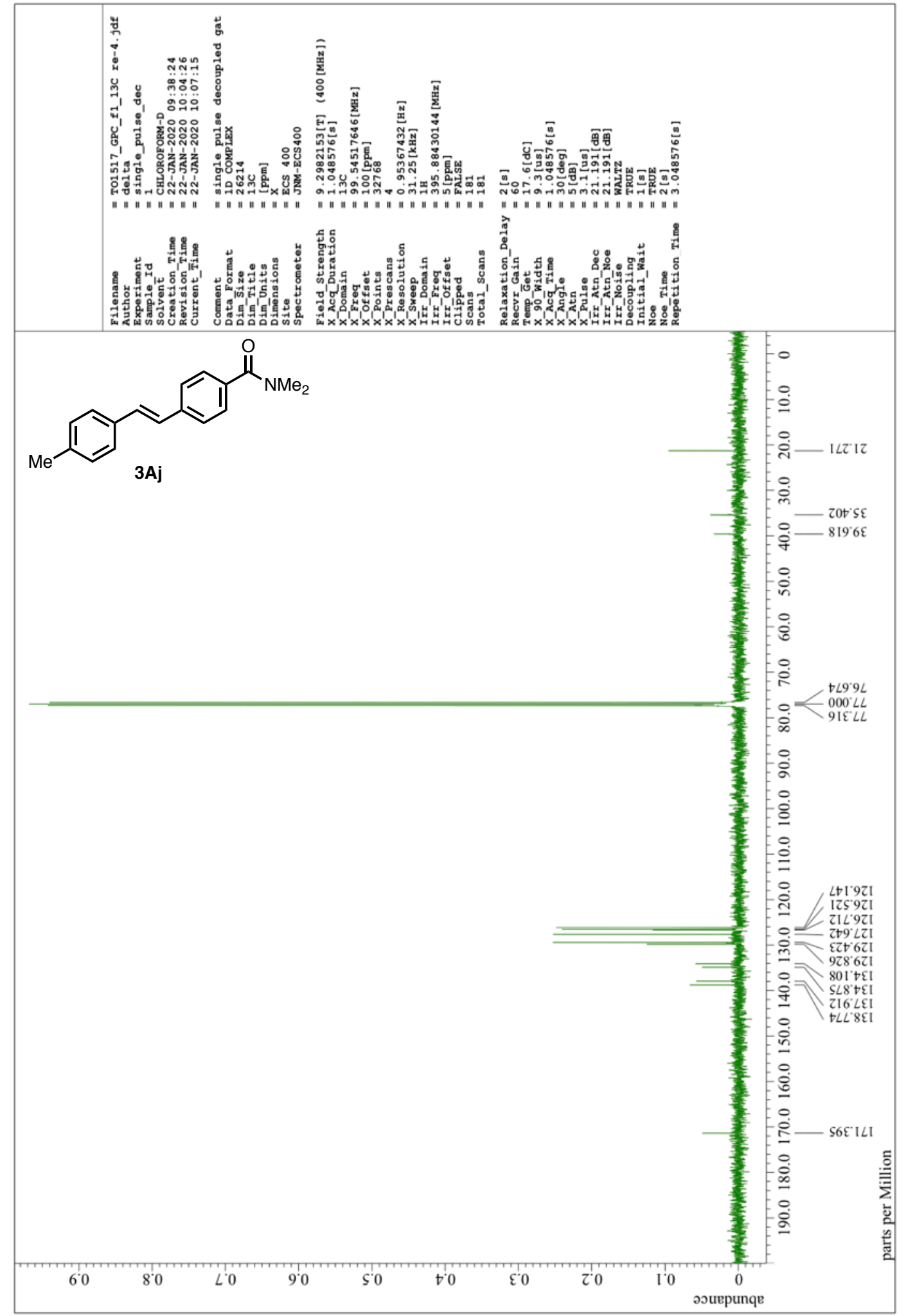


${ }^{1} \mathrm{H}$ NMR of $\mathbf{3 A k}\left(400 \mathrm{MHz}, \mathrm{CDCl}_{3}\right)$

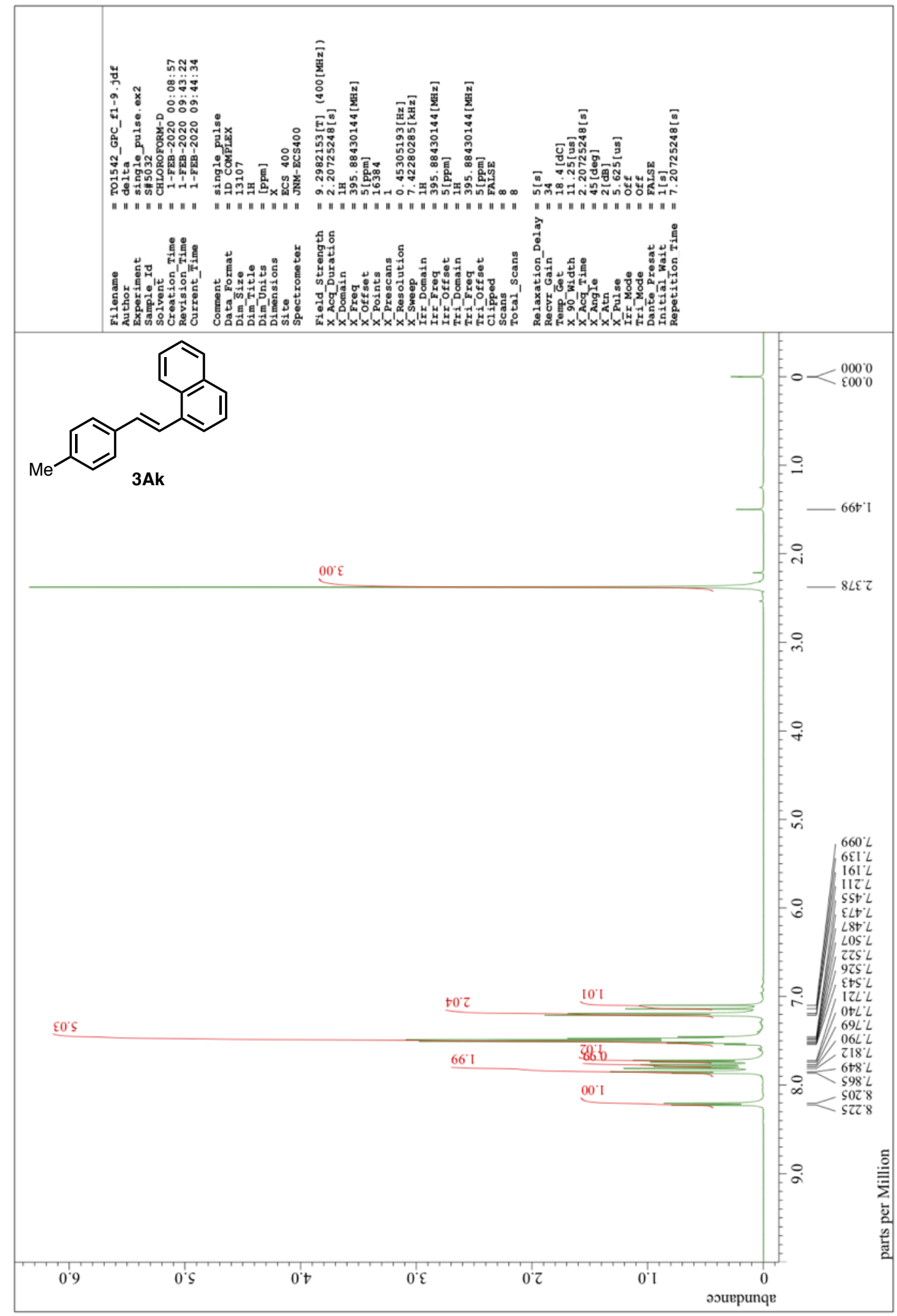


${ }^{13} \mathrm{C}$ NMR of $\mathbf{3 A k}\left(101 \mathrm{MHz}, \mathrm{CDCl}_{3}\right)$

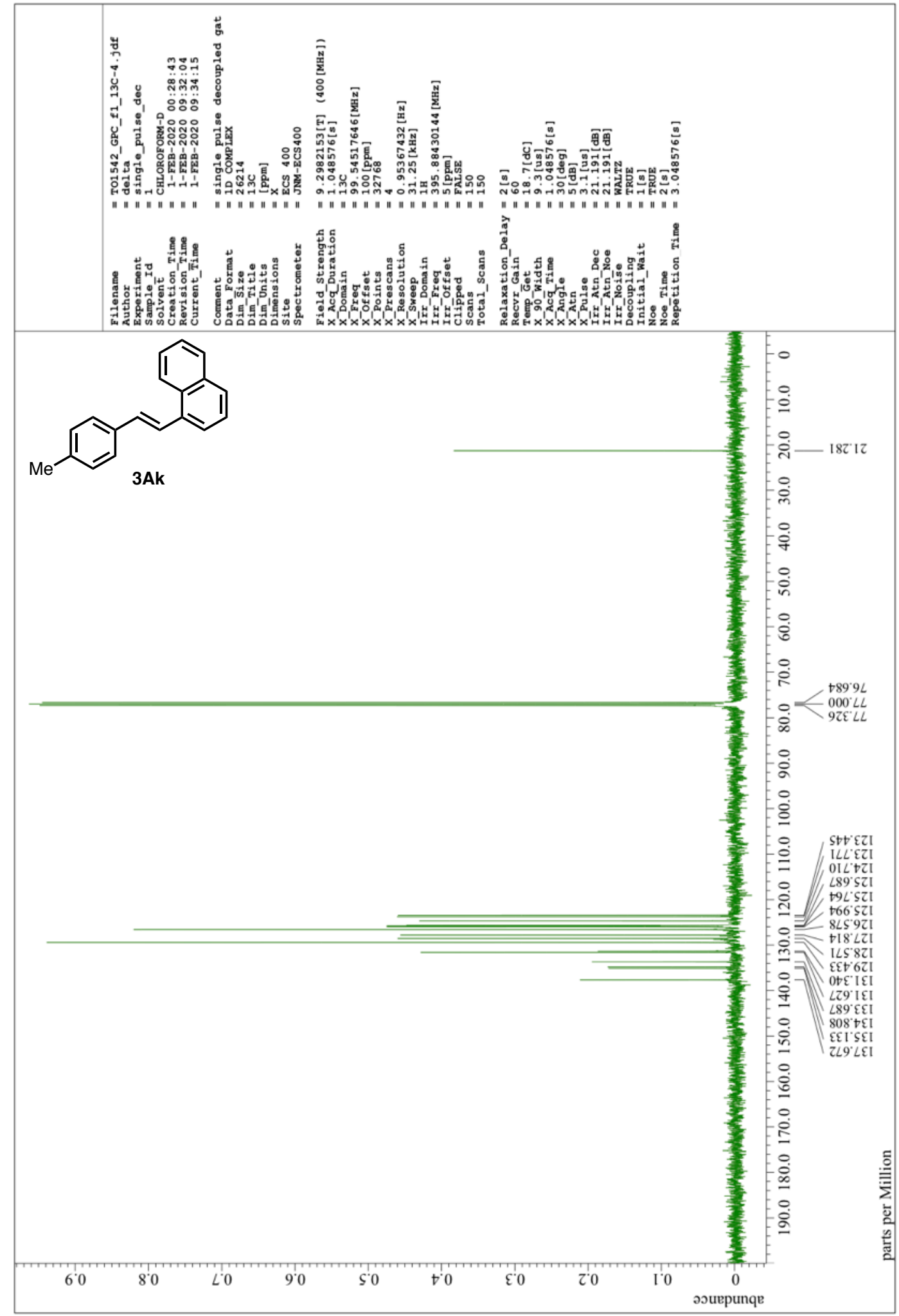


${ }^{1} \mathrm{H}$ NMR of $\mathbf{3 A l}\left(400 \mathrm{MHz}, \mathrm{CDCl}_{3}\right)$

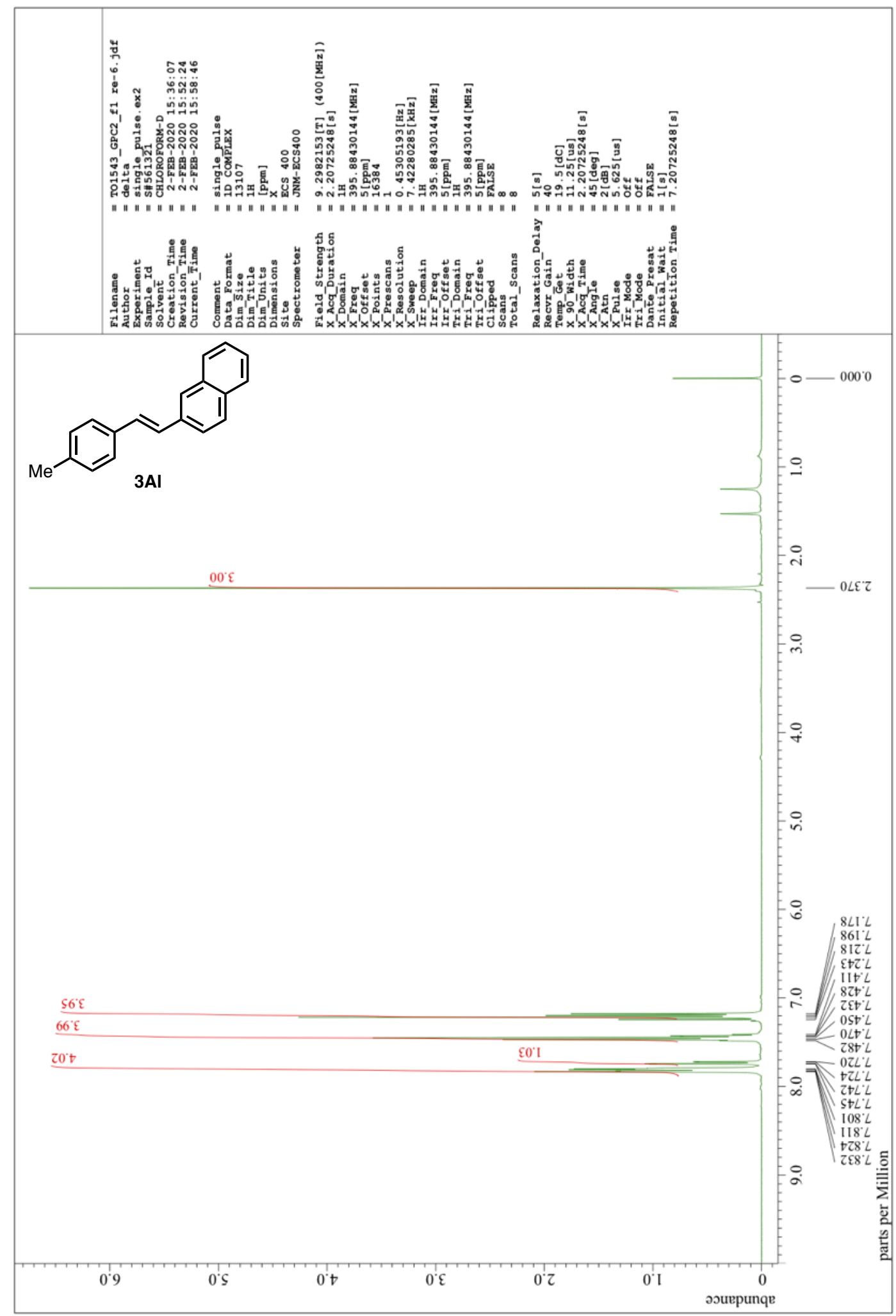


${ }^{13} \mathrm{C}$ NMR of 3Al $\left(101 \mathrm{MHz}, \mathrm{CDCl}_{3}\right)$

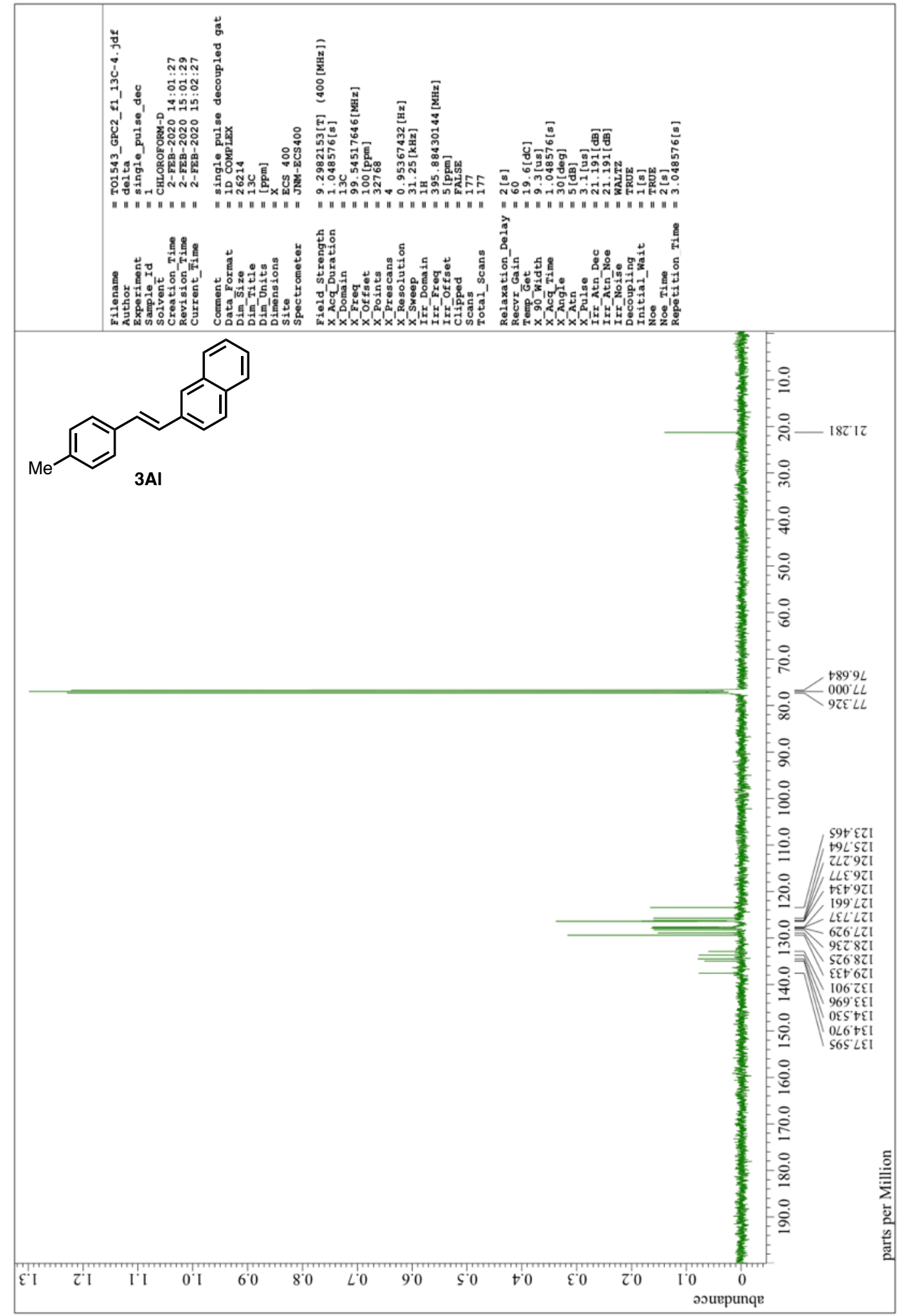


${ }^{1} \mathrm{H}$ NMR of $\mathbf{3 A m}\left(400 \mathrm{MHz}, \mathrm{CDCl}_{3}\right)$

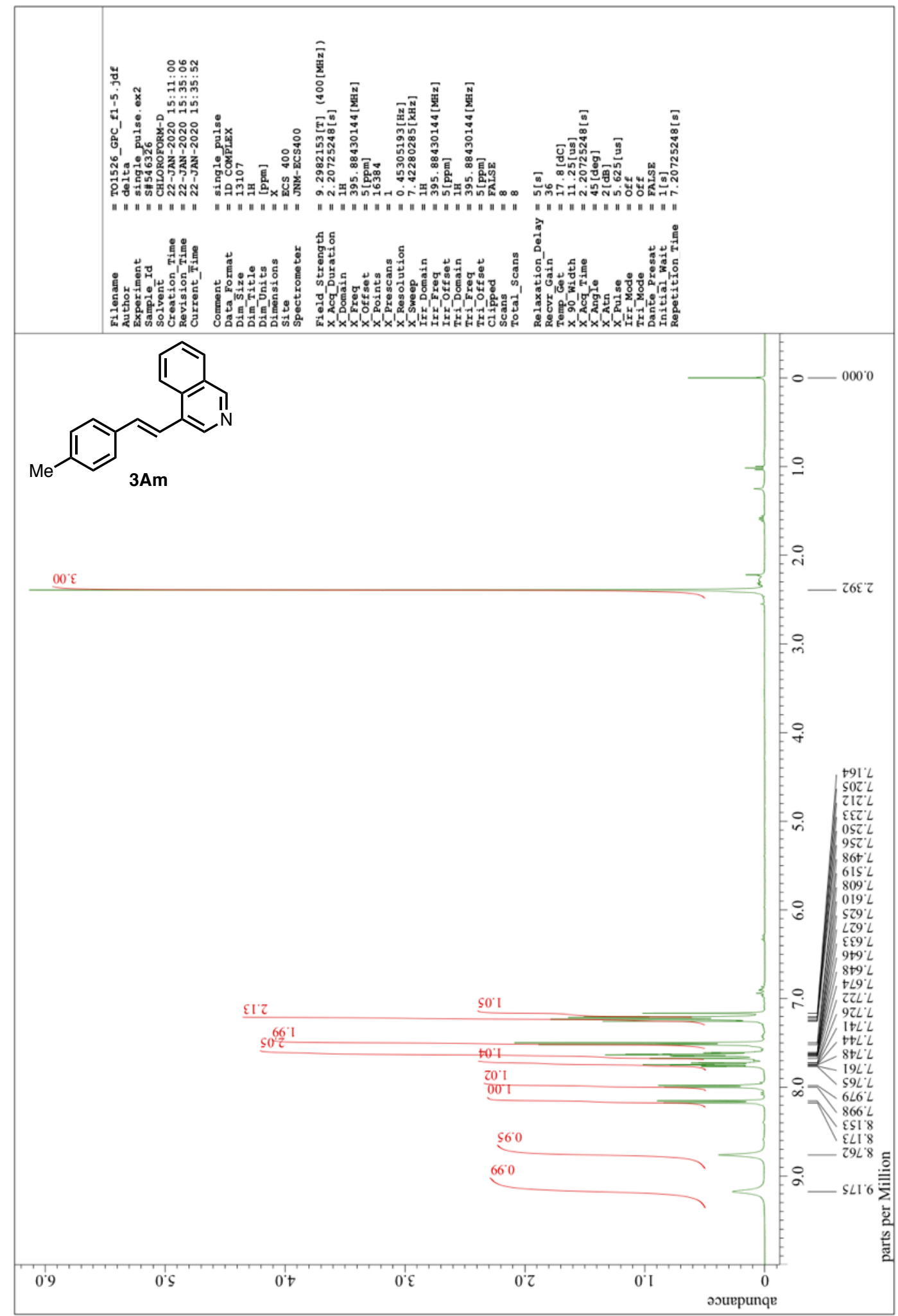


${ }^{13} \mathrm{C}$ NMR of $\mathbf{3 A m}\left(101 \mathrm{MHz}, \mathrm{CDCl}_{3}\right)$

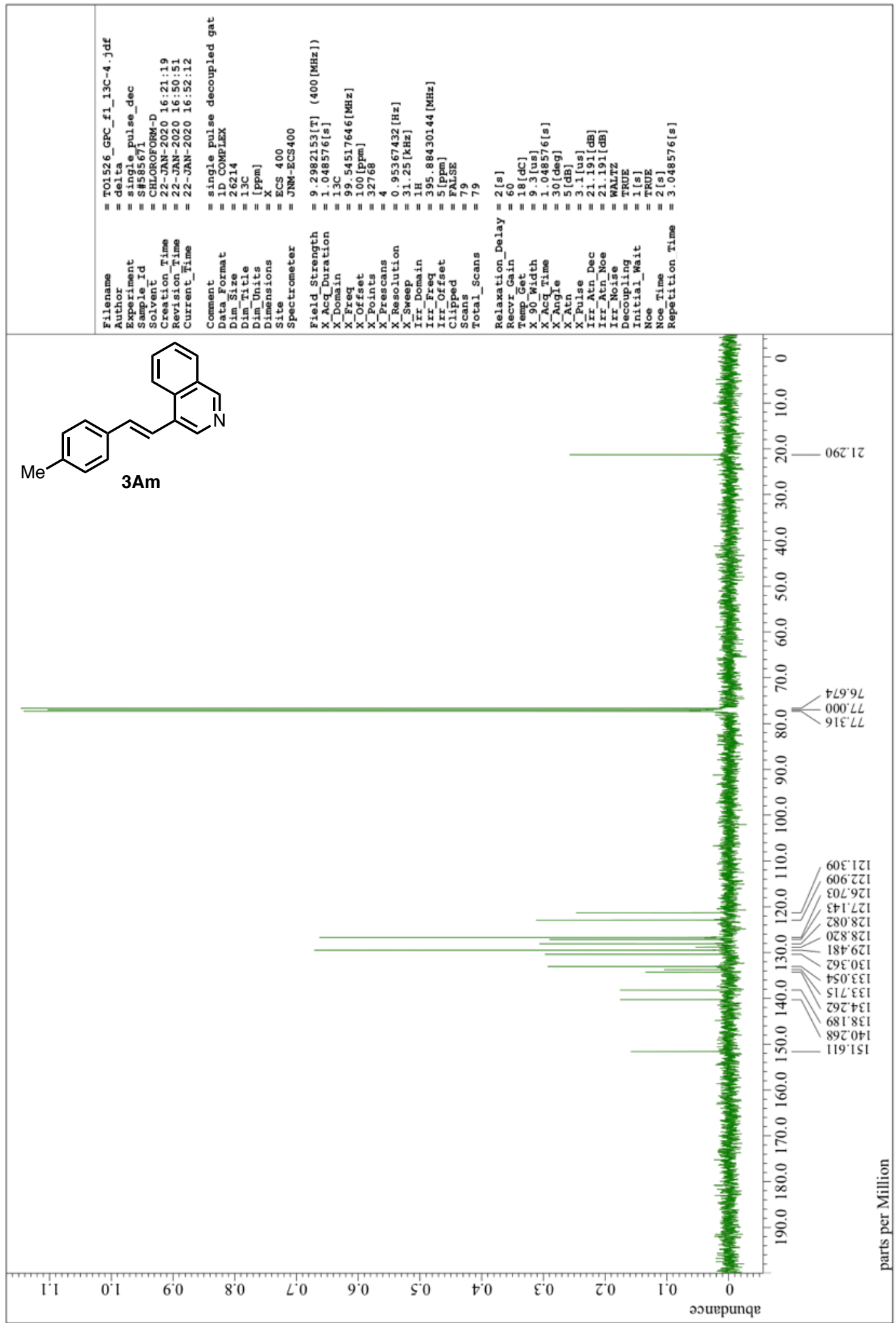


${ }^{1} \mathrm{H}$ NMR of a mixture of $\mathbf{3 A n}, \mathbf{3 A n}$ ' and $\mathbf{3 A n}$ " (400 MHz, $\mathrm{CDCl}_{3}$ )

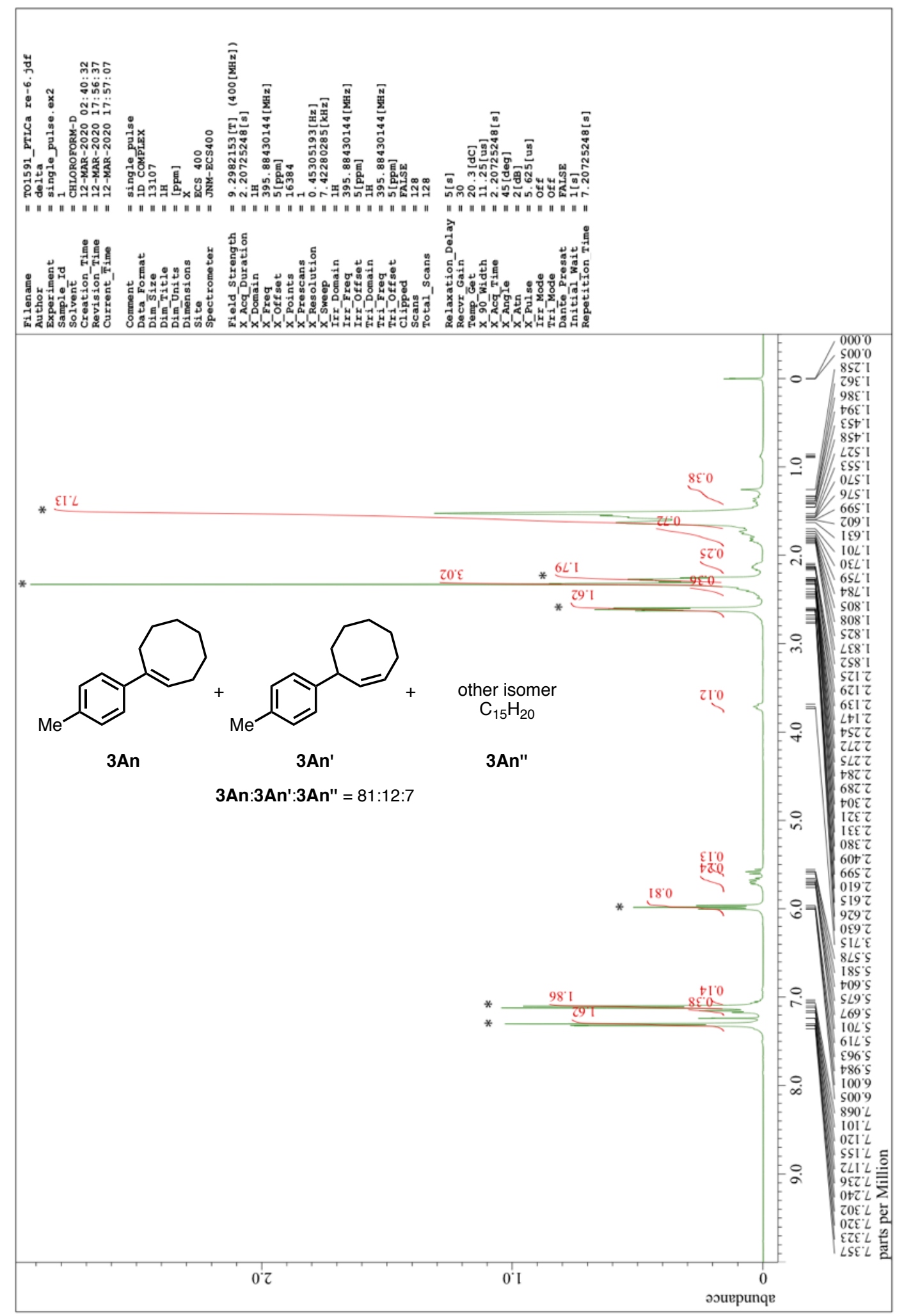


${ }^{13} \mathrm{C}$ NMR of a mixture of $\mathbf{3 A n}, \mathbf{3 A n}$ ' and $\mathbf{3 A n} "\left(101 \mathrm{MHz}, \mathrm{CDCl}_{3}\right)$

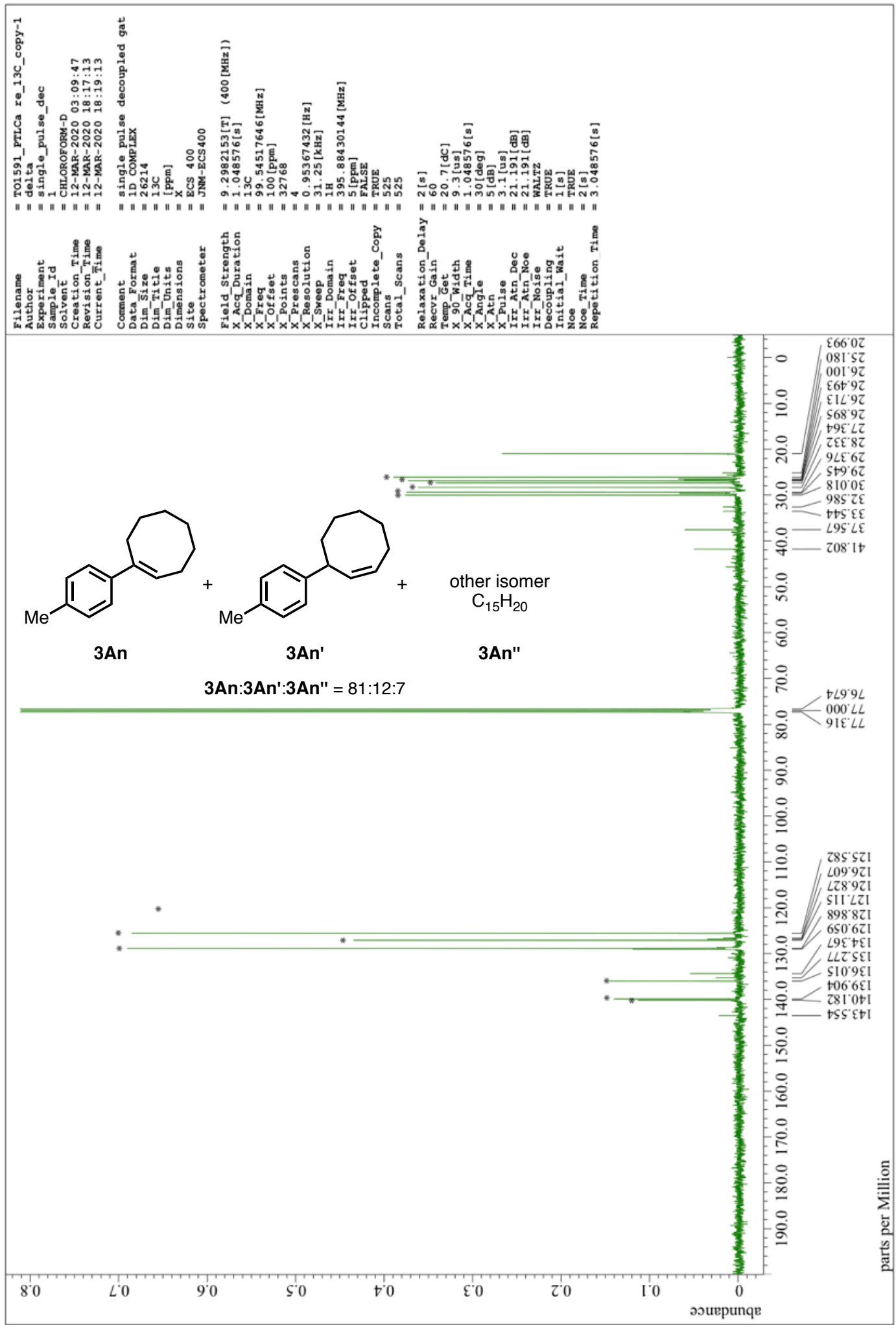


${ }^{1} \mathrm{H}$ NMR of $\mathbf{3 A n}\left(400 \mathrm{MHz}, \mathrm{CDCl}_{3}\right)$

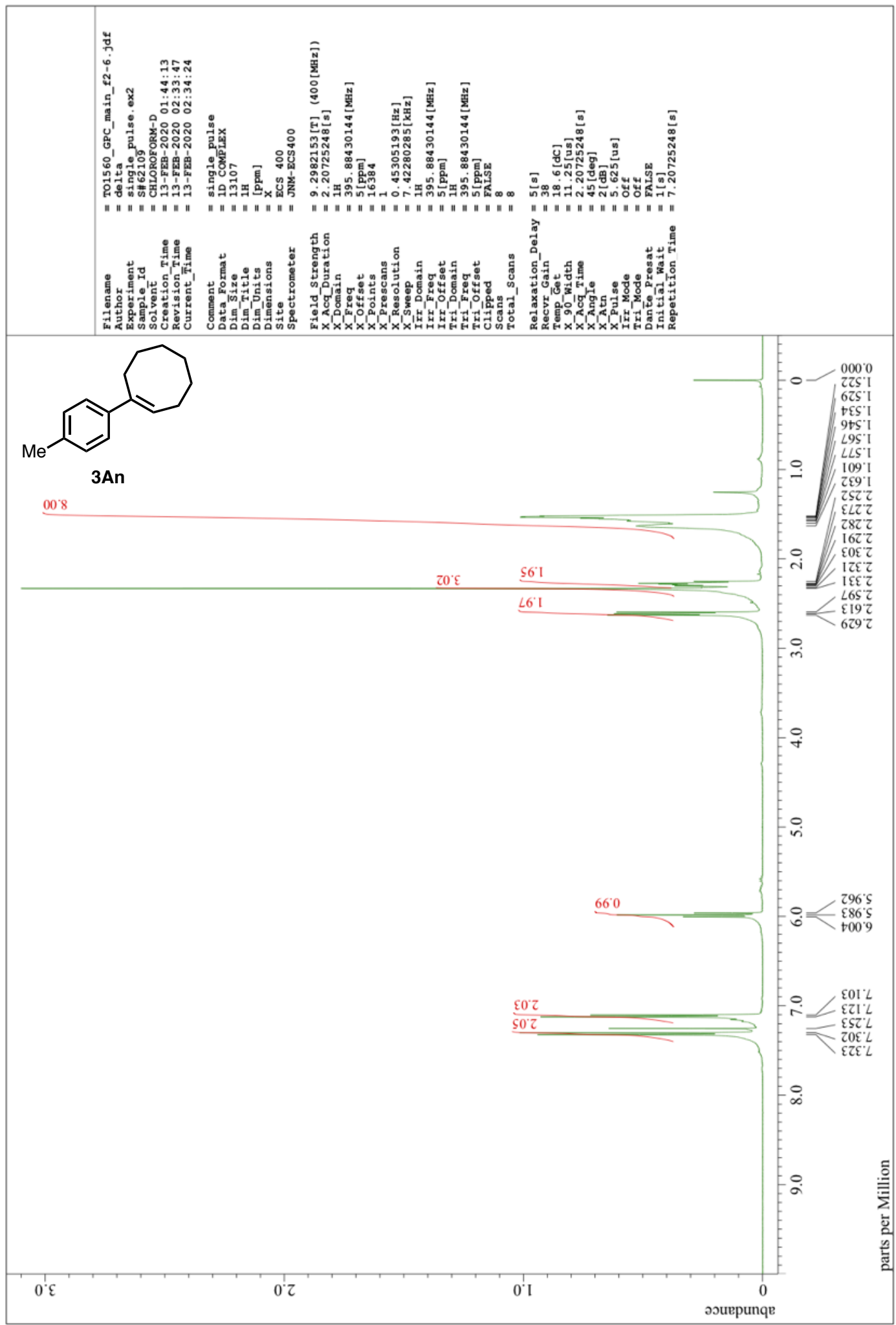


${ }^{13} \mathrm{C}$ NMR of $\mathbf{3 A n}\left(101 \mathrm{MHz}, \mathrm{CDCl}_{3}\right)$

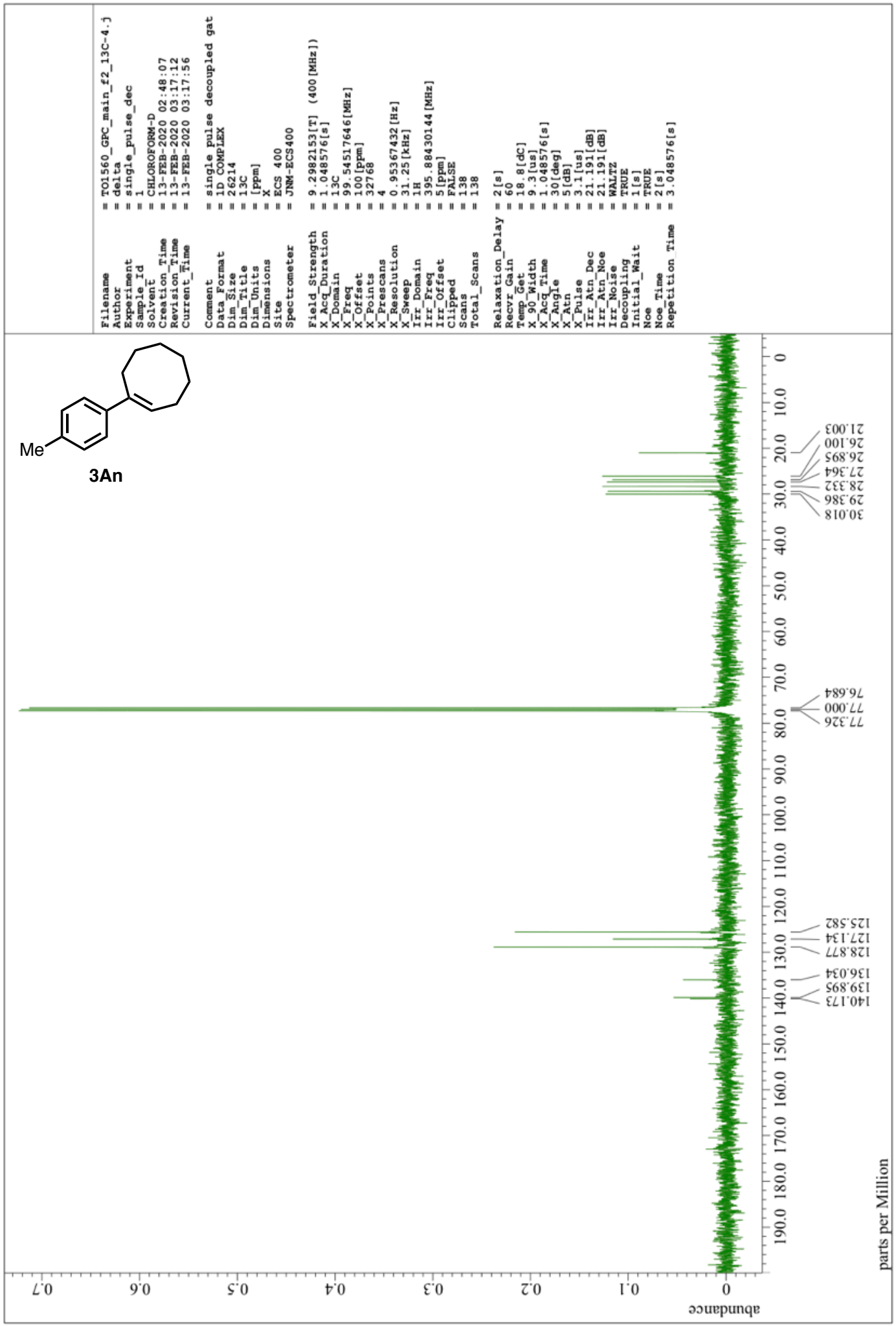


${ }^{1} \mathrm{H}$ NMR of a mixture of (E)-3Ao, (Z)-3Ao, 3Ao' $\left(400 \mathrm{MHz}, \mathrm{CDCl}_{3}\right)$

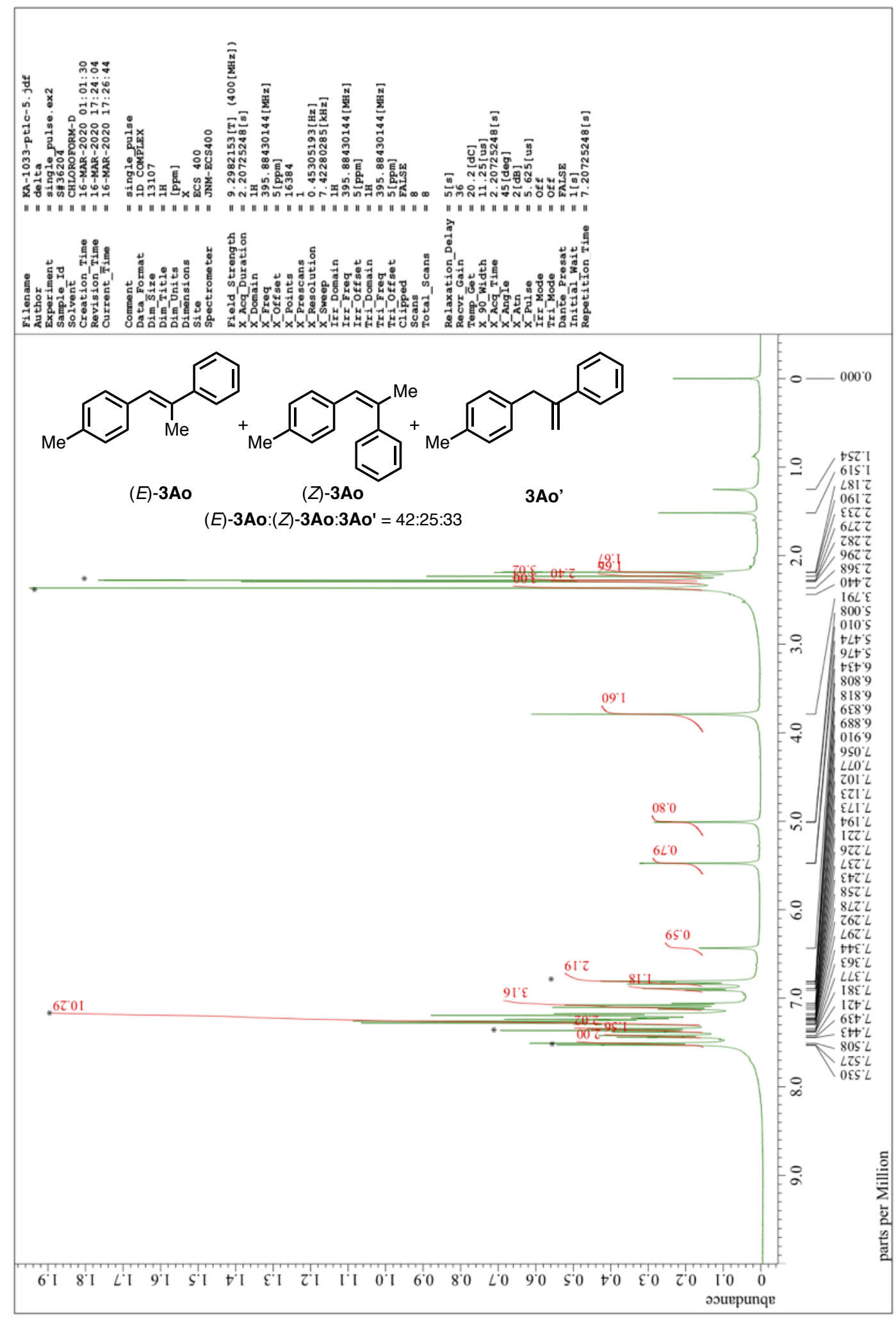


${ }^{13} \mathrm{C}$ NMR of a mixture of (E)-3Ao, $(Z)-\mathbf{3 A o}, \mathbf{3 A o}\left(101 \mathrm{MHz}, \mathrm{CDCl}_{3}\right)$

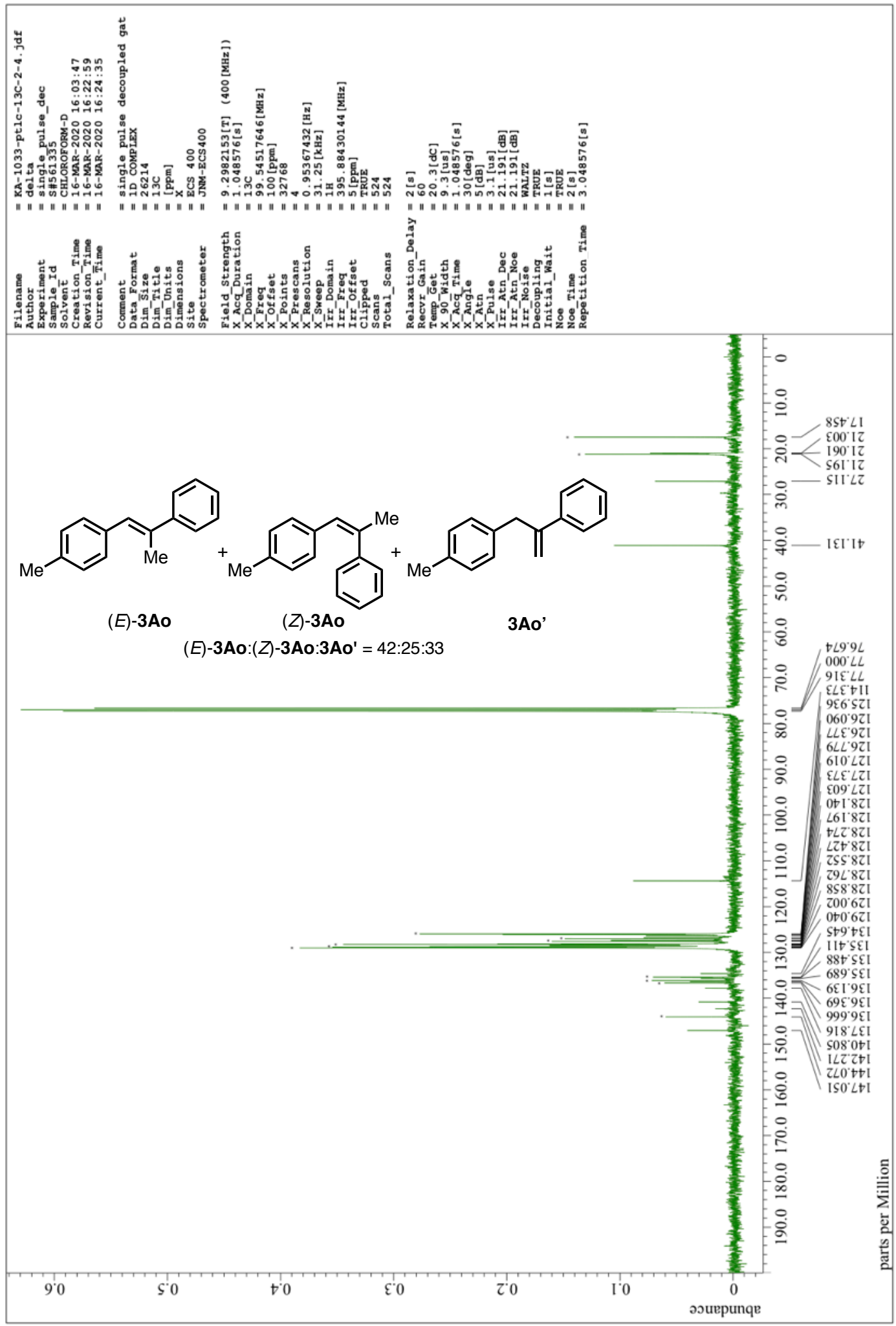


${ }^{1} \mathrm{H}$ NMR of $6\left(400 \mathrm{MHz}, \mathrm{CDCl}_{3}\right)$

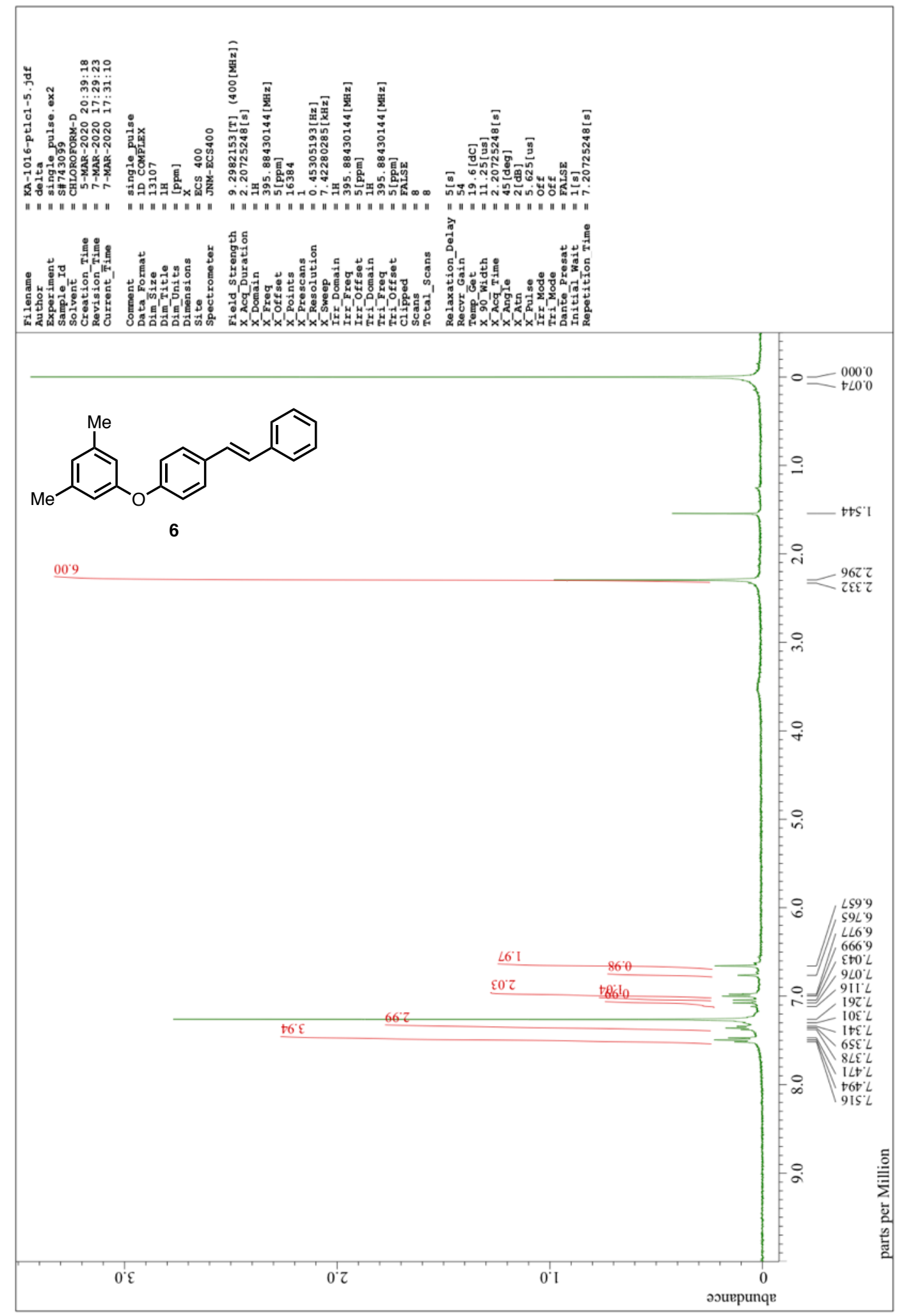


${ }^{13} \mathrm{C}$ NMR of $6\left(101 \mathrm{MHz}, \mathrm{CDCl}_{3}\right)$

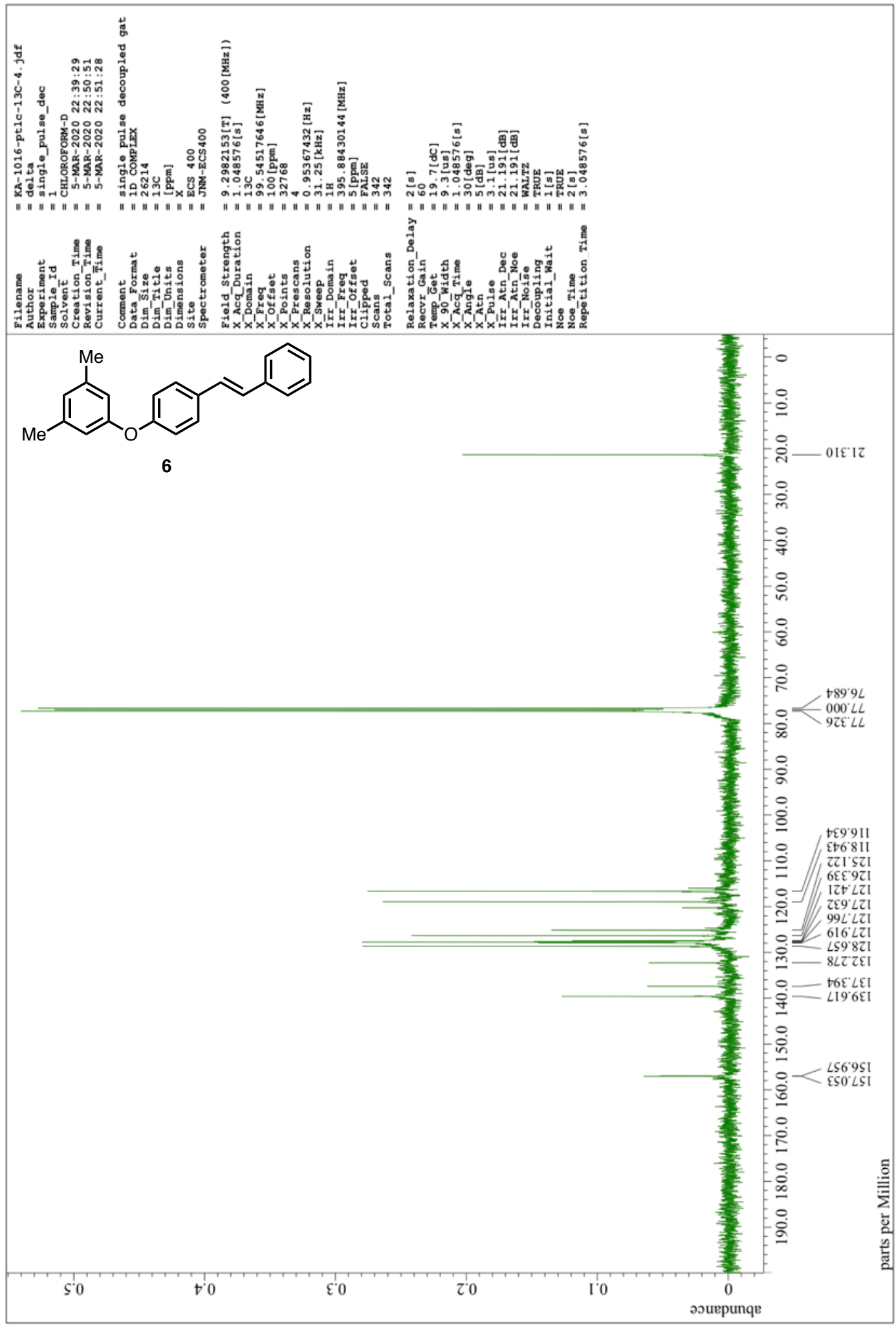

